Supporting Information

\title{
(+)-Sorangicin A Synthetic Studies. Construction of the C(1-15)
} and C(16-29) Subtargets

\author{
Amos B. Smith, III*, Richard J. Fox, and John A. Vanecko \\ Department of Chemistry, Laboratory for Research on the Structure of Matter, and Monell Chemical \\ Senses Center, University of Pennsylvania, Philadelphia, PA 19104, U.S.A.
}




\section{Materials and Methods}

Except as otherwise indicated, all reactions were carried out under an argon atmosphere in flame- or oven-dried glassware, and solvents were freshly distilled. The argon was deoxygenated and dried by passage through an OXICLEAR ${ }^{\mathrm{TM}}$ filter from Aldrich and Drierite tube, respectively. Diethyl either $\left(\mathrm{Et}_{2} \mathrm{O}\right)$ and tetrahydrofuran (THF) were distilled from sodium/benzophenone. Dichloromethane $\left(\mathrm{CH}_{2} \mathrm{Cl}_{2}\right)$, benzene and diisopropylamine were distilled from calcium hydride. Triethylamine and 2,6lutidine were distilled from calcium hydride and stored over potassium hydroxide. Anhydrous pyridine, dimethylformamide and dimethyl sulfoxide were purchased from Aldrich and used without purification. $n$ Butylithium ( $n$-BuLi) and $t$-butyllithium ( $t$-BuLi) were purchased from Aldrich and titrated against $n$ benzylbenzamide prior to use. ${ }^{1}$ Chlorotriethylsilane (TESCI), tert-butyldimethylsilyl trifluoromethanesulfonate (TBSOTf) and 2,4,6-collidine were distilled from calcium hydride. Acetone $(50 \mathrm{~mL})$ was stirred with $\mathrm{K}_{2} \mathrm{CO}_{3}(1.5 \mathrm{~g})$ overnight under argon, and distilled onto activated $4 \AA$ molecular sieves. All azeotroping was performed from benzene. Except as indicated otherwise, all other reagents were purchased from Aldrich, Acros, or Strem chemicals and used as received.

Reactions were magnetically stirred and monitored by thin layer chromatography (TLC) with 0.25 mm E. Merck pre-coated silica gel plates. Silica gel for flash chromatography (particle size 0.040-0.063 $\mathrm{mm}$ ) was supplied by Bodman, Silicycle and Sorbent Technologies. Yields refer to chromatographically and spectroscopically pure compounds, unless otherwise stated.

All melting points were determined on a Bristoline heated-stage microscope or Thomas-Hoover apparatus and are corrected. Infrared spectra were recorded on either a Perkin-Elmer Model 283B, Perkin-Elmer Model 1600 FTIR, or Jasco FTIR-480plus spectrometer. ${ }^{1} \mathrm{H}$ and ${ }^{13} \mathrm{C}$ spectra were recorded on a Bruker AMX-500 spectrometer. Chemical shifts are reported as $\delta$ values relative to internal chloroform ( $\delta 7.24$ or 7.26), benzene $(\delta 7.15)$ or DMSO $(\delta 2.49)$ for ${ }^{1} \mathrm{H}$ and either chloroform $(\delta 77.0)$, benzene $(\delta 128.0)$ or DMSO $(\delta 39.50)$ for ${ }^{13} \mathrm{C}$. Optical rotations were obtained with a Perkin-Elmer model 241 polarimeter in the solvent indicated. High resolution mass spectra were measured at the University of Pennsylvania Mass Spectrometry Center by Dr. Rakesh Kohli or Mr. John Dykins on either a VG Micromass $70 / 70 \mathrm{H}$ or VG ZAB-E spectrometer. 


\section{Experimental Procedures}

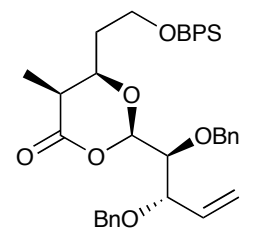

$(+)-10$

Dioxanone (+)-10. Aldehyde (+)-7 (0.37 g, 1.0 equiv.) and the known bis-TMS compound ${ }^{2}$ derived from (+)-6 (0.73 g, 1.1 equiv.) were combined in a flask and azeotroped with benzene ( $3 \times 5 \mathrm{~mL})$. The mixture was then dissolved in dichloromethane $(4 \mathrm{~mL})$ and cooled to $-78{ }^{\circ} \mathrm{C}$. In a separate flask were combined di-t-butylmethylpyridine (DTBMP, $104 \mathrm{mg})$, dichloromethane $(2 \mathrm{~mL})$, and TMSOTf $(0.4$ $\mathrm{mL})$ sequentially. Using a syringe, the pre-mixed TMSOTf/DTBMP solution $(0.75 \mathrm{~mL})$ was added dropwise to the reaction flask and the resulting solution was allowed to mix at $-78{ }^{\circ} \mathrm{C}$ for $11 \mathrm{~h}$ at which time it was then warmed to $-50^{\circ} \mathrm{C}$ for $15 \mathrm{~min}$. Upon cooling back to $-78{ }^{\circ} \mathrm{C}$, the reaction was quenched by the rapid addition of triethylamine ( $1.7 \mathrm{~mL}, 10$ equiv.) and following removal of the ice bath, allowed to mix for $20 \mathrm{~min}$. The contents of the flask were transferred to a separatory funnel containing dichloromethane $(50 \mathrm{~mL})$. The organics were washed with saturated sodium bicarbonate $(2 \times 20 \mathrm{~mL})$ and the combined aqueous layers then washed with dichloromethane $(30 \mathrm{~mL})$. The combined organics were then dried over $\mathrm{Na}_{2} \mathrm{SO}_{4}$ and concentrated to provide a yellow oil. Purification was accomplished using column chromatography on silylated silica gel (20\% EtOAc/Hexanes). The product containing fractions were then transferred to a separatory funnel and washed with a $3.6 \mathrm{M} \mathrm{NaHSO}_{3}$ solution $(30 \mathrm{~mL})$, dried over $\mathrm{Na}_{2} \mathrm{SO}_{4}$, and concentrated to yield dioxonone (+)-10 (0.83 $\mathrm{g}, 99 \%$ yield) as a yellow oil. The compound was immediately carried on to the next reaction: IR (neat) $2928,1750,1427,1226,1111 \mathrm{~cm}^{-1}$; $[\alpha]_{\mathrm{D}}^{20}+4.5\left(c\right.$ 1.10, $\left.\mathrm{CHCl}_{3}\right) ;{ }^{1} \mathrm{H}$ NMR $\left(500 \mathrm{MHz}, \mathrm{CDCl}_{3}\right) \delta$ 7.61-7.53 (m, $\left.3 \mathrm{H}\right)$, 7.37-7.19 (m, $\left.17 \mathrm{H}\right), 5.87$ (ddd, $J=17.7,10.3,7.7 \mathrm{~Hz}, 1 \mathrm{H}), 5.58(\mathrm{~d}, J=3.0 \mathrm{~Hz}, 1 \mathrm{H}), 5.38-5.31(\mathrm{~m}, 2 \mathrm{H}), 4.77\left(\mathrm{~d}, J_{A B}=11.4 \mathrm{~Hz}, 1\right.$ $\mathrm{H}), 4.67\left(\mathrm{~d}, \mathrm{~J}_{A B}=11.3 \mathrm{~Hz}, 1 \mathrm{H}\right), 4.61\left(\mathrm{~d}, J_{A B}=11.4 \mathrm{~Hz}, 1 \mathrm{H}\right), 4.39\left(\mathrm{~d}, J_{A B}=11.5 \mathrm{~Hz}, 1 \mathrm{H}\right), 4.27-4.21(\mathrm{~m}, 1$ H), $4.11(\mathrm{dd}, J=7.5,7.5 \mathrm{~Hz}, 1 \mathrm{H}), 3.79-3.74(\mathrm{~m}, 2 \mathrm{H}), 3.63(\mathrm{dd}, J=6.7,3.0 \mathrm{~Hz}, 1 \mathrm{H}), 2.71(\mathrm{~m}, 1 \mathrm{H}), 1.73$ (dddd, $J=12.5,6.2,6.2,6.2 \mathrm{~Hz}, 2 \mathrm{H}), 1.21$ (d, $J=7.3 \mathrm{~Hz}, 3 \mathrm{H}), 0.99(\mathrm{~s}, 9 \mathrm{H}) ;{ }^{13} \mathrm{C} \mathrm{NMR}\left(125 \mathrm{MHz}, \mathrm{CDCl}_{3}\right)$ $\delta 171.6,138.2,138.1,135.5,135.3,133.5,133.4,129.8,128.4,128.3,128.0,127.9,127.7,127.6,127.5$, 
119.6, 101.3, 80.5, 79.3, 75.0, 73.5, 70.6, 59.6, 39.5, 34.1, 30.1, 26.8, 11.9; high resolution mass spectrum (ES+) $m / z 687.3100\left[(\mathrm{M}+\mathrm{Na})^{+}\right.$; calcd for $\left.\mathrm{C}_{41} \mathrm{H}_{48} \mathrm{O}_{6} \mathrm{SiNa}: 687.3220\right]$.

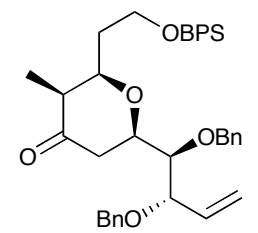

$(+)-5$

Tetrahydropyranone (+)-5. Dioxanone (+)-10 (0.83 g, 1.0 equiv.) was dissolved in a $0.7 \mathrm{M}$ solution in THF of Tebbe reagent $(14 \mathrm{~mL})$ and the resulting solution was then heated to $62{ }^{\circ} \mathrm{C}$ for $8 \mathrm{~h}$ or until the reaction was judged complete by thin layer chromatography (thin layer chromatography sample was taken from the reaction mixture and diluted with hexanes prior to spotting on chromatography plates). Upon completion, the reaction was cooled to $\mathrm{rt}$ and $25 \mathrm{~mL}$ of hexanes was added and the resulting solution mixed for $20 \mathrm{~min}$. Filtration through a pad of celite was then initiated followed by washing the celite pad with $50 \mathrm{~mL}$ of hexanes. Concentration of the filtrate was followed by first being passed through a plug of silylated silica gel using $10 \%$ EtOAc/Hexanes. The product containing fractions were concentrated and once again passed through a second plug of silylated silica using $10 \% \mathrm{EtOAc/Hexanes.}$ The filtrate was concentrated to yield the crude enol ether $(0.66 \mathrm{~g}, 80 \%$ yield) as a yellow oil which was carried as is into the rearrangement. The crude enol ether $(0.66 \mathrm{~g}, 0.996 \mathrm{mmol}, 1.0$ equiv.) was dissolved in dichloromethane $(20 \mathrm{~mL})$ and cooled to $-78{ }^{\circ} \mathrm{C}$. AlMe ${ }_{2} \mathrm{Cl}(1.5 \mathrm{~mL}, 1.5$ equiv.) was then added dropwise and the resulting mixture stirred for $40 \mathrm{~min}$. At this point the bath was removed and the mixture allowed to warm for $20 \mathrm{~min}$ and then re-cooled to $-78{ }^{\circ} \mathrm{C}$ and quenched by rapid addition of $3 \mathrm{~mL}$ of sat. $\mathrm{NaHCO}_{3}$. Contents were warmed to $\mathrm{rt}$ and then poured into a separatory funnel containing $25 \mathrm{~mL}$ of $\mathrm{NaHCO}_{3}$ and $25 \mathrm{~mL}$ of dichloromethane. The organic layer was separated and the aqueous washed with $2 \times 20 \mathrm{~mL}$ of dichloromethane. The collected organics were then dried $\left(\mathrm{MgSO}_{4}\right)$ and concentrated to provide an oil. Column chromatography (10\% EtOAc/Hexanes) provided tetrahydropyranone (+)-5 as a colorless oil $\left(0.462 \mathrm{~g}, 70 \%\right.$ yield): IR (neat) $2929,2857,1779,1458,1389,1359,1215,1111 \mathrm{~cm}^{-1} ;[\alpha]_{D}^{20}$ +37.3 $\left(c\right.$ 1.65, $\left.\mathrm{CHCl}_{3}\right) ;{ }^{1} \mathrm{H}$ NMR $\left(500 \mathrm{MHz}, \mathrm{CDCl}_{3}\right) \delta$ 7.64-7.60 (m, 3 H), 7.40-7.20 (m, $\left.17 \mathrm{H}\right), 5.80(\mathrm{ddd}, J=$ 17.5, 10.4, 7.8 Hz, $1 \mathrm{H}), 5.32-5.20(\mathrm{~m}, 2 \mathrm{H}), 4.69(\operatorname{app~s}, 2 \mathrm{H}), 4.57\left(\mathrm{~d}, \mathrm{~J}_{\mathrm{AB}}=11.9 \mathrm{~Hz}, 1 \mathrm{H}\right), 4.26\left(\mathrm{~d}, \mathrm{~J}_{\mathrm{AB}}=\right.$ 
$11.9 \mathrm{~Hz}, 1 \mathrm{H}), 3.84-3.68(\mathrm{~m}, 6 \mathrm{H}), 2.61(\mathrm{dd}, J=14.5,11.5 \mathrm{~Hz}, 1 \mathrm{H}), 2.30-2.16(\mathrm{~m}, 2 \mathrm{H}), 1.85-1.76(\mathrm{~m}, 1$ H), 1.62-1.52 (m, $1 \mathrm{H}), 1.04(\mathrm{~d}, J=7.4 \mathrm{~Hz}, 3 \mathrm{H}), 1.01$ (s, $9 \mathrm{H}) ;{ }^{13} \mathrm{C}$ NMR $\left(125 \mathrm{MHz}, \mathrm{CDCl}_{3}\right) \delta$ 212.0, 138.4, $138.1,135.5,135.4,133.7,129.7,128.4,128.3,128.2,127.7,127.6,119.7,82.4,80.3,75.5,74.5,70.4$, $60.5,49.2,38.9,35.0,29.7,26.8,19.2,10.9$ : high resolution mass spectrum (ES+) $\mathrm{m} / \mathrm{z} 685.3307$ $\left[(\mathrm{M}+\mathrm{Na})^{+}\right.$; calcd for $\left.\mathrm{C}_{42} \mathrm{H}_{50} \mathrm{O}_{5} \mathrm{SiNa}: 685.3428\right]$.

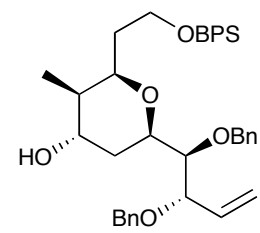

$(+)-11 \mathrm{a}$

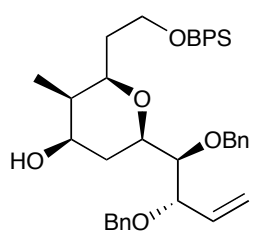

$(+)-11 b$

DIBAL Reduction. To a solution of $(+)-5(0.11 \mathrm{~g}, 0.305 \mathrm{mmol}, 1.0$ equiv. $)$ in dichloromethane (3.1 mL) cooled to $-78{ }^{\circ} \mathrm{C}$ was added DIBAL (0.61 mL, 2.0 equiv., $1.0 \mathrm{M}$ solution in hexanes). The reaction was maintained at $-78{ }^{\circ} \mathrm{C}$ until complete consumption of starting material as judged by TLC analysis. The reaction was quenched by the addition of a $1: 2$ mixture of celite to $\mathrm{NaSO}_{4} \cdot 10 \mathrm{H}_{2} \mathrm{O}$ and then allowed to warm to rt. Filtration and washing of the filter cake with $2 \times 50 \mathrm{~mL}$ dichloromethane was followed by concentration to yield a $1.0: 1.1$ mixture of alcohols (by NMR) favoring the undesired equatorial alcohol as an oil. This mixture was separated using careful column chromatography (10\% EtOAc/ Hexanes) to provide (+)-11a (desired) (0.093 g, 46\% yield) and (+)-11b (undesired) $(0.104 \mathrm{~g}, 50 \%$ yield). For 11a: IR (neat) 3030, 2927, 1916, 1507, 1465, 1344, 1324, 1208, 1110, 1070, $1028 \mathrm{~cm}^{-1} ;[\alpha]_{D}^{20}$ +29.0 (c 1.80, $\left.\mathrm{CHCl}_{3}\right) ;{ }^{1} \mathrm{H}$ NMR $\left(500 \mathrm{MHz}, \mathrm{CDCl}_{3}\right) \delta$ 7.70-7.59 (m, 3 H), 7.42-7.18 (m, $\left.17 \mathrm{H}\right), 5.84$ (ddd, $J=$ 17.9, 10.1, 7.8 Hz, $1 \mathrm{H}), 5.28-5.14(\mathrm{~m}, 2 \mathrm{H}), 4.74\left(\mathrm{~d}, \mathrm{~J}_{\mathrm{AB}}=11.2 \mathrm{~Hz}, 1 \mathrm{H}\right), 4.62\left(\mathrm{~d}, \mathrm{~J}_{\mathrm{AB}}=11.2 \mathrm{~Hz}, 1 \mathrm{H}\right), 4.56$ $\left(\mathrm{d}, J_{\mathrm{AB}}=11.5 \mathrm{~Hz}, 1 \mathrm{H}\right), 4.26\left(\mathrm{~d}, J_{\mathrm{AB}}=11.5 \mathrm{~Hz}, 1 \mathrm{H}\right), 3.93(\mathrm{dd}, J=7.8,4.5 \mathrm{~Hz}, 1 \mathrm{H}), 3.80-3.66(\mathrm{~m}, 3 \mathrm{H})$, $3.60(\mathrm{dd}, J=6.0,4.5 \mathrm{~Hz}, 1 \mathrm{H}), 3.47-3.42(\mathrm{~m}, 1 \mathrm{H}), 3.41-3.34(\mathrm{~m}, 1 \mathrm{H}), 1.82-1.63(\mathrm{~m}, 5 \mathrm{H}), 1.03(\mathrm{~s}, 9 \mathrm{H})$, $0.80(\mathrm{~d}, J=6.7 \mathrm{~Hz}, 3 \mathrm{H}) ;{ }^{13} \mathrm{C} \mathrm{NMR}\left(125 \mathrm{MHz}, \mathrm{CDCl}_{3}\right) \delta 138.8,138.7,135.5,135.3,134.0,129.6,128.3$, 128.2, 127.7, 127.6, 127.5, 127.4, 119.3, 82.8, 81.1, 75.8, 75.4, 74.5, 71.3, 70.4, 61.1, 38.4, 36.0, 30.5, 26.9, 19.2, 5.0; high resolution mass spectrum (ES+) $\mathrm{m} / \mathrm{z} 687.3488\left[(\mathrm{M}+\mathrm{Na})^{+}\right.$; calcd for $\mathrm{C}_{42} \mathrm{H}_{52} \mathrm{O}_{5} \mathrm{SiNa}$ : 687.3584]. For 11b: IR (neat) 3030, 2980, 1959, 1496, 1465, 1362, 1330, 1208, 1110, 1070, $1028 \mathrm{~cm}^{-1}$; $[\alpha]_{\mathrm{D}}^{20}+16.0\left(c \mathrm{0} .95, \mathrm{CHCl}_{3}\right) ;{ }^{1} \mathrm{H}$ NMR $\left(500 \mathrm{MHz}, \mathrm{CDCl}_{3}\right) \delta$ 7.65-7.60 (m, $\left.4 \mathrm{H}\right), 7.40-7.20(\mathrm{~m}, 16 \mathrm{H}), 5.83$ 
(ddd, $J=17.5,10.4,7.8 \mathrm{~Hz}, 1 \mathrm{H}), 5.26-5.10(\mathrm{~m}, 2 \mathrm{H}), 4.73\left(\mathrm{~d}, \mathrm{~J}_{\mathrm{AB}}=11.5 \mathrm{~Hz}, 1 \mathrm{H}\right), 4.62\left(\mathrm{~d}, \mathrm{~J}_{\mathrm{AB}}=11.5 \mathrm{~Hz}\right.$, $1 \mathrm{H}), 4.53\left(\mathrm{~d}, J_{\mathrm{AB}}=11.9 \mathrm{~Hz}, 1 \mathrm{H}\right), 4.24\left(\mathrm{~d}, J_{\mathrm{AB}}=11.9 \mathrm{~Hz}, 1 \mathrm{H}\right), 3.95-3.82(\mathrm{~m}, 3 \mathrm{H}), 3.79-3.66(\mathrm{~m}, 4 \mathrm{H})$, 3.54 (dd, $J=6.0,4.5 \mathrm{~Hz}, 1 \mathrm{H}), 1.80-1.63(\mathrm{~m}, 2 \mathrm{H}), 1.57-1.42(\mathrm{~m}, 3 \mathrm{H}), 1.03$ (s, $9 \mathrm{H}), 0.84$ (d, J = 7.1 Hz, 3 $\mathrm{H}) ;{ }^{13} \mathrm{C}$ NMR $\left(125 \mathrm{MHz}, \mathrm{CDCl}_{3}\right) \delta 138.9,138.8,135.6,135.5,135.3,134.1,134.0,129.5,128.3,128.2$, $128.1,127.7,127.6,127.4,119.3,83.2,81.1,74.4,72.0,70.9,70.6,70.4,61.5,38.3,36.0,29.7,26.9$, 19.2, 11.1; high resolution mass spectrum (ES+) $\mathrm{m} / \mathrm{z} 687.3483\left[(\mathrm{M}+\mathrm{Na})^{+}\right.$; calcd for $\mathrm{C}_{42} \mathrm{H}_{52} \mathrm{O}_{5} \mathrm{SiNa}$ : 687.3584].

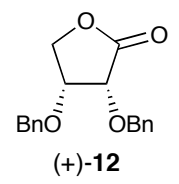

Lactone (+)-12. To a solution of D-erythronolactone $(10.0 \mathrm{~g}, 84.7 \mathrm{mmol}, 1.0$ equiv.) in dry acetonitrile $(500 \mathrm{~mL})$ at room temperature was added benzyl bromide $(75.0 \mathrm{~mL})$. Anhydrous calcium sulfate (59.1 g, 5.0 equiv.) was then added and the resulting mixture was stirred for $5 \mathrm{~min}$ at which time silver (I) oxide (39.2 g, 2.0 equiv.) was added in three portions over $5 \mathrm{~min}$. The resulting reaction flask was covered in aluminum foil and allowed to mix vigorously at room temperature for $12 \mathrm{~h}$ at which point a second portion of silver (I) oxide (39.2 g, 2.0 equiv.) was added and the resulting mixture allowed to mix for $36 \mathrm{~h}$. The reaction mixture was then filtered through a plug of celite to remove the solids, and the resulting filter cake was washed with $3 \times 250 \mathrm{~mL}$ of acetonitrile. Concentration of the combined organics was followed by column chromatography (30\% EtOAc/Hexanes to $70 \%$ EtOAc/Hexanes to $100 \%$ EtOAc) to yield $(+)-12(22.9 \mathrm{~g}, 91 \%$ yield $)$. The experimental data agreed with that present in the literature (Marshall, J. A.; Seletsky, B. M.; Luke, G. P. J. Org. Chem. 1994, 59, 3413-3420).

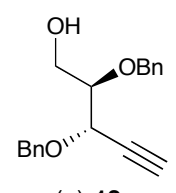

$(+)-13$

Alkyne (+)-13. To a solution of (+)-12 (8.90 g, $29.9 \mathrm{mmol}, 1.0$ equiv.) in dichloromethane (300 $\mathrm{mL}$ ) cooled to $-78{ }^{\circ} \mathrm{C}$ was added DIBAL (39 mL, 1.3 equiv., $1.0 \mathrm{M}$ solution in hexanes). The resulting solution was mixed at $-78{ }^{\circ} \mathrm{C}$ for $1 \mathrm{~h}$ at which time it was quenched using a 2:1 mixture of $\mathrm{Na}_{2} \mathrm{SO}_{4} \cdot 10 \mathrm{H}_{2} \mathrm{O}$ SI6 
/ Celite until mixing became difficult. At this time the cooling bath was removed and enough dichloromethane was added to afford a vigorously stirring solution. Stirring was then continued for $10 \mathrm{~h}$ at which time the solution was filtered and the filter cake was washed with $2 \times 500 \mathrm{~mL}$ of ethyl acetate. Concentration of the organics afforded the crude lactol as an oil $(8.77 \mathrm{~g}, 98 \%$ yield) which was used without further purification. To a flask containing THF (300 mL) was added a $2.0 \mathrm{M}$ solution in hexanes of LDA (38 mL, 2.6 equiv.). The resulting solution was cooled to $-78{ }^{\circ} \mathrm{C}$ at which time a solution of trimethylsilyldiazomethane (19 mL, 1.3 equiv., $2.0 \mathrm{M}$ in ether) was added dropwise and the reaction stirred for $30 \mathrm{~min}$. A solution of the crude lactol $(8.8 \mathrm{~g}, 29 \mathrm{mmol}, 1.0$ equiv.) in THF $(25 \mathrm{~mL})$ was then added dropwise to the reaction flask, the bath was removed, and the reaction allowed to warm to rt. Upon warming the release of $\mathrm{N}_{2}$ can be observed and care should be taken to have adequate ventilation. The reaction is monitored by TLC, and upon consumption of starting material, is quenched by the addition of sat. $\mathrm{NH}_{4} \mathrm{Cl}(50 \mathrm{~mL})$. Separation of the organic phase is followed by washing of the aqueous phase with $2 \times 50 \mathrm{~mL}$ ether. The combined organics were dried $\left(\mathrm{MgSO}_{4}\right)$ and concentrated. The resulting yellow oil is purified by column chromatography eluting with $20 \%$ EtOAc/Hexanes. The product containing fractions were collected and concentrated to yield (+)-13 (7.4 g, 87\% yield) as a yellow oil: IR (neat) 3296, 3030, 2916, 1495, 1454, $1089 \mathrm{~cm}^{-1} ;[\alpha]_{\mathrm{D}}{ }^{20}+93.5\left(c 0.97, \mathrm{CHCl}_{3}\right) ;{ }^{1} \mathrm{H}$ NMR $\left(500 \mathrm{MHz}, \mathrm{CDCl}_{3}\right) \delta$ 7.43-7.28 (m, 10 $\mathrm{H}), 4.86\left(\mathrm{~d}, \mathrm{~J}_{\mathrm{AB}}=11.7 \mathrm{~Hz}, 1 \mathrm{H}\right), 4.81\left(\mathrm{~d}, J_{\mathrm{AB}}=11.7 \mathrm{~Hz}, 1 \mathrm{H}\right), 4.65\left(\mathrm{~d}, J_{\mathrm{AB}}=11.7 \mathrm{~Hz}, 1 \mathrm{H}\right), 4.55\left(\mathrm{~d}, J_{\mathrm{AB}}=\right.$ $11.7 \mathrm{~Hz}, 1 \mathrm{H}), 4.31(\mathrm{dd}, J=5.3,2.2 \mathrm{~Hz}, 1 \mathrm{H}), 3.81-3.78(\mathrm{~m}, 2 \mathrm{H}), 3.72$ (ddd, $J=5.3,5.3,4.7 \mathrm{~Hz}, 1 \mathrm{H})$, $2.56(\mathrm{~d}, J=2.2 \mathrm{~Hz}, 1 \mathrm{H}), 2.09(\mathrm{br} \mathrm{s}, 1 \mathrm{H}) ;{ }^{13} \mathrm{C} \mathrm{NMR}\left(125 \mathrm{MHz}, \mathrm{CDCl}_{3}\right) \delta$ 141.5, 140.7, 131.6, 131.3, 131.0, $130.9,130.6,129.9,80.9,80.7,75.4,72.9,70.6,68.6,61.0$; high resolution mass spectrum (ES+) $\mathrm{m} / \mathrm{z}$ $319.1317\left[(\mathrm{M}+\mathrm{Na})^{+}\right.$; calcd for $\left.\mathrm{C}_{19} \mathrm{H}_{20} \mathrm{O}_{3} \mathrm{Na}: 319.1412\right]$.

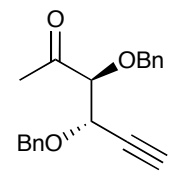

$(+)-14$

Ketone (+)-14. To a flask containing alcohol (+)-13 (6.5 g, $22 \mathrm{mmol}, 1.0$ equiv.) was added dichloromethane $(220 \mathrm{~mL})$. Dimethylsulfoxide $(50 \mathrm{~mL})$, triethylamine $\left(18 \mathrm{~mL}, 6.0\right.$ equiv.), and $\mathrm{SO}_{3} \cdot P y r$ (10.4 g, 3.0 equiv.) were then added sequentially. The reaction was then stirred at $\mathrm{rt}$ until judged 
complete by TLC. At this point the dichloromethane was removed under vacuum and the resulting syrup was taken up in $600 \mathrm{~mL}$ of pentane. The organic layer was then washed with $3 \times 150 \mathrm{~mL}$ of a sat. $\mathrm{CuSO}_{4}$ solution followed by $150 \mathrm{~mL}$ of water. The organic phase was then dried $\left(\mathrm{MgSO}_{4}\right)$ and concentrated to yield a pale yellow oil ( $6.3 \mathrm{~g}, 98 \%$ yield) of the desired aldehyde that was used without further purification. To a flask containing the crude aldehyde $(6.3 \mathrm{~g}, 21 \mathrm{mmol}, 1.0$ equiv.) was added dry toluene and the resulting solution was cooled to $-10{ }^{\circ} \mathrm{C} . \mathrm{AlMe}_{3}$ (38 mL, 3.5 equiv.) was then added dropwise. The resulting solution was allowed to mix until TLC judged the reaction complete. Quenching was accomplished by careful addition of a $2: 1$ mixture of a $\mathrm{NaSO}_{4} \cdot 10 \mathrm{H}_{2} \mathrm{O}$ :celite mixture and vigorously stirred at $\mathrm{rt}$ for $4 \mathrm{~h}$. Filtration and washing of the filter cake with $2 \times 300 \mathrm{~mL}$ of EtOAc was followed by concentration of the organics to yield the crude alcohol $(5.3 \mathrm{~g}, 78 \%$ yield) which was carried on to the next step without further purification. The same oxidation and workup as for $(+)-13$ was performed using the following amounts. The alcohol (5.3 g, $17 \mathrm{mmol}, 1.0$ equiv.) was dissolved in dichloromethane (170 mL). Dimethylsulfoxide $(50 \mathrm{~mL})$, triethylamine $\left(14 \mathrm{~mL}, 6.0\right.$ equiv.), and $\mathrm{SO}_{3} \cdot \mathrm{Pyr}(7.9 \mathrm{~g}, 3.0$ equiv.) were added sequentially and the reaction mixed until judged complete by TLC. The resulting ketone (+)-14 was isolated as a yellow oil $(4.9 \mathrm{~g}, 97 \%$ yield) after purification by column chromatography $(20 \%$ EtOAc/Hexanes): IR (neat) 3283, 3031, 2869, 1720, 1496, 1454, 1354, 1208, $1092 \mathrm{~cm}^{-1} ;[\alpha]_{\mathrm{D}}^{20}+72.2(c$ 1.13, $\left.\mathrm{CHCl}_{3}\right) ;{ }^{1} \mathrm{H}$ NMR $\left(500 \mathrm{MHz}, \mathrm{CDCl}_{3}\right) \delta 7.38-7.26(\mathrm{~m}, 10 \mathrm{H}), 4.82\left(\mathrm{~d}, \mathrm{~J}_{\mathrm{AB}}=11.9 \mathrm{~Hz}, 1 \mathrm{H}\right), 4.74\left(\mathrm{~d}, J_{\mathrm{AB}}=\right.$ $11.9 \mathrm{~Hz}, 1 \mathrm{H}), 4.68\left(\mathrm{~d}, J_{\mathrm{AB}}=11.9 \mathrm{~Hz}, 1 \mathrm{H}\right), 4.53\left(\mathrm{~d}, \mathrm{~J}_{\mathrm{AB}}=11.9 \mathrm{~Hz}, 1 \mathrm{H}\right), 4.46(\mathrm{dd}, J=4.8,2.2 \mathrm{~Hz}, 1 \mathrm{H})$, $4.05(\mathrm{~d}, J=4.8 \mathrm{~Hz}, 1 \mathrm{H}), 2.58(\mathrm{~d}, J=2.2 \mathrm{~Hz}, 1 \mathrm{H}), 2.19(\mathrm{~s}, 3 \mathrm{H}) ;{ }^{13} \mathrm{C}$ NMR $\left(125 \mathrm{MHz}, \mathrm{CDCl}_{3}\right) \delta 137.1$, $136.9,128.4,128.0,127.9,85.5,79.1,76.7,76.1,73.4,70.9,69.7,27.2 ;$ high resolution mass spectrum (ES+) $m / z 331.1324\left[(\mathrm{M}+\mathrm{Na})^{+} ;\right.$calcd for $\left.\mathrm{C}_{20} \mathrm{H}_{20} \mathrm{O}_{3} \mathrm{Na}: 331.1412\right]$.

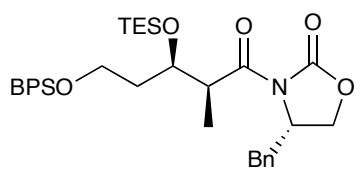

$(+)-$ SI1

TES ether (+)-SI1. To a flask containing (+)-15 (10.1 g, 18.5 mmol, 1.0 equiv.) was added a 1:3 solution of THF/DMF (190 mL). Imidazole (3.2 g, 2.5 equiv.) was then added followed by TESCl $(4.0 \mathrm{~mL}$, 1.3 equiv.) and the resulting mixture allowed to stir overnight at rt. Concentration and column 
chromatography of the crude reaction mixture (20\% EtOAc/Hexanes) provided the desired TES protected alcohol (+)-SI1 (11.6 g, 95\% yield) as a colorless oil: IR (neat) 2955, 2876, 1783, 1701, 1457, 1427, 1382, 1348, 1236, 1208, $1111 \mathrm{~cm}^{-1} ;[\alpha]_{\mathrm{D}}{ }^{20}+30.9\left(c 0.45, \mathrm{CHCl}_{3}\right) ;{ }^{1} \mathrm{H}$ NMR $\left(500 \mathrm{MHz}, \mathrm{CDCl}_{3}\right) \delta 7.73-7.66$ (m, $3 \mathrm{H}), 7.45-7.20(\mathrm{~m}, 12 \mathrm{H}), 4.58$ (dddd, $J=9.6,7.4,2.9,2.2 \mathrm{~Hz}, 1 \mathrm{H}), 4.20$ (ddd, $J=7.0,4.8,4.8 \mathrm{~Hz}, 1$ H), 4.15 (dd, $J=9.3,2.2 \mathrm{~Hz}, 1 \mathrm{H}$ ), 4.07 (dd, $J=9.3,7.0 \mathrm{~Hz}, 1 \mathrm{H}$ ), 3.93 (dddd, $J=6.7,6.7,6.7,5.2 \mathrm{~Hz}, 1$ H), 3.78-3.73 (m, $2 \mathrm{H}), 3.29(\mathrm{dd}, J=13.4,3.3 \mathrm{~Hz}, 1 \mathrm{H}), 2.78(\mathrm{dd}, J=13.4,9.6 \mathrm{~Hz}, 1 \mathrm{H}), 1.92-1.85(\mathrm{~m}, 1$ H), 1.82-1.74 (m, $1 \mathrm{H}), 1.22(\mathrm{~d}, J=6.7 \mathrm{~Hz}, 3 \mathrm{H}), 1.07(\mathrm{~s}, 9 \mathrm{H}), 0.92(\mathrm{t}, J=7.8 \mathrm{~Hz}, 9 \mathrm{H}), 0.57$ (q, J = 7.8 $\mathrm{Hz}, 6 \mathrm{H}) ;{ }^{13} \mathrm{C} \mathrm{NMR}\left(125 \mathrm{MHz}, \mathrm{CDCl}_{3}\right) \delta 175.1,152.9,135.6,135.5,133.9,129.5,129.4,128.9,128.3$, $127.7,127.6,127.3,70.3,65.9,60.5,55.7,43.4,38.1,37.7,26.8,19.1,12.3,6.9,5.0$; high resolution mass spectrum (ES+) $\mathrm{m} / \mathrm{z} 682.3385\left[(\mathrm{M}+\mathrm{Na})^{+}\right.$; calcd for $\left.\mathrm{C}_{38} \mathrm{H}_{53} \mathrm{NO}_{5} \mathrm{Si}_{2} \mathrm{Na}: 682.3462\right]$.

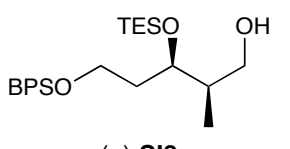

$(+)-\mathrm{SI} 2$

Alcohol (+)-SI2. To a flask containing (+)-SI1 (11.2 g, $17.0 \mathrm{mmol}, 1.0$ equiv.) was added ether $(60 \mathrm{~mL})$ followed by water $(0.4 \mathrm{~mL})$. This solution was then cooled to $0{ }^{\circ} \mathrm{C}$ and a $2.0 \mathrm{M} \mathrm{THF}$ solution of $\mathrm{LiBH}_{4}$ (10.2 mL, 1.2 equiv.) was then added dropwise. The solution became cloudy white. The ice bath was then removed and the solution warmed to rt. Upon complete consumption of starting material as judged by TLC, the reaction was cooled back to $0{ }^{\circ} \mathrm{C}$, and quenched by addition of a sat. $\mathrm{NaHCO}_{3}$ solution $(50 \mathrm{~mL})$. This mixture was allowed to stir for $20 \mathrm{~min}$ before separating the layers. The aqueous layer was then washed with $2 \times 100 \mathrm{~mL}$ of ether before washing the combined organics with $100 \mathrm{~mL}$ of brine. Drying of the organics $\left(\mathrm{MgSO}_{4}\right)$ was followed by concentration and column chromatography $(10 \%$ EtOAc/Hexanes) to provide (+)-SI2 (8.1 g, 98\% yield) as a colorless oil: IR (neat) 3448, 2955, 2875, 1785, $1701,1458,1427,1388,1238,1111,1007 \mathrm{~cm}^{-1} ;[\alpha]_{D}{ }^{20}+0.67\left(c 0.30, \mathrm{CHCl}_{3}\right) ;{ }^{1} \mathrm{H} \mathrm{NMR}\left(500 \mathrm{MHz}, \mathrm{CDCl}_{3}\right) \delta$ 7.70-7.64 (m, 4 H), 7.46-7.35 (m, $6 \mathrm{H}), 4.07$ (ddd, $J=7.8,4.8,3.3 \mathrm{~Hz}, 1 \mathrm{H}), 3.74(\mathrm{dd}, J=6.3,6.3 \mathrm{~Hz}, 2$ H), $3.67(\mathrm{dd}, J=9.6,9.6 \mathrm{~Hz}, 1 \mathrm{H}), 3.56-3.50(\mathrm{~m}, 1 \mathrm{H}), 2.74(\mathrm{br} \mathrm{s}, 1 \mathrm{H}), 1.99-1.91(\mathrm{~m}, 1 \mathrm{H})$, 1.76-1.67 (m, 2 H), $1.07(\mathrm{~s}, 9 \mathrm{H}), 0.94(\mathrm{t}, J=7.8 \mathrm{~Hz}, 9 \mathrm{H}), 0.81(\mathrm{~d}, J=7.0 \mathrm{~Hz}, 3 \mathrm{H}), 0.64-0.57(\mathrm{~m}, 6 \mathrm{H}) ;{ }^{13} \mathrm{C}$ NMR $(125$ $\left.\mathrm{MHz}, \mathrm{CDCl}_{3}\right) \delta 135.5,135.1,133.8,129.6,128.3,127.6,127.3,72.7,66.0,60.1,39.7,35.1,26.8,19.1$, 
12.1, 6.8, 5.0; high resolution mass spectrum (ES+) $\mathrm{m} / z$ 509.2985 $\left[(\mathrm{M}+\mathrm{Na})^{+}\right.$; calcd for $\mathrm{C}_{28} \mathrm{H}_{46} \mathrm{O}_{3} \mathrm{Si}_{2} \mathrm{Na}$ : 509.2985].

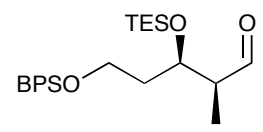

$(+)-16$

Aldehyde (+)-16. To a flask containing alcohol (+)-SI2 (1.71 g, $3.52 \mathrm{mmol}, 1.0$ equiv.) was added dichloromethane $(35 \mathrm{~mL})$ followed by dimethylsulfoxide $(3.5 \mathrm{~mL})$. To this solution was then added Hünigs' base (2.0 mL, 3.2 equiv.) followed by $\mathrm{SO}_{3} \cdot \mathrm{Pyr}(1.68 \mathrm{~g}, 3.0$ equiv.). The resulting mixture was stirred at $\mathrm{rt}$ until the reaction was judged complete by TLC. Work-up was then performed as described previously to yield (+)-16 as a yellow oil (1.67 g, 98\% yield): IR (neat) $3071,2955,2876,1707,1460,1427,1389$, 1237, $1111 \mathrm{~cm}^{-1} ;[\alpha]_{D}^{20}+8.70\left(c 0.92, \mathrm{CHCl}_{3}\right) ;{ }^{1} \mathrm{H}$ NMR $\left(500 \mathrm{MHz}, \mathrm{CDCl}_{3}\right) \delta 9.75(\mathrm{~s}, 1 \mathrm{H}), 7.68-7.64(\mathrm{~m}, 4$ H), 7.46-7.36 (m, $6 \mathrm{H}), 4.41$ (ddd, $J=6.7,6.7,3.3 \mathrm{~Hz}, 1 \mathrm{H}), 3.77-3.64(\mathrm{~m}, 2 \mathrm{H}), 2.44$ (dddd, $J=6.7,6.7$, 6.7, 3.3 Hz, $1 \mathrm{H}), 1.77-1.67(\mathrm{~m}, 2 \mathrm{H}), 1.06(\mathrm{~s}, 9 \mathrm{H}), 1.02(\mathrm{~d}, J=6.7 \mathrm{~Hz}, 3 \mathrm{H}), 0.92(\mathrm{t}, J=8.1 \mathrm{~Hz}, 9 \mathrm{H}), 0.57$ (q, $J=7.8 \mathrm{~Hz}, 6 \mathrm{H}) ;{ }^{13} \mathrm{C}$ NMR $\left(125 \mathrm{MHz}, \mathrm{CDCl}_{3}\right) \delta 205.1,135.5,133.5,129.7,129.6,127.6,127.5,69.0$, $60.4,51.3,37.2,26.8,19.1,7.6,6.8,5.0$. Attempts at obtaining high resolution mass spectral data under either electrospray or chemical ionization were unsuccessfull.

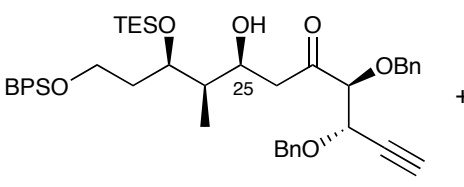

$(+)-17 a$

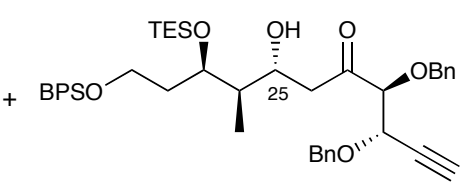

$(+)-17 b$

Alcohols (+)-17a and (+)-17b. To a flame-dried flask was added ether (8 $\mathrm{mL})$ followed by dicyclohexylboron chloride (1.1 mL, 1.1 equiv., 1.0 M in hexanes) and the resulting solution was cooled to $0{ }^{\circ} \mathrm{C}$. Triethylamine $(0.15 \mathrm{~mL}, 1.1$ equiv.) was then added and the solution became cloudy. This was mixed for $20 \mathrm{~min}$ and cooled to $-78{ }^{\circ} \mathrm{C}$ at which time a solution of ketone (+)-14 (0.31 g, $1.0 \mathrm{mmol}, 1.0$ equiv.) in ether $(2 \mathrm{~mL})$ was added dropwise. The resulting solution was warmed to $0{ }^{\circ} \mathrm{C}$ and allowed to mix at $0{ }^{\circ} \mathrm{C}$ for $1.5 \mathrm{~h}$ before being recooled to $-78^{\circ} \mathrm{C}$. A solution of aldehyde $(+)-16(0.58 \mathrm{~g}, 1.2$ equiv. $)$ in ether $(2 \mathrm{~mL})$ was then added dropwise and the resulting solution was mixed for $1 \mathrm{~h}$ and then warmed to $45^{\circ} \mathrm{C}$ and quenched with a $1: 1 \mathrm{MeOH} / \mathrm{pH} 7$ buffer solution $(50 \mathrm{~mL})$, keeping the temperature below -35 
${ }^{\circ} \mathrm{C}$. The contents were then poured into a flask containing $50 \mathrm{~mL}$ of ether at $0{ }^{\circ} \mathrm{C}$. To the resulting mixture was slowly added a $1: 1$ solution of $30 \% \mathrm{H}_{2} \mathrm{O}_{2} / \mathrm{pH} 7$ buffer $(50 \mathrm{~mL})$ making sure to keep the temperature below $5^{\circ} \mathrm{C}$. Upon complete addition, the resulting solution was mixed a further $1.5 \mathrm{~h}$ at $0{ }^{\circ} \mathrm{C}$. The layers were separated and the resulting aqueous phase was washed with $3 \times 50 \mathrm{~mL}$ ether. The combined organics were then dried with $\mathrm{MgSO}_{4}$ and concentrated to provide a crude oil. Purification was accomplished using column chromatography (5\% EtOAc/Hexanes to 10\% EtOAc/Hexanes) to provide (+)-17a (0.55 g, 72\% yield) and (+)-17b (0.18 g, 21\% yield) as clear, colorless oils. For 17a: IR (neat) $3448,3287,3096,2955,2876,1724,1471,1389,1240,1111 \mathrm{~cm}^{-1} ;[\alpha]_{D}{ }^{20}+47.3\left(c 1.24, \mathrm{CHCl}_{3}\right) ;{ }^{1} \mathrm{H} \mathrm{NMR}$ $\left(500 \mathrm{MHz}, \mathrm{CDCl}_{3}\right) \delta$ 7.69-7.61 (m, $\left.4 \mathrm{H}\right), 7.45-7.21(\mathrm{~m}, 16 \mathrm{H}), 4.80\left(\mathrm{~d}, \mathrm{~J}_{\mathrm{AB}}=11.9 \mathrm{~Hz}, 1 \mathrm{H}\right), 4.75\left(\mathrm{~d}, \mathrm{~J}_{\mathrm{AB}}=\right.$ $11.5 \mathrm{~Hz}, 1 \mathrm{H}), 4.71\left(\mathrm{~d}, J_{A B}=11.5 \mathrm{~Hz}, 1 \mathrm{H}\right), 4.45\left(\mathrm{~d}, J_{\mathrm{AB}}=11.9 \mathrm{~Hz}, 1 \mathrm{H}\right), 4.54-4.45(\mathrm{~m}, 1 \mathrm{H})$, 4.30-4.24 (m, $1 \mathrm{H}), 4.10(\mathrm{~d}, J=4.4 \mathrm{~Hz}, 1 \mathrm{H}), 4.10-4.04(\mathrm{~m}, 1 \mathrm{H}), 3.67-3.56(\mathrm{~m}, 1 \mathrm{H}), 3.08(\mathrm{br} \mathrm{s}, 1 \mathrm{H}), 2.87(\mathrm{dd}, J=17.8$, 8.5 Hz, $1 \mathrm{H}), 2.67(\mathrm{dd}, J=18.2,3.7 \mathrm{~Hz}, 1 \mathrm{H}), 2.51(\mathrm{~d}, J=2.2 \mathrm{~Hz}, 1 \mathrm{H}), 1.80-1.65(\mathrm{~m}, 2 \mathrm{H}), 1.58-1.45(\mathrm{~m}, 2$ H), $1.04(\mathrm{~s}, 9 \mathrm{H}), 0.92(\mathrm{t}, J=7.8 \mathrm{~Hz}, 9 \mathrm{H}), 0.82(\mathrm{~d}, J=7.0 \mathrm{~Hz}, 3 \mathrm{H}), 0.57(\mathrm{q}, J=7.8 \mathrm{~Hz}, 6 \mathrm{H}) ;{ }^{13} \mathrm{C} \mathrm{NMR}$ $\left(125 \mathrm{MHz}, \mathrm{CDCl}_{3}\right) \delta 209.1,137.2,137.0,135.5,133.8,133.7,129.6,128.3,128.1,128.0,127.9,127.8$ 127.6, 85.2, 79.0, 76.2, 73.7, 73.4, 70.9, 70.0, 69.4, 60.7, 45.2, 41.0, 36.9, 26.8, 19.1, 7.5, 6.8, 5.2; high resolution mass spectrum (ES+) $\mathrm{m} / \mathrm{z} 815.4159\left[(\mathrm{M}+\mathrm{Na})^{+}\right.$; calcd for $\mathrm{C}_{48} \mathrm{H}_{64} \mathrm{O}_{6} \mathrm{Si}_{2} \mathrm{Na}:$ : 815.4241]. For 17b: IR (neat) $3446,3287,3096,2955,2858,1724,1455,1427,1389,1239,1111 \mathrm{~cm}^{-1} ;[\alpha]_{D}^{20}+45.6(c 0.68$, $\left.\mathrm{CHCl}_{3}\right) ;{ }^{1} \mathrm{H}$ NMR $\left(500 \mathrm{MHz}, \mathrm{CDCl}_{3}\right) \delta$ 7.68-7.62 (m, $\left.4 \mathrm{H}\right), 7.45-7.25(\mathrm{~m}, 16 \mathrm{H}), 4.83\left(\mathrm{~d}, \mathrm{~J}_{\mathrm{AB}}=11.5 \mathrm{~Hz}, 1 \mathrm{H}\right)$, $4.74\left(\mathrm{~d}, J_{\mathrm{AB}}=11.9 \mathrm{~Hz}, 1 \mathrm{H}\right), 4.70\left(\mathrm{~d}, J_{\mathrm{AB}}=11.5 \mathrm{~Hz}, 1 \mathrm{H}\right), 4.51\left(\mathrm{~d}, J_{\mathrm{AB}}=11.9 \mathrm{~Hz}, 1 \mathrm{H}\right), 4.50(\mathrm{dd}, J=5.2$, 2.6 Hz, $1 \mathrm{H}), 4.21(\mathrm{~d}, J=4.8 \mathrm{~Hz}, 1 \mathrm{H})$, 4.15-4.09 (m, $1 \mathrm{H})$, 4.09-3.94 (m, $2 \mathrm{H}), 3.72-3.64(\mathrm{~m}, 2 \mathrm{H}), 2.75$ (dd, $J=16.3,2.9 \mathrm{~Hz}, 1 \mathrm{H}), 2.60(\mathrm{dd}, J=16.3,8.9 \mathrm{~Hz}, 1 \mathrm{H}), 2.56(\mathrm{~d}, J=2.2 \mathrm{~Hz}, 1 \mathrm{H}), 1.75-1.60(\mathrm{~m}, 3 \mathrm{H})$, $1.05(\mathrm{~s}, 9 \mathrm{H}), 0.91(\mathrm{t}, J=7.8 \mathrm{~Hz}, 9 \mathrm{H}), 0.67(\mathrm{~d}, J=7.0 \mathrm{~Hz}, 3 \mathrm{H}), 0.62-0.53(\mathrm{~m}, 6 \mathrm{H}) ;{ }^{13} \mathrm{C} \mathrm{NMR}(125 \mathrm{MHz}$, $\left.\mathrm{CDCl}_{3}\right) \delta 208.9,137.3,137.0,135.5,133.8,133.7,129.6,129.5,128.3,128.2,128.0,127.8,127.7,127.6$ 85.2, 79.3, 76.1, 73.3, 72.1, 71.0, 70.1, 69.9, 60.7, 46.2, 42.8, 35.4, 26.8, 19.1, 12.1, 6.8, 4.9; high resolution mass spectrum (ES+) $m / z 815.4135\left[(\mathrm{M}+\mathrm{Na})^{+}\right.$; calcd for $\mathrm{C}_{48} \mathrm{H}_{64} \mathrm{O}_{6} \mathrm{Si}_{2} \mathrm{Na}:$ : 815.4241]. 


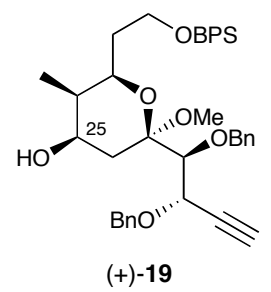

Alcohol (+)-19. To a flask containing (+)-17b $(2.59 \mathrm{~g}, 3.27 \mathrm{mmol}, 1.0$ equiv.) was added dry $\mathrm{MeOH}(33 \mathrm{~mL})$ and $\mathrm{THF}(1.5 \mathrm{~mL})$ and the solution cooled to $0{ }^{\circ} \mathrm{C}$. A pre-made $0.01 \mathrm{M}$ solution of $\mathrm{PPh}_{3} \cdot \mathrm{HBr}$ complex in dry $\mathrm{MeOH}(8.2 \mathrm{~mL}, 0.025$ equiv.) was then added and the resulting mixture stirred at $0{ }^{\circ} \mathrm{C}$ for $7 \mathrm{~h}$ at which time the solution was poured into a flask containing $150 \mathrm{~mL}$ of ether and the resulting mixture extracted with $100 \mathrm{~mL} \mathrm{NaHCO}$, $100 \mathrm{~mL}$ water, and $100 \mathrm{~mL}$ brine. The organic layer was then dried $\left(\mathrm{MgSO}_{4}\right)$ and concentrated to yield a crude oil that was purified by column chromatography. Concentration of the product containing fractions then provided $(+)-19(2.1 \mathrm{~g}, 92 \%$ yield) as a clear, colorless oil: IR (neat) 3447, 3284, 3068, 2930, 2857, 1782, 1471, 1427, 1360, 1209, $1111 \mathrm{~cm}^{-1} ;[\alpha]_{\mathrm{D}}{ }^{20}+55.6\left(c 0.04, \mathrm{CHCl}_{3}\right) ;{ }^{1} \mathrm{H}$ NMR $\left(500 \mathrm{MHz}, \mathrm{CDCl}_{3}\right) \delta$ 7.70-7.64 (m, $\left.4 \mathrm{H}\right), 7.46-7.21(\mathrm{~m}, 16$ $\mathrm{H}), 4.87\left(\mathrm{~d}, J_{\mathrm{AB}}=11.9 \mathrm{~Hz}, 1 \mathrm{H}\right), 4.87\left(\mathrm{~d}, J_{\mathrm{AB}}=11.9 \mathrm{~Hz}, 1 \mathrm{H}\right), 4.78\left(\mathrm{~d}, J_{\mathrm{AB}}=11.9 \mathrm{~Hz}, 1 \mathrm{H}\right), 4.52\left(\mathrm{~d}, J_{\mathrm{AB}}=\right.$ $11.9 \mathrm{~Hz}, 1 \mathrm{H}$ ), 4.39 (dd, $J=2.2,2.2 \mathrm{~Hz}, 1 \mathrm{H}), 4.12-4.07(\mathrm{~m}, 1 \mathrm{H}), 3.89$ (ddd, $J=8.9,4.4,2.2 \mathrm{~Hz}, 1 \mathrm{H}$ ), 3.85-3.73 (m, $3 \mathrm{H}), 3.01(\mathrm{~s}, 3 \mathrm{H}), 2.57(\mathrm{~d}, J=2.2 \mathrm{~Hz}, 1 \mathrm{H}), 2.07-2.01(\mathrm{~m}, 2 \mathrm{H}), 1.87-1.73(\mathrm{~m}, 3 \mathrm{H}), 1.67-$ $1.60(\mathrm{~m}, 1 \mathrm{H}), 1.06(\mathrm{~s}, 9 \mathrm{H}), 0.83(\mathrm{~d}, J=7.0 \mathrm{~Hz}, 3 \mathrm{H}) ;{ }^{13} \mathrm{C} \operatorname{NMR}\left(125 \mathrm{MHz}, \mathrm{CDCl}_{3}\right) \delta$ 138.8, 137.7, 135.5, 133.9, 133.8, 129.6, 128.3, 128.2, 128.0, 127.9, 127.8, 127.7, 127.6, 127.1, 101.2, 81.8, 79.8, 74.4, 70.4, $69.4,68.4,67.8,60.6,47.8,37.7,35.7,31.7,26.8,19.2,4.0$; high resolution mass spectrum (ES+) $\mathrm{m} / \mathrm{z}$ $715.3533\left[(\mathrm{M}+\mathrm{Na})^{+}\right.$; calcd for $\left.\mathrm{C}_{43} \mathrm{H}_{52} \mathrm{O}_{6} \mathrm{SiNa}: 715.3444\right]$.

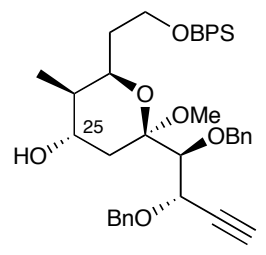

$(+)-18$

Alcohol (+)-18: The same procedure as used for alcohol (+)-19 was performed using the following material: (+)-17a $\left(5.18 \mathrm{~g}, 6.53 \mathrm{mmol}, 1.0\right.$ equiv.) in $\mathrm{MeOH}(65 \mathrm{~mL})$ and $\mathrm{THF}(3 \mathrm{~mL})$ at $0{ }^{\circ} \mathrm{C}$. Added $\mathrm{PPh}_{3} \cdot \mathrm{HBr}(16 \mathrm{~mL}, 0.025$ equiv., $0.01 \mathrm{M}$ solution in $\mathrm{MeOH})$ and mixed at $0{ }^{\circ} \mathrm{C}$ for $7 \mathrm{~h}$. Work-up as 
described above provided (+)-18 (3.7 g, 82\% yield) as a clear, colorless oil: IR (neat) 3529, 3287, 3030, $2930,2857,1496,1454,1427,1359,1217,1111,1064,1028 \mathrm{~cm}^{-1} ;[\alpha]_{\mathrm{D}}{ }^{20}+66.1\left(c 0.05, \mathrm{CHCl}_{3}\right) ;{ }^{1} \mathrm{H} \mathrm{NMR}$ $\left(500 \mathrm{MHz}, \mathrm{CDCl}_{3}\right) \delta$ 7.70-7.64 (m, $\left.4 \mathrm{H}\right), 7.46-7.21(\mathrm{~m}, 16 \mathrm{H}), 4.86\left(\mathrm{~d}, \mathrm{~J}_{\mathrm{AB}}=12.2 \mathrm{~Hz}, 1 \mathrm{H}\right), 4.84\left(\mathrm{~d}, \mathrm{~J}_{\mathrm{AB}}=\right.$ $11.9 \mathrm{~Hz}, 1 \mathrm{H}), 4.75\left(\mathrm{~d}, J_{\mathrm{AB}}=11.9 \mathrm{~Hz}, 1 \mathrm{H}\right), 4.50\left(\mathrm{~d}, J_{\mathrm{AB}}=12.9 \mathrm{~Hz}, 1 \mathrm{H}\right), 4.33(\mathrm{dd}, J=2.2,2.2 \mathrm{~Hz}, 1 \mathrm{H})$, 4.28-4.23 (m, $1 \mathrm{H}), 3.84-3.74(\mathrm{~m}, 4 \mathrm{H}), 3.71(\mathrm{~d}, J=2.2 \mathrm{~Hz}, 1 \mathrm{H}), 3.02(\mathrm{~s}, 3 \mathrm{H}), 2.57(\mathrm{~d}, J=2.2 \mathrm{~Hz}, 1 \mathrm{H})$, 2.31-2.26 (m, $1 \mathrm{H}), 1.84-1.58(\mathrm{~m}, 4 \mathrm{H}), 1.05(\mathrm{~s}, 9 \mathrm{H}), 0.83(\mathrm{~d}, \mathrm{~J}=7.0 \mathrm{~Hz}, 3 \mathrm{H}) ;{ }^{13} \mathrm{C} \mathrm{NMR}\left(125 \mathrm{MHz}, \mathrm{CDCl}_{3}\right)$ $\delta 138.6,137.5,135.5,133.9,133.8,129.6,128.3,128.1,128.0,127.9,127.8,127.6,127.2,101.7,81.5$ $79.7,74.5,70.4,69.2,63.4,60.6,47.7,37.4,35.7,28.7,26.8,19.1,10.6$; high resolution mass spectrum (ES+) $m / z 715.3449\left[(\mathrm{M}+\mathrm{Na})^{+} ;\right.$calcd for $\left.\mathrm{C}_{43} \mathrm{H}_{52} \mathrm{O}_{6} \mathrm{SiNa}: 715.3533\right]$

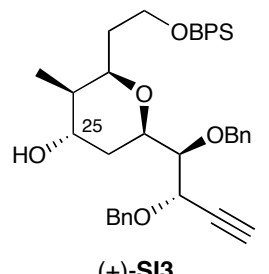

Alcohol (+)-SI3 : To a solution of (+)-18 (0.312 g, $0.450 \mathrm{mmol}, 1.0$ equiv.) in dichloromethane (9 $\mathrm{mL}$ ) cooled to $-78{ }^{\circ} \mathrm{C}$ was added triethylsilane (1.5 mL, 20 equiv.). At this time TMSOTf (1.4 mL, 3.0 equiv., 1.0 M solution in dichloromethane) was added dropwise and the reaction was immediately quenched by rapid injection of $5 \mathrm{~mL}$ of sat. $\mathrm{NH}_{4} \mathrm{Cl}$. The resulting mixture was allowed to warm to rt and an additional $5 \mathrm{~mL}$ of water was then added. Separation of the organic layer was followed by washing of the aqueous phase with $3 \times 50 \mathrm{~mL}$ of dichloromethane. The combined organic layers were then dried $\left(\mathrm{MgSO}_{4}\right)$ and concentrated to yield a crude oil. Purification was then accomplished by column chromatography using 10\% EtOAc/Hexanes to $30 \%$ EtOAc/Hexanes to provide (+)-SI3 (0.28 g, 93\% yield) as a clear, colorless oil: IR (neat) 3030, 2956, 2880, 1959, 1496, 1455, 1390, 1350, 1208, 1110, 1070, $1028 \mathrm{~cm}^{-1} ;[\alpha]_{D}{ }^{20}+56.4\left(c 1.25, \mathrm{CHCl}_{3}\right) ;{ }^{1} \mathrm{H}$ NMR $\left(500 \mathrm{MHz}, \mathrm{CDCl}_{3}\right) \delta$ 7.78-7.71 (m, $\left.4 \mathrm{H}\right), 7.50-7.29$ $(\mathrm{m}, 16 \mathrm{H}), 4.92\left(\mathrm{~d}, J_{\mathrm{AB}}=11.5 \mathrm{~Hz}, 1 \mathrm{H}\right), 4.89\left(\mathrm{~d}, J_{\mathrm{AB}}=11.9 \mathrm{~Hz}, 1 \mathrm{H}\right), 4.73\left(\mathrm{~d}, J_{\mathrm{AB}}=11.5 \mathrm{~Hz}, 1 \mathrm{H}\right), 4.46(\mathrm{~d}$, $\left.J_{\mathrm{AB}}=11.5 \mathrm{~Hz}, 1 \mathrm{H}\right), 4.41(\mathrm{dd}, J=4.0,2.2 \mathrm{~Hz}, 1 \mathrm{H}), 4.10-4.06(\mathrm{~m}, 1 \mathrm{H}), 4.00-3.91(\mathrm{~m}, 2 \mathrm{H}), 3.81-3.75(\mathrm{~m}, 2$ H), $3.70(\mathrm{dd}, J=6.7,4.4 \mathrm{~Hz}, 1 \mathrm{H}), 2.49(\mathrm{~d}, J=2.2 \mathrm{~Hz}, 1 \mathrm{H}), 1.82-1.55(\mathrm{~m}, 5 \mathrm{H}), 1.11(\mathrm{~s}, 9 \mathrm{H}), 0.89(\mathrm{~d}, J=$ $7.0 \mathrm{~Hz}, 3 \mathrm{H}) ;{ }^{13} \mathrm{C}$ NMR $\left(125 \mathrm{MHz}, \mathrm{CDCl}_{3}\right) \delta 138.6,137.8,135.5,134.0,129.5,128.4,128.3,128.2,128.1$, 
$127.9,127.7,127.6,127.4,82.3,80.1,75.2,74.3,71.9,71.0,70.8,70.4,61.1,38.3,35.8,29.7,26.9$, 19.2, 11.0; high resolution mass spectrum (ES+) $\mathrm{m} / \mathrm{z} 685.3339\left[(\mathrm{M}+\mathrm{Na})^{+}\right.$; calcd for $\mathrm{C}_{42} \mathrm{H}_{50} \mathrm{O}_{5} \mathrm{SiNa}$ : 685.3428].

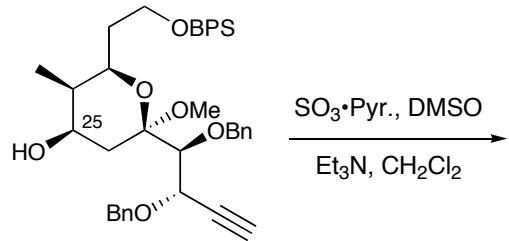

$(+)-19$

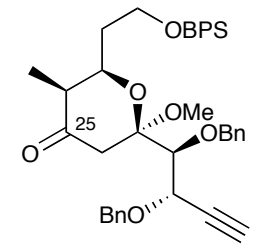

$(+)$-SI4

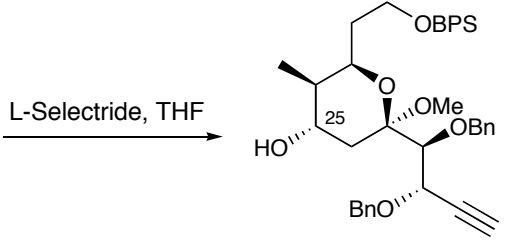

$(+)-18$

Ketone (+)-SI4. Alcohol (+)-19 (0.42 g, 0.60 mmol, 1.0 equiv.) was dissolved in dichloromethane $(60 \mathrm{~mL})$ and to this solution was sequentially added dimethylsulfoxide $(5 \mathrm{~mL})$, triethylamine $(0.42 \mathrm{~mL}, 5.0$ equiv.), and $\mathrm{SO}_{3} \cdot \operatorname{Pyr}(0.21 \mathrm{~g}, 2.2$ equiv.). The resulting solution was mixed at $\mathrm{rt}$ for several hours until starting material was consumed as judged by TLC. The usual work-up then provided a crude oil that was purified by column chromatography (10\% EtOAc/Hexanes) to provide (+)-S14 $(0.40 \mathrm{~g}, 95 \%$ yield $)$ as a clear, colorless oil: IR (neat) 3281, 2929, 2857, 1779, 1719, 1458, 1389, 1359, 1215, $1111 \mathrm{~cm}^{-1} ;[\alpha]_{\mathrm{D}}^{20}$ $+54.2\left(c 0.32, \mathrm{CHCl}_{3}\right) ;{ }^{1} \mathrm{H}$ NMR $\left(500 \mathrm{MHz}, \mathrm{CDCl}_{3}\right) \delta$ 7.68-7.64 (m, $\left.4 \mathrm{H}\right), 7.46-7.24(\mathrm{~m}, 16 \mathrm{H}), 4.96\left(\mathrm{~d}, \mathrm{~J}_{\mathrm{AB}}=\right.$ $11.9 \mathrm{~Hz}, 1 \mathrm{H}), 4.88\left(\mathrm{~d}, J_{A B}=11.9 \mathrm{~Hz}, 1 \mathrm{H}\right), 4.82\left(\mathrm{~d}, J_{\mathrm{AB}}=11.9 \mathrm{~Hz}, 1 \mathrm{H}\right), 4.51\left(\mathrm{~d}, J_{A B}=11.9 \mathrm{~Hz}, 1 \mathrm{H}\right), 4.35$ (dd, $J=2.2,2.2 \mathrm{~Hz}, 1 \mathrm{H}), 4.25-4.20(\mathrm{~m}, 1 \mathrm{H}), 3.94(\mathrm{~d}, J=2.2 \mathrm{~Hz}, 1 \mathrm{H}), 3.85-3.74(\mathrm{~m}, 2 \mathrm{H}), 3.20(\mathrm{~d}, J=$ $15.2 \mathrm{~Hz}, 1 \mathrm{H}), 3.02(\mathrm{~s}, 3 \mathrm{H}), 2.59(\mathrm{~d}, J=1.8 \mathrm{~Hz}, 1 \mathrm{H}), 2.40$ (d, J=15.2 Hz, $1 \mathrm{H}), 2.31-2.25(\mathrm{~m}, 1 \mathrm{H}), 1.86$ (dddd, $J=13.7,9.3,4.8,4.8 \mathrm{~Hz}, 1 \mathrm{H}), 1.68-1.58(\mathrm{~m}, 1 \mathrm{H}), 1.06(\mathrm{~d}, J=6.3 \mathrm{~Hz}, 3 \mathrm{H}), 1.06(\mathrm{~s}, 9 \mathrm{H}) ;{ }^{13} \mathrm{C}$ NMR $\left(125 \mathrm{MHz}, \mathrm{CDCl}_{3}\right) \delta 210.2,138.5,137.4,135.5,133.6,129.7,128.4,128.3,128.2,128.1,128.0$, $127.8,127.7,127.4,102.8,81.4,79.3,76.7,74.5,70.5,69.1,68.0,60.2,48.2,47.9,42.0,34.7,26.8$, 19.1, 10.3; high resolution mass spectrum (ES+) $\mathrm{m} / \mathrm{z} 713.3296\left[(\mathrm{M}+\mathrm{Na})^{+}\right.$; calcd for $\mathrm{C}_{43} \mathrm{H}_{50} \mathrm{O}_{6} \mathrm{SiNa}$ : 713.3377].

Alcohol (+)-18: To a flask containing ketone (+)-SI4 (0.30 g, $0.43 \mathrm{mmol}, 1.0$ equiv.) was added THF $(4.3 \mathrm{~mL})$ and the resulting solution cooled to $-78{ }^{\circ} \mathrm{C}$. A $1.0 \mathrm{M}$ solution of L-selectride in THF $(0.60$ $\mathrm{mL}, 1.3$ equiv.) was then added down the side of the flask and the reaction was allowed to stir overnight at $-78{ }^{\circ} \mathrm{C}$. The next morning the reaction was quenched by addition of $2 \mathrm{~mL}$ of saturated $\mathrm{NH}_{4} \mathrm{Cl}$ and allowed to warm to rt. The layers were separated and the aqueous was washed with $2 \times 10 \mathrm{~mL}$ of EtOAc. 
The combined organics were then dried $\left(\mathrm{MgSO}_{4}\right)$ and concentrated to yield a crude oil that was purified by column chromatography ( $20 \%$ EtOAc/Hexanes). The product containing fractions were collected and concentrated to provide alcohol (+)-18 (0.28 g, 94\% yield) as a clear, colorless oil.

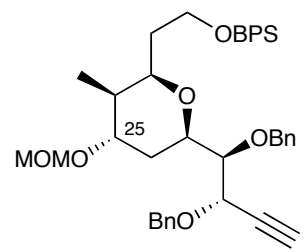

$(+)-20$

Alkyne (+)-20: To a solution of alcohol (+)-SI3 (0.312 g, $0.471 \mathrm{mmol}, 1.0$ equiv.) in acetonitrile (4.0 mL) was added 2,6-lutidine (0.22 mL, 4.0 equiv.) followed by $\mathrm{MOMCl}(0.11 \mathrm{~mL}, 3.0$ equiv.). The resulting reaction mixture was then allowed to mix overnight at $\mathrm{rt}$ at which point it was quenched by the addition of $5 \mathrm{~mL}$ of sat. $\mathrm{NaHCO}_{3}$ and $50 \mathrm{~mL}$ of EtOAc. The organic phase was then separated and the aqueous phase was washed by $2 \times 50 \mathrm{~mL}$ of EtOAc. The combined organics were dried $\left(\mathrm{MgSO}_{4}\right)$ and concentrated to yield a crude oil. Purification was accomplished by column chromatography (10\% EtOAc/Hexanes) to provide (+)-20 (0.33 g, 100\% yield) as a clear, colorless oil: IR (neat) 3288, 3030, $2929,2884,2113,1958,1588,1496,1469,1428,1349,1308,1209,1109,1039 \mathrm{~cm}^{-1} ;[\alpha]_{D}^{20}+42.4(c$ 4.80, $\left.\mathrm{CHCl}_{3}\right) ;{ }^{1} \mathrm{H}$ NMR $\left(500 \mathrm{MHz}, \mathrm{CDCl}_{3}\right) \delta$ 7.81-7.74 (m, $\left.4 \mathrm{H}\right), 7.52-7.28(\mathrm{~m}, 16 \mathrm{H}), 4.96\left(\mathrm{~d}, \mathrm{~J}_{\mathrm{AB}}=11.5 \mathrm{~Hz}\right.$, $1 \mathrm{H}), 4.93\left(\mathrm{~d}, J_{\mathrm{AB}}=11.5 \mathrm{~Hz}, 1 \mathrm{H}\right), 4.79\left(\mathrm{~d}, J_{\mathrm{AB}}=11.5 \mathrm{~Hz}, 1 \mathrm{H}\right), 4.73(\mathrm{br} \mathrm{s}, 2 \mathrm{H}), 4.51\left(\mathrm{~d}, J_{\mathrm{AB}}=11.5 \mathrm{~Hz}, 1\right.$ H), $4.47(\mathrm{dd}, J=4.0,2.2 \mathrm{~Hz}, 1 \mathrm{H}), 4.15-4.12(\mathrm{~m}, 2 \mathrm{H}), 4.05-3.99(\mathrm{~m}, 1 \mathrm{H}), 3.88-3.79(\mathrm{~m}, 3 \mathrm{H}), 3.75-3.73$ (m, $1 \mathrm{H}), 3.41(\mathrm{~s}, 3 \mathrm{H}), 2.53(\mathrm{~d}, J=2.2 \mathrm{~Hz}, 1 \mathrm{H}), 1.86-1.74(\mathrm{~m}, 2 \mathrm{H}), 1.70-1.61(\mathrm{~m}, 2 \mathrm{H}), 1.15(\mathrm{~s}, 9 \mathrm{H}), 0.96$ (d, $J=7.4 \mathrm{~Hz}, 3 \mathrm{H}) ;{ }^{13} \mathrm{C}$ NMR $\left(125 \mathrm{MHz}, \mathrm{CDCl}_{3}\right) \delta 138.7,137.9,135.6,134.2,134.1,129.5,128.3,128.2$, $128.1,127.9,127.6,127.5,94.8,82.5,80.3,75.5,75.3,74.4,72.6,71.4,71.1,71.0,61.2,55.3,36.4$, 36.0, 27.2, 26.9, 19.3, 11.1; high resolution mass spectrum (ES+) $\mathrm{m} / \mathbf{z} 729.3573\left[(\mathrm{M}+\mathrm{Na})^{+}\right.$; calcd for $\left.\mathrm{C}_{44} \mathrm{H}_{54} \mathrm{O}_{6} \mathrm{SiNa}: 729.2690\right]$. 


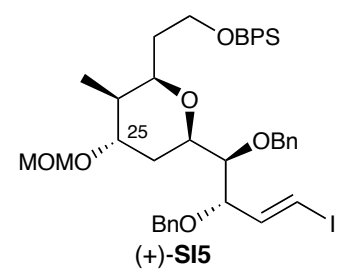

Vinyl lodide (+)-SI5. To a solution of alkyne (+)-20 (0.32 g, $0.45 \mathrm{mmol}, 1.0$ equiv.) in dichloromethane $(4.5 \mathrm{~mL})$ was added Schwartz's reagent $(0.58 \mathrm{~g}, 5.0$ equiv.) and the resulting cloudy white solution was allowed to mix at rt until a homogenous yellow solution was obtained (ca. $1 \mathrm{hr}$ ). The resulting yellow solution was allowed to mix for another $30 \mathrm{~min}$ at $\mathrm{rt}$, slowly becoming orange in color, at which point solid $\mathrm{I}_{2}$ (0.15 g, 1.3 equiv.) was added. The resulting solution immediately turned yellow and eventually became deep red/brown in color. After $30 \mathrm{~min}$, the reaction was quenched with $30 \mathrm{~mL}$ of sat. $\mathrm{NaHCO}_{3}$ and the organic phase separated. The aqueous phase was then washed with $3 \times 50 \mathrm{~mL}$ of dichloromethane and the combined organics were dried $\left(\mathrm{MgSO}_{4}\right)$ and concentrated to yield a crude yellow oil. Purification was accomplished by column chromatography (5\% EtOAc/Hexanes to $20 \%$ EtOAc/Hexanes) to provide (+)-SI5 (0.38 g, 100\% yield) as a clear yellow oil: IR (neat) 3067, 3030, 2929, $2883,1603,1454,1427,1358,1108,1071,1039 \mathrm{~cm}^{-1} ;[\alpha]_{D}^{20}+25.4\left(c 3.30, \mathrm{CHCl}_{3}\right) ;{ }^{1} \mathrm{H} \mathrm{NMR}(500 \mathrm{MHz}$, $\left.\mathrm{CDCl}_{3}\right) \delta$ 7.73-7.68 (m, $\left.4 \mathrm{H}\right), 7.46-7.26(\mathrm{~m}, 16 \mathrm{H}), 6.60(\mathrm{dd}, J=14.8,8.1 \mathrm{~Hz}, 1 \mathrm{H}), 6.19(\mathrm{~d}, J=14.8 \mathrm{~Hz}, 1$ $\mathrm{H}), 4.78\left(\mathrm{~d}, J_{\mathrm{AB}}=11.5 \mathrm{~Hz}, 1 \mathrm{H}\right), 4.67\left(\mathrm{~d}, J_{\mathrm{AB}}=11.5 \mathrm{~Hz}, 1 \mathrm{H}\right), 4.67(\mathrm{br} \mathrm{s}, 2 \mathrm{H}), 4.55\left(\mathrm{~d}, J_{\mathrm{AB}}=11.9 \mathrm{~Hz}, 1 \mathrm{H}\right)$, $4.29\left(\mathrm{~d}, J_{A B}=11.9 \mathrm{~Hz}, 1 \mathrm{H}\right), 3.99-3.94(\mathrm{~m}, 1 \mathrm{H}), 3.93(\mathrm{dd}, J=8.1,4.0 \mathrm{~Hz}, 1 \mathrm{H}), 3.81-3.70(\mathrm{~m}, 4 \mathrm{H}), 3.58$ (dd, $J=6.3,4.4 \mathrm{~Hz}, 1 \mathrm{H}), 3.35(\mathrm{~s}, 3 \mathrm{H}), 1.85-1.55(\mathrm{~m}, 5 \mathrm{H}), 1.09(\mathrm{~s}, 9 \mathrm{H}), 0.91(\mathrm{~d}, J=7.4 \mathrm{~Hz}, 3 \mathrm{H}) ;{ }^{13} \mathrm{C}$ NMR $\left(125 \mathrm{MHz}, \mathrm{CDCl}_{3}\right) \delta 143.5,138.6,138.1,135.5,135.4,134.0,133.9,133.8,129.6,128.3,128.2$ $128.1,127.7,127.6,127.5,94.8,82.5,80.0,75.5,74.4,72.5,71.5,70.8,61.5,55.3,36.2,36.0,27.2$, 26.9, 19.2, 11.1; high resolution mass spectrum (ES+) $\mathrm{m} / 2$ 857.2717 $\left[(\mathrm{M}+\mathrm{Na})^{+}\right.$; calcd for $\mathrm{C}_{44} \mathrm{H}_{55} \mathrm{IO}_{6} \mathrm{SiNa}$ : 857.2813].

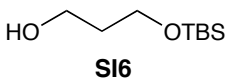

Alcohol SI6. To a $0{ }^{\circ} \mathrm{C}$ solution of distilled 1,3-propanediol (11.4 g, 1.1 equiv.) in THF (335 mL) was added $n$-BuLi (59.9 mL, $2.5 \mathrm{M}$ in hexanes, 1.1 equiv.) dropwise via addition funnel. After $1 \mathrm{~h}, \mathrm{a}$ solution of tert-butyldimethylsilyl chloride $(20.9 \mathrm{~g}, 136 \mathrm{mmole})$ in THF $(40 \mathrm{~mL})$ was added via cannula and 
the reaction was allowed to warm to ambient temperature. After $15 \mathrm{~h}$, the reaction was quenched with water $(50 \mathrm{~mL})$ and concentrated in vacuo. The residue was then extracted with $\mathrm{CH}_{2} \mathrm{Cl}_{2}(250 \mathrm{~mL})$, and the aqueous phase was washed with $\mathrm{CH}_{2} \mathrm{Cl}_{2}(3 \times 50 \mathrm{~mL})$. The combined organic layers were then washed with brine $(50 \mathrm{~mL})$, dried over $\mathrm{MgSO}_{4}$ and concentrated in vacuo to afford a yellow oil. Purification by flash chromatography (4/1 hexanes/EtOAc) afforded $25.0 \mathrm{~g}$ (97\% yield) of SI6 as a pale yellow oil: $\mathrm{R}_{\mathrm{f}} 0.26$ (4/1 hexanes/EtOAc); IR (thin film, $\mathrm{CDCl}_{3}$ ) 3355 (br, m), 2955 (s), 2928 (s), 2857 (s), 1472 (m), 1256 (s), 1097 (s) $\mathrm{cm}^{-1} ;{ }^{1} \mathrm{H}$ NMR $\left(500 \mathrm{MHz}, \mathrm{CDCl}_{3}\right) \delta 3.82(\mathrm{t}, J=5.6 \mathrm{~Hz}, 2 \mathrm{H}), 3.78(\mathrm{q}, J=5.4 \mathrm{~Hz}, 2 \mathrm{H}), 2.59(\mathrm{t}, J=$ $5.3 \mathrm{~Hz}, 1 \mathrm{H}$ ), 1.76 (quint, J= 5.6 Hz, $2 \mathrm{H}$ ), $0.89(\mathrm{~s}, 9 \mathrm{H}), 0.06(\mathrm{~s}, 6 \mathrm{H}) ;{ }^{13} \mathrm{C} \mathrm{NMR}\left(125 \mathrm{MHz}, \mathrm{CDCl}_{3}\right) \delta 62.7$, 62.2, 34.2, 25.8, 18.1, -5.5; high resolution mass spectrum (ES+) m/z $213.1280\left[(\mathrm{M}+\mathrm{Na})^{+}\right.$; calcd for $\mathrm{C}_{9} \mathrm{H}_{22}$ $\left.\mathrm{O}_{2} \mathrm{SiNa}^{+}: 213.1388\right]$.

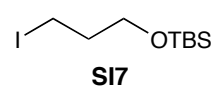

lodide SI7. To a flask containing alcohol SI6 (1.7 g, 1.2 equiv.) was added dichloromethane (73 $\mathrm{mL}$ ). The solution was cooled to $0{ }^{\circ} \mathrm{C}$ and triphenylphosphine $(1.9 \mathrm{~g}, 7.3 \mathrm{mmol}, 1.0$ equiv.) followed by imidazole ( $0.75 \mathrm{~g}, 1.5$ equiv.) were added. lodine ( $1.9 \mathrm{~g}, 1.05$ equiv.) was then added and the ice bath removed. The reaction was allowed to mix for $2 \mathrm{~h}$ at which time a $1: 1$ solution of $10 \% \mathrm{Na}_{2} \mathrm{SO}_{3}$ : saturated $\mathrm{NaHCO}_{3}(50 \mathrm{~mL})$ was added. The organic layer was separated and the aqueous phase washed with $2 \mathrm{x}$ $25 \mathrm{~mL}$ of dichloromethane. The combined organics were dried $\left(\mathrm{MgSO}_{4}\right)$ and concentrated to yield a crude oil which was purified by column chromatography (5\% EtOAc/Hexanes) to provide SI7 as a clear, colorless oil. All spectra data matched that in the literature. ${ }^{3}$

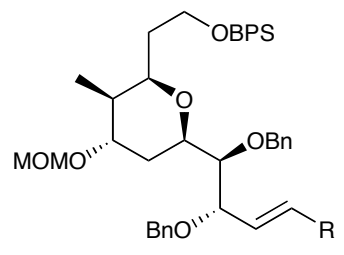

$(+)-22, \mathrm{R}=\left(\mathrm{CH}_{2}\right)_{3} \mathrm{OTBS}$

Suzuki-Miyaura coupling [(+)-22]. To a flask containing SI7 (0.40 g, 3.0 equiv.) was added dry ether $(4.9 \mathrm{~mL})$ and the reaction flask was cooled to $-78{ }^{\circ} \mathrm{C}$. To this solution was added $t$-BuLi $(1.8 \mathrm{~mL}$, 6.0 equiv., $1.5 \mathrm{M}$ in pentane) and the resulting anion was stirred for $10 \mathrm{~min}$. at which point 9-BBN-OMe 
(2.7 mL, 6.0 equiv., $1.0 \mathrm{M}$ solution in hexanes) was added and the reaction was stirred at $-78^{\circ} \mathrm{C}$ for 10 min before being warmed to rt and allowed to stir for $1 \mathrm{~h}$. An aqueous solution of $\mathrm{Cs}_{2} \mathrm{CO}_{3}(0.44 \mathrm{~mL}, 3.0$ equiv., 3.0 $\mathrm{M}$ aq. solution) was then added followed by a solution of (+)-SI5 (0.37 g, $0.44 \mathrm{mmol}, 1.0$ equiv.) in DMF (4.9 mL). The $\mathrm{Pd}(\mathrm{dppf})_{2} \mathrm{Cl}_{2}(0.02 \mathrm{~g}, 0.05$ equiv.) was then added and the resulting mixture was allowed to stir at rt overnight. The reaction was quenched by addition of $10 \mathrm{~mL}$ of sat. $\mathrm{NaHCO}_{3}$ and $20 \mathrm{~mL}$ of $\mathrm{H}_{2} \mathrm{O}$. The organic layer was separated and the aqueous layer washed with $2 \times 25 \mathrm{~mL}$ of ether. The combined organic layers were washed with $50 \mathrm{~mL}$ of $\mathrm{H}_{2} \mathrm{O}$ and $50 \mathrm{~mL}$ of brine then dried $\left(\mathrm{MgSO}_{4}\right)$ and concentrated to yield a crude oil. Purification was accomplished using careful column chromatography (5\% EtOAc/Hexanes) to provide (+)-22 (0.39 g, 100\% yield) as a clear, colorless oil: IR (neat) 3378, 3067, 2927, 2857, 1451, 1411, 1361, 1300, 1253, 1106, $1039 \mathrm{~cm}^{-1} ;[\alpha]_{D}^{20}+12.2\left(c 1.22, \mathrm{CHCl}_{3}\right) ;{ }^{1} \mathrm{H}$ NMR $(500$ $\left.\mathrm{MHz}, \mathrm{CDCl}_{3}\right) \delta$ 7.71-7.66 (m, $\left.4 \mathrm{H}\right), 7.43-7.22(\mathrm{~m}, 16 \mathrm{H}), 5.56(\mathrm{ddd}, J=15.2,6.3,6.3 \mathrm{~Hz}, 1 \mathrm{H}), 5.49(\mathrm{dd}, J$ = 15.2, 8.1 Hz, $1 \mathrm{H}), 4.78\left(\mathrm{~d}, \mathrm{~J}_{\mathrm{AB}}=11.5 \mathrm{~Hz}, 1 \mathrm{H}\right), 4.69\left(\mathrm{~d}, \mathrm{~J}_{\mathrm{AB}}=11.5 \mathrm{~Hz}, 1 \mathrm{H}\right), 4.66\left(\mathrm{~d}, \mathrm{~J}_{\mathrm{AB}}=7.0 \mathrm{~Hz}, 1 \mathrm{H}\right)$, $4.64\left(\mathrm{~d}, J_{\mathrm{AB}}=7.0 \mathrm{~Hz}, 1 \mathrm{H}\right), 4.55\left(\mathrm{~d}, J_{\mathrm{AB}}=11.9 \mathrm{~Hz}, 1 \mathrm{H}\right), 4.26\left(\mathrm{~d}, J_{\mathrm{AB}}=11.9 \mathrm{~Hz}, 1 \mathrm{H}\right), 3.96-3.91(\mathrm{~m}, 1 \mathrm{H})$, $3.88(\mathrm{dd}, J=8.1,4.4 \mathrm{~Hz}, 1 \mathrm{H}), 3.84-3.71(\mathrm{~m}, 3 \mathrm{H}), 3.63-3.57(\mathrm{~m}, 2 \mathrm{H}), 3.33(\mathrm{~s}, 3 \mathrm{H}), 2.10$ (ddd, $J=6.7$, 6.7, $6.7 \mathrm{~Hz}, 2 \mathrm{H}), 1.85-1.53(\mathrm{~m}, 9 \mathrm{H}), 1.07(\mathrm{~s}, 9 \mathrm{H}), 0.93(\mathrm{~s}, 9 \mathrm{H}), 0.92(\mathrm{~d}, J=5.9 \mathrm{~Hz}, 3 \mathrm{H}), 0.06(\mathrm{~s}, 6 \mathrm{H})$; ${ }^{13} \mathrm{C}$ NMR $\left(125 \mathrm{MHz}, \mathrm{CDCl}_{3}\right) \delta 142.8,142.7,139.3,139.0,137.5,132.6,131.3,131.2,131.1,130.6,130.4$, $130.3,130.2,130.1,95.8,83.8,81.4,76.7,75.5,74.2,72.6,71.1,69.6,61.8,60.8,54.0,34.0,33.7,29.8$ 26.0, 23.9, 23.0, 15.8, 14.9, 7.2, -10.1; high resolution mass spectrum (ES+) $m / z$ 903,5050 [(M+Na) calcd for $\left.\mathrm{C}_{53} \mathrm{H}_{76} \mathrm{O}_{7} \mathrm{Si}_{2} \mathrm{Na}: 903.5130\right]$.

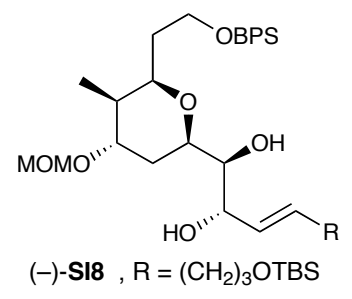

Diol (-)-S18. To a solution of (+)-22 (0.032 g, 0.036 mmol, 1.0 equiv.) was added THF $(0.7 \mathrm{~mL})$ and the resulting solution was cooled to $-78{ }^{\circ} \mathrm{C}$. A pre-made $1.0 \mathrm{M}$ solution of LiDBB in THF was then added dropwise until a deep blue color persisted at which time the reaction was allowed to warm to -40 ${ }^{\circ} \mathrm{C}$. The reaction became red in color and more of the $1.0 \mathrm{M}$ solution of LiDBB was added once again 
until a deep blue color persisted for $30 \mathrm{~min}$. The reaction was then cooled back to $-78{ }^{\circ} \mathrm{C}$ and quenched by the addition of $5 \mathrm{~mL}$ of sat. $\mathrm{NH}_{4} \mathrm{Cl}$ and allowed to warm to rt. Purification was accomplished by column chromatography (20\% EtOAc/Hexanes to $40 \%$ EtOAc/Hexanes) to provide (-)-SI8 (0.022 g, $88 \%$ yield) as a clear, colorless oil: IR (neat) $3456,2928,2856,1471,1428,1360,1255,1104,1039 \mathrm{~cm}^{-1} ;[\alpha]_{D}^{20}-8.4$ (c 0.35, $\left.\mathrm{CHCl}_{3}\right) ;{ }^{1} \mathrm{H}$ NMR $\left(500 \mathrm{MHz}, \mathrm{CDCl}_{3}\right) \delta$ 7.67-7.62 (m, $\left.4 \mathrm{H}\right)$, 7.42-7.31 (m, $\left.6 \mathrm{H}\right), 5.70$ (ddd, J= 15.7, 6.8, $6.8 \mathrm{~Hz}, 1 \mathrm{H}), 5.50(\mathrm{dd}, J=15.7,7.1 \mathrm{~Hz}, 1 \mathrm{H}), 4.65(\mathrm{br} \mathrm{s}, 2 \mathrm{H}), 4.14-4.02(\mathrm{~m}, 2 \mathrm{H}), 3.77-3.66(\mathrm{~m}, 4 \mathrm{H})$, $3.57(\operatorname{app~t}, J=6.6 \mathrm{~Hz}, 2 \mathrm{H}), 3.42-3.36(\mathrm{~m}, 1 \mathrm{H}), 3.33(\mathrm{~s}, 3 \mathrm{H}), 2.72(\mathrm{~d}, J=2.5 \mathrm{~Hz}, 1 \mathrm{H}), 2.07$ (ddd, $J=$ 6.9, 6.9, $6.9 \mathrm{~Hz}, 2 \mathrm{H}), 1.94(\mathrm{~d}, J=3.6 \mathrm{~Hz}, 1 \mathrm{H}), 1.85-1.80(\mathrm{~m}, 1 \mathrm{H}), 1.76-1.63(\mathrm{~m}, 3 \mathrm{H}), 1.60-1.52(\mathrm{~m}, 3 \mathrm{H})$, $1.03(\mathrm{~s}, 9 \mathrm{H}), 0.87(\mathrm{~s}, 9 \mathrm{H}), 0.86(\mathrm{~d}, J=4.0 \mathrm{~Hz}, 3 \mathrm{H}),-0.05(\mathrm{~s}, 6 \mathrm{H}) ;{ }^{13} \mathrm{C} \mathrm{NMR}\left(125 \mathrm{MHz}, \mathrm{CDCl}_{3}\right) \delta 139.0$, $138.2,138.1,137.3,132.7,131.8,130.6,96.0,75.2,75.0,74.6,71.3,61.6,60.0,54.1,33.7,33.4,29.6$, 25.9, 25.1, 23.9, 22.9, 15.8, 7.0, -10.1; high resolution mass spectrum (ES+) $m / z 723.4086\left[(\mathrm{M}+\mathrm{Na})^{+}\right.$; calcd for $\left.\mathrm{C}_{39} \mathrm{H}_{64} \mathrm{O}_{7} \mathrm{Si}_{2} \mathrm{Na}: 723.4191\right]$.

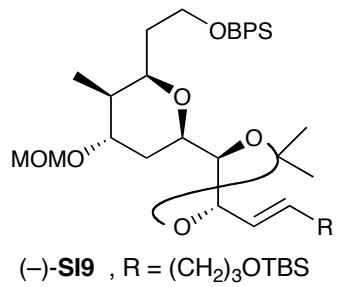

Acetonide (-)-SI9. To a flask containing (-)-SI8 (0.144 g, $0.206 \mathrm{mmol}, 1.0$ equiv.) was added 2,2-dimethoxypropane $(3 \mathrm{~mL})$ followed by acetone $(1 \mathrm{~mL})$. A few crystals $(<2 \mathrm{mg})$ of PPTS were added and the resulting solution allowed to stir at rt overnight. The reaction mixture was then concentrated and the crude oil was purified by column chromatography (5\% EtOAc/Hexanes) to provide (-)-SI9 (0.149 g, $98 \%$ yield) as a clear, colorless oil. IR (neat) $3048,2930,2856,1472,1379,1250,1103,1039 \mathrm{~cm}^{-1} ;[\alpha]_{D}{ }^{20}$ $-4.7\left(c\right.$ 0.27, $\left.\mathrm{CHCl}_{3}\right) ;{ }^{1} \mathrm{H}$ NMR $\left(500 \mathrm{MHz}, \mathrm{CDCl}_{3}\right) \delta$ 7.67-7.62 (m, $\left.4 \mathrm{H}\right), 7.42-7.31(\mathrm{~m}, 6 \mathrm{H}), 5.56(\mathrm{ddd}, J=$ 15.2, 6.6, $6.6 \mathrm{~Hz}, 1 \mathrm{H}), 5.40$ (dd, $J=15.2,7.7 \mathrm{~Hz}, 1 \mathrm{H}), 4.65(\mathrm{br} \mathrm{s}, 2 \mathrm{H}), 4.50$ (dd, J = 7.1, $7.1 \mathrm{~Hz}, 1 \mathrm{H})$, 3.91-3.82 (m, 2 H), 3.78-3.61 (m, 4 H), 3.48 (app t, J = 6.4 Hz, 2 H), $3.32(\mathrm{~s}, 3 \mathrm{H})$, 1.98-1.87 (m, $2 \mathrm{H})$, 1.81-1.43 (m, $7 \mathrm{H}), 1.41(\mathrm{~s}, 3 \mathrm{H}), 1.32(\mathrm{~s}, 3 \mathrm{H}), 1.02(\mathrm{~s}, 9 \mathrm{H}), 0.89(\mathrm{~d}, J=7.0 \mathrm{~Hz}, 3 \mathrm{H}), 0.88(\mathrm{~s}, 9 \mathrm{H}), 0.00$ (s, $6 \mathrm{H}) ;{ }^{13} \mathrm{C}$ NMR $\left(125 \mathrm{MHz}, \mathrm{CDCl}_{3}\right) \delta 139.0,137.4,132.7,130.6,128.6,127.6,110.0,95.7,80.2,79.0$, $75.0,71.1,71.0,61.7,60.6,54.0,33.6,33.5,29.6,25.8,25.7,24.8,23.9,22.9,22.4,15.7,14.8,7.1$, - 
10.1 ; high resolution mass spectrum (ES+) $\mathrm{m} / \mathrm{z} 763.4412\left[(\mathrm{M}+\mathrm{Na})^{+}\right.$; calcd for $\left.\mathrm{C}_{42} \mathrm{H}_{68} \mathrm{O}_{7} \mathrm{Si}_{2} \mathrm{Na}: 763.4504\right]$.

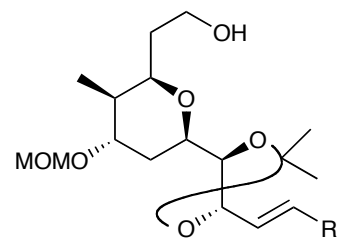

$(+)-23, \mathrm{R}=\left(\mathrm{CH}_{2}\right)_{3} \mathrm{OTBS}$

Alcohol (+)-23. To a flask containing (-)-S19 (0.022 g, $0.029 \mathrm{mmol}, 1.0$ equiv.) was added DMPU $(2 \mathrm{~mL})$ followed by $\mathrm{KOH}(0.25 \mathrm{~mL}, 8.0 \mathrm{M}$ aq. solution) and acetonitrile $(2 \mathrm{~mL})$ was then added. The resulting mixture was stirred at $\mathrm{rt}$ for $8 \mathrm{~h}$ at which time it was diluted with ether $(50 \mathrm{~mL})$ and washed with $2 \times 25 \mathrm{~mL}$ of $10 \% \mathrm{HCl}$ solution. The organic phase was then dried $\left(\mathrm{MgSO}_{4}\right)$ and concentrated to yield a crude oil. Purification was accomplished using column chromatography (10\% to $40 \%$ EtOAc/Hexanes) to provide first recovered (-)-SI9 $(0.006 \mathrm{~g}, 27 \%$ yield) and next (+)-23 $(0.010 \mathrm{~g}, 68 \%$ yield, 93\% BORSM) as a yellow oil: IR (neat) 3488, 2929, 2856, 1734, 1670, 1471, 1380, 1255, 1099, $1039 \mathrm{~cm}^{-1} ;[\alpha]_{\mathrm{D}}{ }^{20}+8.8\left(c 0.35, \mathrm{CHCl}_{3}\right) ;{ }^{1} \mathrm{H} \mathrm{NMR}\left(500 \mathrm{MHz}, \mathrm{CDCl}_{3}\right) \delta 5.84(\mathrm{ddd}, J=15.2,6.6,6.6 \mathrm{~Hz}, 1 \mathrm{H})$, $5.56(\mathrm{dd}, J=15.2,8.1 \mathrm{~Hz}, 1 \mathrm{H}), 4.68(\mathrm{br} \mathrm{s}, 2 \mathrm{H}), 4.59$ (dd, $J=8.5,6.3 \mathrm{~Hz}, 1 \mathrm{H}), 4.00-3.96(\mathrm{~m}, 1 \mathrm{H}), 3.94-$ $3.90(m, 1 H), 3.86-3.80(m, 1 H), 3.74-3.66(m, 3 H), 3.64$ (app t, J=5.9 Hz, 2 H), 3.37 (s, 3 H), 2.15 (ddd, $J=8.5,8.5,8.5 \mathrm{~Hz}, 2 \mathrm{H}), 1.84-1.73(\mathrm{~m}, 2 \mathrm{H}), 1.72-1.55(\mathrm{~m}, 5 \mathrm{H}), 1.44(\mathrm{~s}, 3 \mathrm{H}), 1.44-1.43(\mathrm{~m}, 1 \mathrm{H})$, $1.34(\mathrm{~s}, 3 \mathrm{H}), 0.94(\mathrm{~d}, J=7.0 \mathrm{~Hz}, 3 \mathrm{H}), 0.89(\mathrm{~s}, 9 \mathrm{H}), 0.05(\mathrm{~s}, 6 \mathrm{H}) ;{ }^{13} \mathrm{C} \mathrm{NMR}\left(125 \mathrm{MHz}, \mathrm{CDCl}_{3}\right) \delta 135.3$, $125.3,108.0,94.7,79.7,79.0,74.9,73.8,71.2,62.8,61.0,55.3,36.5,35.1,31.9,28.8,28.3,27.7,25.9$, $25.3,18.3,11.2,-5.4$; high resolution mass spectrum $(\mathrm{ES}+) \mathrm{m} / \mathrm{z} 525,3214\left[(\mathrm{M}+\mathrm{Na})^{+}\right.$; calcd for $\left.\mathrm{C}_{26} \mathrm{H}_{50} \mathrm{O}_{7} \mathrm{SiNa}: 525.3326\right]$.

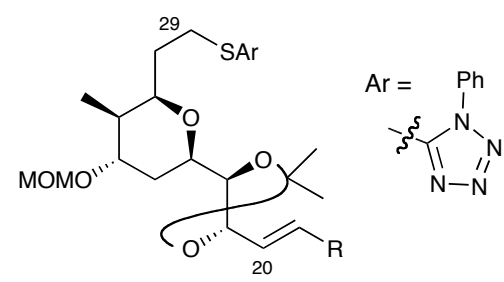

$(-)-\mathrm{SI10}, \mathrm{R}=\left(\mathrm{CH}_{2}\right)_{3} \mathrm{OTBS}$

Thioether (-)-SI10. To a flask containing (+)-23 $(0.035 \mathrm{~g}, 0.071 \mathrm{mmol}, 1.0$ equiv.) was added THF (1 mL). Triphenylphosphine (0.022 g, 1.2 equiv.), 1-phenyl-1H-tetrazole-5-thiol (0.025 g, 2.0 equiv.), 
and diisopropylazadicarboxylate $(0.02 \mathrm{~mL}, 1.4$ equiv.) were sequentially added and the resulting yellow solution allowed to mix at rt for $20 \mathrm{~min}$ at which point TLC analysis showed consumption of starting material. The reaction was concentrated to approximately $0.5 \mathrm{~mL}$ and loaded on a 1000 micron prep plate using 20\% EtOAc/Hexanes as eluent to provide $0.045 \mathrm{~g}$ (98\% yield) of (-)-SI10 as a clear, colorless oil: IR (neat) 2924, 2854, 1734, 1598, 1500, 1380, 1256, 1098, $1064 \mathrm{~cm}^{-1} ;[\alpha]_{\mathrm{D}}{ }^{20}-1.8\left(c 0.15, \mathrm{CHCl}_{3}\right) ;{ }^{1} \mathrm{H}$ NMR (500 MHz, $\left.\mathrm{CDCl}_{3}\right) \delta$ 7.61-7.52 (m, $5 \mathrm{H}$ ), 5.79 (ddd, $\left.J=15.6,6.7,6.7 \mathrm{~Hz}, 1 \mathrm{H}\right), 5.53(\mathrm{dd}, J=15.6,8.1$ $\mathrm{Hz}, 1 \mathrm{H}), 4.67(\mathrm{br} \mathrm{s}, 2 \mathrm{H}), 4.61(\mathrm{dd}, J=7.4,6.3 \mathrm{~Hz}, 1 \mathrm{H}), 3.93(\mathrm{dd}, J=8.1,6.3 \mathrm{~Hz}, 1 \mathrm{H}), 3.90$ (ddd, $J=$ 9.3, 2.9, $2.9 \mathrm{~Hz}, 1 \mathrm{H}), 3.80-3.73(\mathrm{~m}, 2 \mathrm{H}), 3.57$ (app t, J = 6.3 Hz, $2 \mathrm{H}), 3.35(\mathrm{~s}, 3 \mathrm{H}), 2.14-2.06(\mathrm{~m}, 3 \mathrm{H})$, 2.00-1.84 (m, 2 H), 1.83-1.76 (m, 2 H), 1.75-1.54 (m, 4 H), $1.43(\mathrm{~s}, 3 \mathrm{H}), 1.34$ (s, 3 H), 0.90 (d, J = 7.0 Hz, $3 \mathrm{H}), 0.85$ (s, $9 \mathrm{H}), 0.00$ (s, $6 \mathrm{H}) ;{ }^{13} \mathrm{C} \mathrm{NMR}\left(125 \mathrm{MHz}, \mathrm{CDCl}_{3}\right) \delta$ 154.2, 135.1, 133.7, 130.0, 129.7, 125.5, 123.7, $108.1,94.6,79.9,79.0,74.8,72.9,71.3,62.5,55.3,36.2,32.8,32.2,30.3,28.8,28.4,27.7,25.9$, $25.4,18.2,11.1,-5.4$ : high resolution mass spectrum $(\mathrm{ES}+) \mathrm{m} / \mathrm{z} 685.3427\left[(\mathrm{M}+\mathrm{Na})^{+}\right.$; calcd for $\mathrm{C}_{33} \mathrm{H}_{54} \mathrm{~N}_{4} \mathrm{O}_{6} \mathrm{SSiNa}:$ 685.3533].

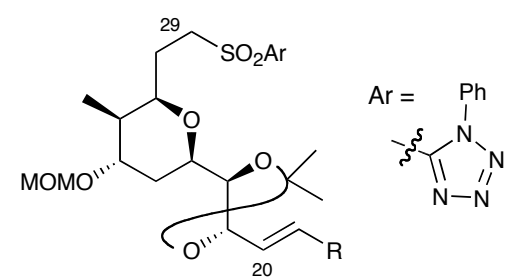

$(-)-2, \mathrm{R}=\left(\mathrm{CH}_{2}\right)_{3} \mathrm{OTBS}$

Sulfone (-)-2. To a flask containing (-)-S110 (0.029 g, 0.044 mmol, 1.0 equiv.) was added EtOH (3.2 $\mathrm{mL})$. The reaction flask was cooled to $0{ }^{\circ} \mathrm{C}$ and a pre-mixed solution of ammonium molybdate tetrahydrate $\left(0.021 \mathrm{~g}, 0.40\right.$ equiv.) and $\mathrm{H}_{2} \mathrm{O}_{2}(0.15 \mathrm{~mL})$ was then added dropwise. The flask containing the pre-mixed solution was then washed with $\mathrm{EtOH}(1 \mathrm{~mL})$ and this solution was added to the reaction flask. The resulting solution was then allowed to mix overnight slowly warming to rt. In the morning, the flask was diluted with $50 \mathrm{~mL}$ of ether and washed with $20 \mathrm{~mL}$ of sat. $\mathrm{NaHCO}_{3}$. The aqueous phase was then washed with $10 \mathrm{~mL}$ of ether and the combined organics were dried $\left(\mathrm{MgSO}_{4}\right)$ and concentrated to yield a crude oil. Purification was accomplished using a 1000 micron prep-TLC plate with an eluent of 20\% EtOAc/Hexanes to yield (-)-2 (0.029 g, 95\% yield) as a clear, colorless oil: IR (neat) 2930, 2857, 1782, 1596, 1498, 1380, 1344, 1250, 1216, 1152, 1099, $1037 \mathrm{~cm}^{-1} ;[\alpha]_{\mathrm{D}}{ }^{20}-1.6\left(c 1.05, \mathrm{CHCl}_{3}\right) ;{ }^{1} \mathrm{H} \mathrm{NMR}$ 
$\left(500 \mathrm{MHz}, \mathrm{CDCl}_{3}\right) \delta$ 7.72-7.55 (m, $\left.5 \mathrm{H}\right), 5.82(\mathrm{ddd}, J=15.2,6.7,6.7 \mathrm{~Hz}, 1 \mathrm{H}), 5.52(\mathrm{dd}, J=15.2,8.1 \mathrm{~Hz}, 1$ H), $4.69\left(\mathrm{~d}, J_{\mathrm{AB}}=7.0 \mathrm{~Hz}, 1 \mathrm{H}\right), 4.67\left(\mathrm{~d}, J_{\mathrm{AB}}=7.0 \mathrm{~Hz}, 1 \mathrm{H}\right), 4.61(\mathrm{dd}, J=8.9,6.3 \mathrm{~Hz}, 1 \mathrm{H}), 3.96(\mathrm{dd}, J=$ 7.4, $6.3 \mathrm{~Hz}, 1 \mathrm{H}), 3.90-3.81(\mathrm{~m}, 2 \mathrm{H}), 3.80-3.66(\mathrm{~m}, 3 \mathrm{H}), 3.61$ (app t, $J=6.7 \mathrm{~Hz}, 2 \mathrm{H}), 3.38$ (s, $3 \mathrm{H}), 2.17-$ $2.05(\mathrm{~m}, 3 \mathrm{H}), 1.92-1.54(\mathrm{~m}, 6 \mathrm{H}), 1.44(\mathrm{~s}, 3 \mathrm{H}), 1.34(\mathrm{~s}, 3 \mathrm{H}), 0.93(\mathrm{~d}, J=7.0 \mathrm{~Hz}, 3 \mathrm{H}), 0.87(\mathrm{~s}, 9 \mathrm{H}), 0.03$ (s, $6 \mathrm{H}) ;{ }^{13} \mathrm{C}$ NMR $\left(125 \mathrm{MHz}, \mathrm{CDCl}_{3}\right) \delta 153.3,135.3,133.0,131.4,129.7,125.5,125.0,108.2,94.7,79.7$, $79.0,74.7,72.5,71.4,62.5,55.4,54.0,36.3,32.1,28.7,28.2,27.7,26.0,25.9,25.3,18.3,10.9$, -5.3; high resolution mass spectrum (ES+) $m / 27173304$ [(M+Na) ${ }^{+}$; calcd for $\left.\mathrm{C}_{33} \mathrm{H}_{54} \mathrm{~N}_{4} \mathrm{O}_{8} \mathrm{SiNa}: 717.3432\right]$.

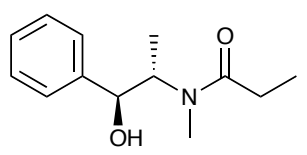

$(+)-24$

Amide (+)-24. A solution of (1S,2S)-(+)-pseudoephedrine (21.54 g, $130.4 \mathrm{mmole})$ in $\mathrm{CH}_{2} \mathrm{Cl}_{2}(250$ $\mathrm{mL}$ ) was placed in an ambient temperature water bath and charged with $\mathrm{Et}_{3} \mathrm{~N}(22.0 \mathrm{~mL}, 1.20$ equiv.). Propionic anhydride (17.9 mL, 1.07 equiv.) was then added in $1.0 \mathrm{~mL}$ aliquots over $5 \mathrm{~min}$., and after 30 min., water $(40 \mathrm{~mL})$ was added. The organic layer was then washed with $50 \%$ sat. $\mathrm{NaHCO}_{3}(2 \times 40 \mathrm{~mL}), 1$ $\mathrm{N} \mathrm{HCl}(2 \times 40 \mathrm{~mL})$, dried over $\mathrm{MgSO}_{4}$ and concentrated in vacuo. The solid was then recrystallized from hot toluene $(85 \mathrm{~mL})$ to afford $26.82 \mathrm{~g}\left(93 \%\right.$ yield) of amide $(+)-24$ as a white solid: $\mathrm{mp} 114-115{ }^{\circ} \mathrm{C} ; \mathrm{R}_{\mathrm{f}} 0.40$ (5/1, hexanes/EtOAc); $[\alpha]_{D}^{20}+103.1(c 0.66, \mathrm{MeOH}) ; \mathrm{IR}$ (neat) $3379(\mathrm{br}, \mathrm{m}), 2979(\mathrm{~m}), 2937(\mathrm{~m}), 1623(\mathrm{~s})$, $1053(\mathrm{~s}) \mathrm{cm}^{-1} ;{ }^{1} \mathrm{H}$ NMR $\left(500 \mathrm{MHz}, \mathrm{C}_{6} \mathrm{D}_{6}, 3: 1\right.$ mixture of rotamers, "represents minor peaks) $\delta 7.31(\mathrm{~d}, \mathrm{~J}=$ 7.3 Hz, $2 \mathrm{H}), 7.11(\mathrm{~m}, 3 \mathrm{H}), 4.77(\mathrm{br} \mathrm{s}, 1 \mathrm{H}), 4.51(\mathrm{~d}, \mathrm{~J}=7.3 \mathrm{~Hz}, 1 \mathrm{H}), 4.20(\mathrm{~m}, 1 \mathrm{H}),{ }^{\star} 3.70(\mathrm{~m}, 1 \mathrm{H}),{ }^{*} 2.80$ (s, $3 \mathrm{H}),{ }^{\star} 2.43(\mathrm{~m}, 1 \mathrm{H}), 2.12(\mathrm{~s}, 3 \mathrm{H}), 1.76(\mathrm{~m}, 2 \mathrm{H}),{ }^{*} 1.21$ (app t, J= 7.3 Hz, $\left.3 \mathrm{H}\right), 1.01$ (app t, J= $7.4 \mathrm{~Hz}, 3$ H), 0.95 (d, J=7.0 Hz, $3 \mathrm{H}),{ }^{\star} 0.57$ (d, J=6.7 Hz, $\left.3 \mathrm{H}\right) ;{ }^{13} \mathrm{C}$ NMR $\left(125 \mathrm{MHz}, \mathrm{CDCl}_{3}\right) \delta 175.9,{ }^{\star} 174.8,142.3$, *141.4, *128.5, 128.2, * $128.1,127.4,{ }^{*} 126.8,126.3,76.3,{ }^{*} 75.2,58.1,32.3,27.4,{ }^{*} 26.6,{ }^{*} 15.2,14.3,{ }^{*} 9.5$, 9.0; high resolution mass spectrum $\left(\mathrm{Cl}, \mathrm{NH}_{3}\right) \mathrm{m} / \mathrm{z} 222.1486$ [ $\mathrm{MH}^{+}$; calcd for $\mathrm{C}_{13} \mathrm{H}_{20} \mathrm{NO}_{2}$ : 222.1493].

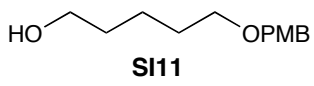


Alcohol Sl11. A $0{ }^{\circ} \mathrm{C}$ suspension of $\mathrm{NaH}(26.62 \mathrm{~g}, 2.2$ equiv., $95 \%$ wt.) in THF (1.5 L) was charged with a solution of distilled 1,5-pentanediol (336.19 g, 6.7 equiv.) in THF (500 mL) via cannula over $15 \mathrm{~min}$. Gas Evolution! The suspension was then mechanical stirred, heated to reflux, and after $3 \mathrm{~h}$, sequentially charged with tetrabutylammonium iodide $(8.85 \mathrm{~g}, 0.05$ equiv.) and $p$-methoxybenzyl chloride (64.9 $\mathrm{mL}, 478 \mathrm{mmole})$. After $15 \mathrm{~h}$, the reaction was quenched with water $(500 \mathrm{~mL})$ and concentrated in vacuo. The residue was then diluted with $\mathrm{CH}_{2} \mathrm{Cl}_{2}(1.0 \mathrm{~L})$ and water $(2.5 \mathrm{~L})$. The aqueous phase was then washed with $\mathrm{CH}_{2} \mathrm{Cl}_{2}(3 \times 250 \mathrm{~mL})$, and the combined organic layers were then washed with water $(3 \times 500$ $\mathrm{mL}$ ), dried over $\mathrm{MgSO}_{4}$ and concentrated in vacuo to afford a yellow oil. Purification by flash chromatography $\left(10 / 1 \rightarrow 3 / 2\right.$, hexanes/EtOAc) afforded $106.20 \mathrm{~g}$ (99\% yield) of Sl11 as a yellow oil: $\mathrm{R}_{\mathrm{f}}$ 0.17 (2/1 hexanes/EtOAc); IR (neat) 3398 (br, m), 2936 (s), 2861 (s), 1612 (s), 1585 (w), 1512 (s), 1248 (s), $1173(\mathrm{~m}), 1094$ (s), 1035 (s) cm ${ }^{-1}$; ${ }^{1} \mathrm{H}$ NMR $\left(500 \mathrm{MHz}, \mathrm{CDCl}_{3}\right) \delta 7.25$ (d, J=8.6 Hz, $2 \mathrm{H}$ ), 6.87 (d, J= $8.6 \mathrm{~Hz}, 2 \mathrm{H}), 4.43(\mathrm{~s}, 2 \mathrm{H}), 3.80(\mathrm{~s}, 3 \mathrm{H}), 3.63(\mathrm{t}, \mathrm{J}=6.5 \mathrm{~Hz}, 2 \mathrm{H}), 3.45(\mathrm{t}, \mathrm{J}=6.5 \mathrm{~Hz}, 2 \mathrm{H}), 1.60(\mathrm{~m}, 4 \mathrm{H})$, $1.45(\mathrm{~m}, 2 \mathrm{H}) ;{ }^{13} \mathrm{C}$ NMR $\left(125 \mathrm{MHz}, \mathrm{CDCl}_{3}\right) \delta 158.9,130.4,129.0,113.6,72.3,69.8,62.3,55.0,32.2,29.2$, 22.2; high resolution mass spectrum $\left(\mathrm{Cl}, \mathrm{NH}_{3}\right) \mathrm{m} / \mathrm{z} 224.1412\left[\mathrm{M}^{+}\right.$; calcd for $\left.\mathrm{C}_{13} \mathrm{H}_{20} \mathrm{O}_{3}: 224.1412\right]$.

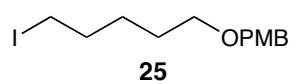

lodide 25. To a $0{ }^{\circ} \mathrm{C}$ solution of alcohol Sl11 (18.10 g, 80.7 mmole) and imidazole $(13.19 \mathrm{~g}, 2.4$ equiv.) in THF (340 mL), wrapped in Al foil, was sequentially added triphenylphosphine (25.42 g, 1.2 equiv.) and THF (30 mL). After $10 \mathrm{~min}$, iodine (24.57 g, 1.2 equiv.) was added portionwise over $5 \mathrm{~min}$ followed by additional THF (30 mL). After $15 \mathrm{~min}$, the reaction was concentrated in vacuo. The resulting slurry was then diluted with EtOAc $(300 \mathrm{~mL})$, and washed with sat. $\mathrm{Na}_{2} \mathrm{~S}_{2} \mathrm{O}_{3}(2 \times 100 \mathrm{~mL})$, sat. $\mathrm{NaHCO}_{3}(2$ x $100 \mathrm{~mL}$ ), dried over $\mathrm{MgSO}_{4}$ and concentrated in vacuo. The resulting yellow solid was the preabsorbed onto $200 \mathrm{~mL}$ Si gel. Purification by flash chromatography (20/1 $\rightarrow 2 / 1$, hexanes/EtOAc) afforded $24.96 \mathrm{~g}$ (93\% yield) of 25 as a yellow oil: $R_{f} 0.33$ (10/1 hexanes/EtOAc); IR (neat) 2934 (s), 2856 (s), 1612 (s), 1585 (m), 1512 (s), 1456 (s), 1360 (m), 1301 (s), 1247 (s), 1171 (s), 1099 (s), 1035 (s) cm ${ }^{-1}$; ${ }^{1} \mathrm{H}$ NMR (500 $\left.\mathrm{MHz}, \mathrm{CDCl}_{3}\right) \delta 7.25$ (d, J=8.6 Hz, $2 \mathrm{H}$ ), 6.88 (d, J=8.6 Hz, $2 \mathrm{H}$ ), 4.43 (s, $2 \mathrm{H}$ ), 3.81 (s, $\left.3 \mathrm{H}\right), 3.44$ (t, J= 6.5 $\mathrm{Hz}, 2 \mathrm{H}), 3.18(\mathrm{t}, \mathrm{J}=7.0 \mathrm{~Hz}, 2 \mathrm{H}), 1.84(\mathrm{~m}, 2 \mathrm{H}), 1.62(\mathrm{~m}, 2 \mathrm{H}), 1.48(\mathrm{~m}, 2 \mathrm{H}) ;{ }^{13} \mathrm{C} \mathrm{NMR}\left(125 \mathrm{MHz}, \mathrm{CDCl}_{3}\right)$ 
$\delta 159.0,130.5,129.0,113.6,72.4,69.5,55.1,33.2,28.5,27.1,6.7$; high resolution mass spectrum $(\mathrm{Cl}$, $\left.\mathrm{NH}_{3}\right) \mathrm{m} / \mathrm{z} 335.0508\left[\mathrm{MH}^{+}\right.$; calcd for $\left.\mathrm{C}_{13} \mathrm{H}_{20} \mathrm{IO}_{2}: 335.0507\right]$.

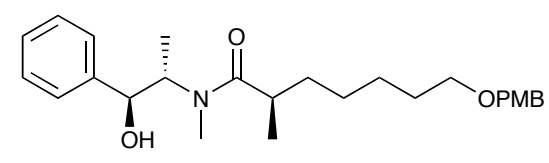

$(+)-26$

Amide (+)-26. LiCl (9.64 g, 7.6 equiv.) was first flame-dried under high vacuum and cooled under Ar. THF (88 mL), and $(i-\operatorname{Pr})_{2} \mathrm{NH}(18.0 \mathrm{~mL}, 4.3$ equiv.) were then added sequentially and the suspension was cooled to $-78{ }^{\circ} \mathrm{C}$. $n$-BuLi $(47.8 \mathrm{~mL}, 2.5 \mathrm{M}$ in hexanes, 4.0 equiv.) was then added via syringe over 3$4 \mathrm{~min}$, followed by a $0{ }^{\circ} \mathrm{C}$ solution of amide (+)-24 (13.89 g, 2.1 equiv.) in THF (178 mL) via cannula. After an additional $1 \mathrm{~h}$, the suspension was warmed to $0{ }^{\circ} \mathrm{C}$ for $15 \mathrm{~min}$, and then to ambient temperature for 5 $\min$. The reaction was then re-cooled to $0{ }^{\circ} \mathrm{C}$, and charged with a solution of azeotroped iodide 25 (10.02 g, 29.9 mmole) in THF (28 mL). After an additional $2.5 \mathrm{~h}$, the reaction was carefully quenched with sat. $\mathrm{NH}_{4} \mathrm{Cl}(60 \mathrm{~mL})$ and concentrated in vacuo. EtOAc $(185 \mathrm{~mL})$ and water $(185 \mathrm{~mL})$ were then added, and the aqueous layer was washed with EtOAc $(2 \times 40 \mathrm{~mL})$. The combined organic layers were then dried over $\mathrm{MgSO}_{4}$, concentrated in vacuo and preabsorbed onto $90 \mathrm{~mL} \mathrm{Si} \mathrm{gel.} \mathrm{Purification} \mathrm{by} \mathrm{flash}$ chromatography $(1 / 3$, hexanes/EtOAc $\rightarrow$ EtOAc) afforded $12.63 \mathrm{~g}(99 \%$ yield) of $(+)-26$ as a yellow oil and $6.24 \mathrm{~g}(86 \%)$ of recovered $(+)-24$. For $(+)-26: R_{\mathrm{f}} 0.56\left(1 / 5\right.$ hexanes/EtOAc); $[\alpha]_{\mathrm{D}}^{20}+49.1(c 0.77$, $\mathrm{CHCl}_{3}$ ); IR (neat) 3388 (br, s), 2933 (s), 2856 (s), 1616 (s), 1513 (s), 1464 (m), 1301 (m), 1247 (s), 1098 (s), 1035 (s) $\mathrm{cm}^{-1} ;{ }^{1} \mathrm{H}$ NMR $\left(500 \mathrm{MHz}, \mathrm{CDCl}_{3}, 5: 1\right.$ mixture of rotamers, ${ }^{*}$ represents minor peaks) $\delta 7.24$ (m, $5 \mathrm{H}), 7.15(\mathrm{~d}, J=8.6 \mathrm{~Hz}, 2 \mathrm{H}), 6.77(\mathrm{~d}, J=8.6 \mathrm{~Hz}, 2 \mathrm{H}), 4.51(\mathrm{dd}, J=7.2,7.2 \mathrm{~Hz}, 1 \mathrm{H}), 4.43(\mathrm{~m}, 1 \mathrm{H})$, $4.32(\mathrm{br} \mathrm{s}, 2 \mathrm{H}),{ }^{\star} 3.98(\mathrm{~m}, 1 \mathrm{H}), 3.69(\mathrm{~s}, 3 \mathrm{H}), 3.32(\operatorname{app~t}, \mathrm{J}=6.5 \mathrm{~Hz}, 2 \mathrm{H}),{ }^{*} 2.79(\mathrm{~s}, 3 \mathrm{H}), 2.73(\mathrm{~s}, 3 \mathrm{H})$, $2.47(\mathrm{~m}, 1 \mathrm{H}), 2.13(\mathrm{~d}, J=1.7 \mathrm{~Hz}, 1 \mathrm{H}),{ }^{*} 1.72(\mathrm{~m}, 1 \mathrm{H}), 1.48(\mathrm{~m}, 2 \mathrm{H}), 1.00-1.30(\mathrm{~m}, 6 \mathrm{H}), 1.03(\mathrm{~d}, J=6.9$ $\mathrm{Hz}, 3 \mathrm{H}), 0.97(\mathrm{~d}, \mathrm{~J}=6.7 \mathrm{~Hz}, 3 \mathrm{H}),{ }^{*} 0.90(\mathrm{~d}, \mathrm{~J}=6.7 \mathrm{~Hz}, 3 \mathrm{H}) ;{ }^{13} \mathrm{C} \mathrm{NMR}\left(125 \mathrm{MHz}, \mathrm{CDCl}_{3}\right) \delta 178.5,{ }^{*} 177.5$, $159.0,142.6,{ }^{*} 141.9,130.7,129.1,{ }^{*} 128.4,{ }^{*} 128.2,128.1,{ }^{*} 127.9,127.3,{ }^{*} 126.8,126.2,113.6,76.0,75.3$, $72.3,{ }^{\star} 70.1,69.9,57.6,55.1,36.3,{ }^{*} 35.5,33.8,{ }^{\star} 29.6,29.5,{ }^{\star} 27.2,27.1,{ }^{*} 26.2,26.1,{ }^{*} 18.0,17.2,{ }^{*} 15.5$, 14.3; high resolution mass spectrum (ES+) $\mathrm{m} / \mathrm{z} 450.2626\left[(\mathrm{M}+\mathrm{Na})^{+}\right.$; calcd for $\left.\mathrm{C}_{26} \mathrm{H}_{37} \mathrm{NO}_{4} \mathrm{Na}^{+}: 450.2619\right]$. 


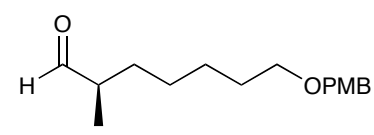

$(-)$-SI12

Aldehyde (-)-Sl12. A $0{ }^{\circ} \mathrm{C}$ suspension of lithium aluminum hydride (937 mg, 2.30 equiv., $\left.95 \%\right)$ and HPLC hexanes $(54 \mathrm{~mL})$ was charged with HPLC EtOAc $(3.4 \mathrm{~mL})$ over $10 \mathrm{~min}$, and cooled to $-78{ }^{\circ} \mathrm{C}$. A solution of azeotroped amide (+)-26 (4.37 g, $10.2 \mathrm{mmole})$ in THF (34 mL) was then added via cannula. Gas Evolution! The reaction was then warmed to $0{ }^{\circ} \mathrm{C}$, and after $1.5 \mathrm{~h}$, cannulated into a solution of $1 \mathrm{~N}$ $\mathrm{HCl}(125 \mathrm{~mL})$ and trifluoroacetic acid $(7.8 \mathrm{~mL})$, and rinsed with THF $(3 \times 5 \mathrm{~mL})$. After $10 \mathrm{~min}$, the biphasic mixture was diluted with $1 \mathrm{~N} \mathrm{HCl}(220 \mathrm{~mL})$, and the aqueous layer was washed with $7 / 1$ hexanes/EtOAc (3 $x 50 \mathrm{~mL})$. The combined organic layers were then neutralized by the careful addition of sat. $\mathrm{NaHCO}_{3}(90$ $\mathrm{mL}$ ), dried over $\mathrm{MgSO}_{4}$, filtered through a plug of Si gel supported by celite (rinsing with 7/1 hexanes/EtOAc), and concentrated in vacuo to afford a yellow oil. Purification by flash chromatography $\left(7 / 1 \rightarrow 2 / 1\right.$, hexanes/EtOAc) afforded $2.07 \mathrm{~g}\left(77 \%\right.$ yield) of $(-)$-SI12 as a pale yellow oil: $R_{\mathrm{f}} 0.33(4 / 1$ hexanes/EtOAc); $[\alpha]_{\mathrm{D}}^{20}-14.8\left(c 0.53, \mathrm{CHCl}_{3}\right) ; \mathrm{IR}$ (neat) $2934(\mathrm{~s}), 2857(\mathrm{~s}), 2709(\mathrm{w}), 1724(\mathrm{~s}), 1612(\mathrm{~m})$, 1585 (w), 1511 (s), 1464 (s), 1362 (m), 1301 (m), 1247 (s), 1172 (m), 1097 (s), 1035 (s) cm ${ }^{-1} ;{ }^{1} \mathrm{H}^{\mathrm{N} M R}$ $\left(500 \mathrm{MHz}, \mathrm{CDCl}_{3}\right) \delta 9.60(\mathrm{~d}, J=2.0 \mathrm{~Hz}, 1 \mathrm{H}), 7.25(\mathrm{~d}, J=8.6 \mathrm{~Hz}, 2 \mathrm{H}), 6.87(\mathrm{~d}, J=8.6 \mathrm{~Hz}, 2 \mathrm{H}), 4.42(\mathrm{br}$, $2 \mathrm{H}), 3.80(\mathrm{~s}, 3 \mathrm{H}), 3.43(\operatorname{app~t}, \mathrm{J}=6.5 \mathrm{~Hz}, 2 \mathrm{H}), 2.31(\mathrm{~m}, 1 \mathrm{H}), 1.70(\mathrm{~m}, 2 \mathrm{H}), 1.60(\mathrm{~m}, 2 \mathrm{H}), 1.35(\mathrm{~m}, 4 \mathrm{H})$, $1.08(\mathrm{~d}, J=7.0 \mathrm{~Hz}, 3 \mathrm{H}) ;{ }^{13} \mathrm{C} \mathrm{NMR}\left(125 \mathrm{MHz}, \mathrm{CDCl}_{3}\right) \delta 205.1,159.1,130.7,129.1,113.7,72.5,69.8,55.2$, 46.2, 30.4, 29.6, 26.7, 26.2, 13.2; high resolution mass spectrum $\left(\mathrm{Cl}, \mathrm{NH}_{3}\right) \mathrm{m} / \mathrm{z} 264.1732\left[\mathrm{M}^{+}\right.$; calcd for $\left.\mathrm{C}_{16} \mathrm{H}_{24} \mathrm{O}_{3}{ }^{+}: 264.1725\right]$.

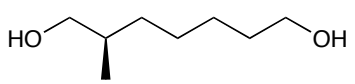

(+)-SI13

Diol (+)-SI13. To a $0{ }^{\circ} \mathrm{C}$ solution of aldehyde (-)-SI12 $(79.0 \mathrm{mg}, 0.299 \mathrm{mmole})$ in absolute EtOH $(1.0 \mathrm{~mL})$ was added sodium borohydride $(22.6 \mathrm{mg}, 2.0$ equiv.). After $20 \mathrm{~min}$, the reaction was diluted with sat. $\mathrm{NH}_{4} \mathrm{Cl}(10 \mathrm{~mL})$ and $\mathrm{CH}_{2} \mathrm{Cl}_{2}(10 \mathrm{~mL})$. The aqueous layer was then washed with $\mathrm{CH}_{2} \mathrm{Cl}_{2}(2 \times 3 \mathrm{~mL})$, and the combined organic layers were dried over $\mathrm{MgSO}_{4}$ and concentrated in vacuo to afford $79.2 \mathrm{mg}$ of the alcohol that was used without further purification. To a $0{ }^{\circ} \mathrm{C}$ solution of the above alcohol $(79.2 \mathrm{mg}, 297$ 
mmole) in $\mathrm{CH}_{3} \mathrm{CN}(7.7 \mathrm{~mL})$ and $\mathrm{pH} 7$ buffer $(770 \mu \mathrm{L})$ was added ceric ammonium nitrate (489 $\mathrm{mg}, 3.0$ equiv.) in one portion. The reaction was then allowed to warm to ambient temperature, and after $1 \mathrm{~h} 15$ min, was diluted with $\mathrm{CH}_{2} \mathrm{Cl}_{2}(20 \mathrm{~mL})$, sat. $\mathrm{NaHCO}_{3}(10 \mathrm{~mL})$ and water $(20 \mathrm{~mL})$. The aqueous layer was then washed with $\mathrm{CH}_{2} \mathrm{Cl}_{2}(4 \times 5 \mathrm{~mL})$, and the combined organic layers were dried over $\mathrm{Na}_{2} \mathrm{SO}_{4}$ and concentrated in vacuo to afford a pale yellow oil. Purification by flash chromatography $(1 / 1 \rightarrow 2 / 3$, hexanes/EtOAc) afforded $37 \mathrm{mg}\left(85 \%\right.$ yield, 2 steps) of (+)-SI13 as a pale yellow oil: $R_{f} 0.15(1 / 1$ hexanes/EtOAc); $[\alpha]_{\mathrm{D}}^{20}+9.8\left(c 0.56, \mathrm{CH}_{2} \mathrm{Cl}_{2}\right) ; \mathrm{IR}$ (thin film, $\left.\mathrm{CDCl}_{3}\right) 3324$ (br, s), 2929 (s), 2857 (s), 1463

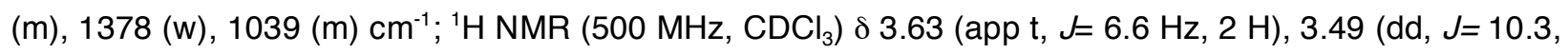
$5.9 \mathrm{~Hz}, 1 \mathrm{H}), 3.42(\mathrm{dd}, J=10.3,6.5 \mathrm{~Hz}, 1 \mathrm{H}), 1.58(\mathrm{~m}, 3 \mathrm{H}), 1.34(\mathrm{~m}, 7 \mathrm{H}), 1.12(\mathrm{~m}, 1 \mathrm{H}), 0.91(\mathrm{~d}, J=6.7$ $\mathrm{Hz}, 3 \mathrm{H}) ;{ }^{13} \mathrm{C}$ NMR $\left(125 \mathrm{MHz}, \mathrm{CDCl}_{3}\right) \delta 69.2,62.9,35.6,33.0,32.6,26.6,25.9,16.4$; high resolution mass spectrum $\left(\mathrm{Cl}, \mathrm{NH}_{3}\right) \mathrm{m} / \mathrm{z} 147.1378\left[\mathrm{MH}^{+}\right.$; calcd for $\left.\mathrm{C}_{8} \mathrm{H}_{19} \mathrm{O}_{2}^{+}: 147.1385\right]$.

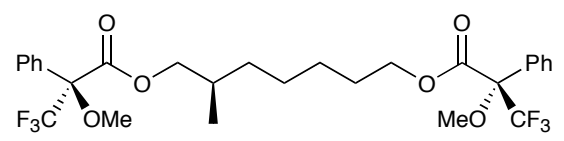

$(-)-$ S114

Bisester (-)-SI14. To the (S)-(-)- $\alpha$-methoxy- $\alpha$-trifluoromethyl-phenylacetic acid [(S)-MTPAOH, $72.0 \mathrm{mg}, \quad 3.0 \quad$ equiv.], $\quad 4-(\mathrm{N}, \mathrm{N}$-dimethylamino $)$ pyridine $\quad(112.7 \mathrm{mg}, \quad 9.0$ equiv. $)$ and 1 -[3(dimethylamino)propyl]3-ethyl-carbodiimide hydrochloride $\left(\mathrm{EDC}^{*} \mathrm{HCl}, 58.9 \mathrm{mg}, 3.0\right.$ equiv.) was added a solution of $(+)$-SI13 in $\mathrm{CH}_{2} \mathrm{Cl}_{2}(500 \mu \mathrm{L})$ at ambient temperature. After $2 \mathrm{~h}$, the solution was diluted with sat. $\mathrm{NH}_{4} \mathrm{Cl}(10 \mathrm{~mL})$ and $\mathrm{EtOAc}(10 \mathrm{~mL})$. The organic layer was then washed with sat. $\mathrm{NH}_{4} \mathrm{Cl}(10 \mathrm{~mL})$, sat. $\mathrm{NaHCO}_{3}(2 \times 5 \mathrm{~mL})$, dried over $\mathrm{MgSO}_{4}$ and concentrated in vacuo. Direct purification by Preparative-TLC (2/1, hexanes/EtOAc, $1000 \mu \mathrm{m}$ plate) afforded $5.1 \mathrm{mg}(9 \%$ yield, $>20: 1$ ratio of diastereomers) of the bis(S)-MTPA ester (-)-SI14 as a colorless oil which was identical in all respects to the characterization data previously reported in the literature. ${ }^{4}$ The remaining mass balance $(12.3 \mathrm{mg})$ consisted of a mixture $(\sim 1: 1)$ of monoesterified products. For $(-)-S \mid 14: R_{f} 0.88\left(1 / 1\right.$, hexanes/EtOAc); $[\alpha]_{D}^{20}-43.9(c) 0.25$, $\mathrm{CHCl}_{3}$ ); IR (thin film, $\mathrm{CH}_{2} \mathrm{Cl}_{2}$ ) 2945 (m), 2851 (w), 1747 (s), 1451 (w), 1272 (m), 1168 (s), 1122 (m), 1081 (w), $1022(\mathrm{~m}) \mathrm{cm}^{-1} ;{ }^{1} \mathrm{H}$ NMR (500 MHz, $\left.\mathrm{CDCl}_{3}\right) \delta 7.51(\mathrm{~m}, 4 \mathrm{H}), 7.39(\mathrm{~m}, 6 \mathrm{H}), 4.31(\mathrm{~m}, 1 \mathrm{H}), 4.26(\mathrm{~m}, 1 \mathrm{H})$, $4.16(\mathrm{ddd}, J=10.7,6.3,1.1 \mathrm{~Hz}, 1 \mathrm{H}), 4.11(\mathrm{ddd}, \mathrm{J}=10.7,5.7,1.1 \mathrm{~Hz}, 1 \mathrm{H}), 3.54(\mathrm{~s}, 6 \mathrm{H}), 1.80(\mathrm{~m}, 1 \mathrm{H})$, 
$1.65(\mathrm{~m}, 2 \mathrm{H}), 1.23-1.32(\mathrm{~m}, 5 \mathrm{H}), 1.13(\mathrm{~m}, 1 \mathrm{H}), 0.90(\mathrm{~d}, \mathrm{~J}=6.7 \mathrm{~Hz}, 3 \mathrm{H})$; high resolution mass spectrum $(\mathrm{ES}+) \mathrm{m} / \mathrm{z} 601.2027\left[(\mathrm{M}+\mathrm{Na})^{+}\right.$; calcd for $\mathrm{C}_{28} \mathrm{H}_{32} \mathrm{~F}_{6} \mathrm{O}_{6} \mathrm{Na:}$ 601.2103].

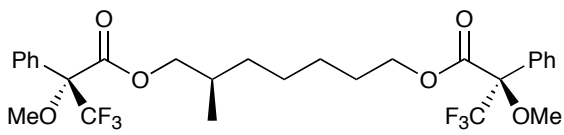

(+)-SI15

Bisester (+)-SI15. To the $(R)-(+)-\alpha-$ methoxy- $\alpha$-trifluoromethyl-phenylacetic acid $[(R)-\mathrm{MTPAOH}$, 72.0 mg, $3.0 \quad$ equiv.], $\quad 4-(N, N$-dimethylamino)pyridine $\quad(112.7 \quad \mathrm{mg}, \quad 9.0$ equiv. $)$ and 1 -[3(dimethylamino)propyl]3-ethyl-carbodiimide hydrochloride $\left(\mathrm{EDC}^{*} \mathrm{HCl}, 58.9 \mathrm{mg}, 3.0\right.$ equiv.) was added a solution of $(+)$-SI13 in $\mathrm{CH}_{2} \mathrm{Cl}_{2}(500 \mu \mathrm{L})$ at ambient temperature. After $2 \mathrm{~h}$, the solution was diluted with sat. $\mathrm{NH}_{4} \mathrm{Cl}(10 \mathrm{~mL})$ and $\mathrm{EtOAc}(10 \mathrm{~mL})$. The organic layer was then washed with sat. $\mathrm{NH}_{4} \mathrm{Cl}(10 \mathrm{~mL})$, sat. $\mathrm{NaHCO}_{3}(2 \times 5 \mathrm{~mL})$, dried over $\mathrm{MgSO}_{4}$ and concentrated in vacuo. Direct purification by Preparative-TLC (2/1, hexanes/EtOAc, $1000 \mu \mathrm{m}$ plate) afforded $7.6 \mathrm{mg}$ (13\% yield, $>20: 1$ ratio of diastereomers) of the bis(R)-MTPA ester (+)-SI15 as a colorless oil, which was identical in all respects to the characterization data previously reported in the literature. ${ }^{4}$ The remaining mass balance $(13.5 \mathrm{mg})$ consisted of a mixture $(\sim 1: 1)$ of monoesterified products. For $(+)-$ SI15: $R_{f} 0.88\left(1 / 1\right.$, hexanes/EtOAc); $[\alpha]_{D}^{20}+39.5(c 0.38$, $\mathrm{CHCl}_{3}$ ); IR (thin film, $\mathrm{CH}_{2} \mathrm{Cl}_{2}$ ) 2946 (m), 2852 (w), 1747 (s), 1451 (m), 1272 (s), 1169 (s), 1122 (m), 1081 (w), $1022(\mathrm{~m}) \mathrm{cm}^{-1} ;{ }^{1} \mathrm{H}$ NMR $\left(500 \mathrm{MHz}, \mathrm{CDCl}_{3}\right) \delta 7.51(\mathrm{~m}, 4 \mathrm{H}), 7.39(\mathrm{~m}, 6 \mathrm{H}), 4.32$ (ddd, J= 10.8, $6.6 \mathrm{~Hz}$, $1 \mathrm{H}), 4.27$ (ddd, J= 10.8, 6.6, 6.6 Hz, $1 \mathrm{H}$ ), 4.20 (dd, J=10.7, $5.8 \mathrm{~Hz}, 1 \mathrm{H}), 4.06$ (dd, J= 10.7, $6.5 \mathrm{~Hz}, 1 \mathrm{H}$ ), $3.54(\mathrm{~s}, 6 \mathrm{H}), 1.81(\mathrm{~m}, 1 \mathrm{H}), 1.66(\mathrm{~m}, 2 \mathrm{H}), 1.22-1.33(\mathrm{~m}, 5 \mathrm{H}), 1.13(\mathrm{~m}, 1 \mathrm{H}), 0.89(\mathrm{~d}, \mathrm{~J}=6.7 \mathrm{~Hz}, 3 \mathrm{H})$; high resolution mass spectrum (ES+) $\mathrm{m} / \mathrm{z} 601.1983\left[(\mathrm{M}+\mathrm{Na})^{+}\right.$; calcd for $\mathrm{C}_{28} \mathrm{H}_{32} \mathrm{~F}_{6} \mathrm{O}_{6} \mathrm{Na:}$ 601.2103].

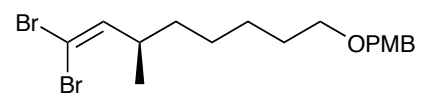

$(-)$-SI16

Gem-dibromide (-)-Sl16. To a $0{ }^{\circ} \mathrm{C}$ solution of triphenylphosphine (17.69 g, 4.0 equiv.) in $\mathrm{CH}_{2} \mathrm{Cl}_{2}(31 \mathrm{~mL})$ was added a solution of carbon tetrabromide $\left(11.18 \mathrm{~g}, 2.0\right.$ equiv.) in $\mathrm{CH}_{2} \mathrm{Cl}_{2}(30 \mathrm{~mL})$ via cannula over 1-2 min. After an additional $30 \mathrm{~min}$, the orange suspension was treated with a solution of aldehyde (-)-SI12 (4.46 g, 16.9 mmole) in $\mathrm{CH}_{2} \mathrm{Cl}_{2}(15 \mathrm{~mL})$ via cannula. After $1.5 \mathrm{~h}$, the reaction was 
triturated with pentane $(150 \mathrm{~mL})$, and the resulting suspension was filtered through a pad of Si gel, rinsing with hexanes/EtOAc (10:1), and concentrated in vacuo. Purification by flash chromatography (15/1 $\rightarrow$ 10/1, hexanes/EtOAc) afforded $6.72 \mathrm{~g}\left(95 \%\right.$ yield) of (-)-SI16 as a pale yellow oil: $R_{f} 0.34(10 / 1$ hexanes/EtOAc); $[\alpha]_{\mathrm{D}}^{20}-5.6\left(c 0.51, \mathrm{CHCl}_{3}\right)$; IR (neat) $2931(\mathrm{~s}), 2852(\mathrm{~s}), 1612(\mathrm{~m}), 1511(\mathrm{~s}), 1464(\mathrm{~m})$, 1362 (w), 1301 (m), 1247 (s), 1172 (m), 1097 (s), 1037 (s) cm ${ }^{-1} ;{ }^{1} \mathrm{H}$ NMR $\left(500 \mathrm{MHz}, \mathrm{CDCl}_{3}\right) \delta 7.26$ (d, J= $8.5 \mathrm{~Hz}, 2 \mathrm{H}), 6.87(\mathrm{~d}, J=8.5 \mathrm{~Hz}, 2 \mathrm{H}), 6.15(\mathrm{~d}, \mathrm{~J}=9.4 \mathrm{~Hz}, 1 \mathrm{H}), 4.43(\mathrm{br} \mathrm{s}, 2 \mathrm{H}), 3.80(\mathrm{~s}, 3 \mathrm{H}), 3.43(\operatorname{app~t}$, $J=6.5 \mathrm{~Hz}, 2 \mathrm{H}), 2.44(\mathrm{~m}, 1 \mathrm{H}), 1.59(\mathrm{~m}, 2 \mathrm{H}), 1.32(\mathrm{~m}, 6 \mathrm{H}), 0.99(\mathrm{~d}, J=6.7 \mathrm{~Hz}, 3 \mathrm{H}) ;{ }^{13} \mathrm{C} \mathrm{NMR}(125 \mathrm{MHz}$, $\left.\mathrm{CDCl}_{3}\right) \delta 159.1,144.3,130.7,129.1,113.7,87.2,72.5,70.0,55.2,38.2,36.0,29.6,26.9,26.2,19.1$; high resolution mass spectrum $\left(\mathrm{Cl}, \mathrm{NH}_{3}\right) \mathrm{m} / \mathrm{z} 418.0127\left[\mathrm{M}^{+}\right.$; calcd for $\mathrm{C}_{17} \mathrm{H}_{24} \mathrm{Br}_{2} \mathrm{O}_{2}^{+}:$: 418.0142].

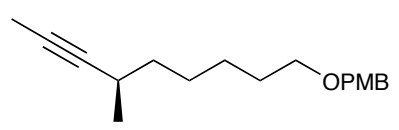

(-)-SI17

Alkyne (-)-Sl17. To a $-78{ }^{\circ} \mathrm{C}$ solution of azeotroped gem-dibromide (-)-Sl16 (6.65 g, 15.8 mmole) in THF (83 mL) was added $n$-BuLi $(27.0 \mathrm{~mL}, 2.5$ equiv., $1.47 \mathrm{M}$ in hexanes) dropwise over $\sim 5$ min. After $1 \mathrm{~h}$, the reaction was charged with Mel (4.9 mL, 5.0 equiv.), and after an additional $1 \mathrm{~h}$, was warmed to ambient temperature. Following an additional $2 \mathrm{~h}$, the reaction was diluted with water (125 $\mathrm{mL})$. The aqueous phase was then washed with $\mathrm{Et}_{2} \mathrm{O}(2 \times 50 \mathrm{~mL})$, and the combined organic layers were dried over $\mathrm{MgSO}_{4}$ and concentrated in vacuo. Purification by flash chromatography (20/1, hexanes/EtOAc) afforded $4.22 \mathrm{~g}(97 \%$ yield $)$ of $(-)$-SI17 as a pale yellow oil: $R_{f} 0.12(20 / 1$ hexanes/EtOAc); $[\alpha]_{D}^{20}-21.9\left(c 0.53, \mathrm{C}_{6} \mathrm{D}_{6}\right)$; IR (neat) $2932(\mathrm{~s}), 2856(\mathrm{~s}), 1612(\mathrm{~m}), 1512(\mathrm{~s}), 1456(\mathrm{w})$,

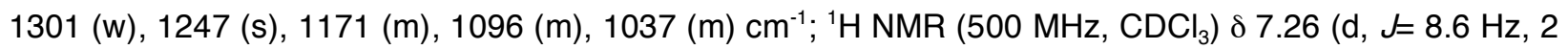
H), $6.87(\mathrm{~d}, J=8.6 \mathrm{~Hz}, 2 \mathrm{H}), 4.43(\mathrm{br} \mathrm{s}, 2 \mathrm{H}), 3.80(\mathrm{~s}, 3 \mathrm{H}), 3.44(\operatorname{app~t}, J=6.6 \mathrm{~Hz}, 2 \mathrm{H}), 2.34(\mathrm{~m}, 1 \mathrm{H})$, $1.78(\mathrm{~d}, \mathrm{~J}=2.3 \mathrm{~Hz}, 3 \mathrm{H}), 1.61(\mathrm{~m}, 2 \mathrm{H}), 1.30-1.50(\mathrm{~m}, 6 \mathrm{H}), 1.11(\mathrm{~d}, \mathrm{~J}=6.8 \mathrm{~Hz}, 3 \mathrm{H}) ;{ }^{13} \mathrm{C} \mathrm{NMR}(125 \mathrm{MHz}$, $\left.\mathrm{CDCl}_{3}\right) \delta 159.0,130.8,129.1,113.7,83.9,75.4,72.4,70.1,55.2,37.2,29.7,27.2,26.0,25.8,21.3,3.4$ high resolution mass spectrum (ES+) $\mathrm{m} / \mathrm{z} 297.1817$ [(M+Na) ${ }^{+}$; calcd for $\mathrm{C}_{18} \mathrm{H}_{26} \mathrm{O}_{2} \mathrm{Na}^{+}$: 297.1829]. 


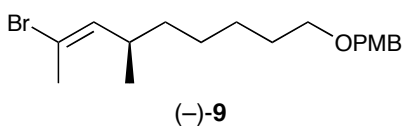

Vinyl Bromide (-)-9. To a $50{ }^{\circ} \mathrm{C}$ suspension of $\mathrm{Cp}_{2} \mathrm{ZrHCl}(1.77 \mathrm{~g}, 3.0$ equiv.) in toluene $(15 \mathrm{~mL})$ was added a solution of azeotroped alkyne (-)-SI17 (599 mg, $1.18 \mathrm{mmole})$ in toluene $(8 \mathrm{~mL})$. Gas Evolution! After $1 \mathrm{~h}$, the orange suspension was charged with $\mathrm{N}$-bromosuccinimide (1.32 g, 3.4 equiv.) in one portion and cooled to ambient temperature. After $45 \mathrm{~min}$, the brownish suspension was quenched with sat. $\mathrm{NaHCO}_{3}(30 \mathrm{~mL})$ and diluted with water $(30 \mathrm{~mL})$ and hexanes/EtOAc $(30 \mathrm{~mL}, 7 / 1)$. The aqueous phase was then washed with hexanes/EtOAc $(4 \times 25 \mathrm{~mL}, 7 / 1)$, and the combined organic layers were washed with brine $(25 \mathrm{~mL})$, dried over $\mathrm{MgSO}_{4}$, filtered through a pad of celite and concentrated in vacuo. Purification by flash chromatography $(30 / 1 \rightarrow 10 / 1$, hexanes/EtOAc) afforded $706 \mathrm{mg}(91 \%$ yield) of $(-)-9$ as a pale yellow oil: $\mathrm{R}_{\mathrm{f}} 0.19$ (20/1 hexanes/EtOAc); $[\alpha]_{\mathrm{D}}^{20}-29.6\left(c \quad 0.55, \mathrm{C}_{6} \mathrm{H}_{6}\right)$; IR (neat) $2928(\mathrm{~s}), 2854$ (s), $1612(\mathrm{~m}), 1512(\mathrm{~s}), 1463(\mathrm{~m}), 1302(\mathrm{~m}), 1247(\mathrm{~s}), 1172(\mathrm{~m}), 1098(\mathrm{~m}), 1037(\mathrm{~m}) \mathrm{cm}^{-1} ;{ }^{1} \mathrm{H}$ NMR $(500$ $\left.\mathrm{MHz}, \mathrm{CDCl}_{3}\right) \delta 7.25(\mathrm{~d}, J=8.5 \mathrm{~Hz}, 2 \mathrm{H}), 6.87(\mathrm{~d}, J=8.6 \mathrm{~Hz}, 2 \mathrm{H}), 5.61(\mathrm{dd}, J=10.0,1.2 \mathrm{~Hz}, 1 \mathrm{H}), 4.43(\mathrm{br}$ s, 2 H), 3.80 (s, 3 H), $3.42(\operatorname{app~t,~J=6.5~Hz,~} 2$ H), $2.30(m, 1$ H), $2.20(\mathrm{~s}, 3 \mathrm{H}), 1.59(\mathrm{~m}, 2 \mathrm{H}), 1.10-1.40$ (m, $6 \mathrm{H}), 0.96(\mathrm{~d}, \mathrm{~J}=6.7 \mathrm{~Hz}, 3 \mathrm{H}) ;{ }^{13} \mathrm{C}$ NMR $\left(125 \mathrm{MHz}, \mathrm{CDCl}_{3}\right) \delta 159.1,138.4,130.8,129.1,118.0,113.7$, $72.4,70.0,55.2,37.0,34.7,29.6,27.1,26.2,23.4,20.5$; high resolution mass spectrum $\left(\mathrm{Cl}, \mathrm{NH}_{3}\right) \mathrm{m} / \mathrm{z}$ $355.1149\left[\mathrm{M}^{+}\right.$; calcd for $\mathrm{C}_{18} \mathrm{H}_{27} \mathrm{BrO}_{2}^{+}:$355.1193].

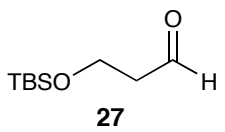

Aldehyde 27. During all additions, the internal reaction temperature was kept below $-60{ }^{\circ} \mathrm{C}$. To a $-78{ }^{\circ} \mathrm{C}$ solution of oxalyl chloride $\left(72.7 \mathrm{~mL}, 2 \mathrm{M}\right.$ in $\mathrm{CH}_{2} \mathrm{Cl}_{2}, 1.5$ equiv.) in $\mathrm{CH}_{2} \mathrm{Cl}_{2}(500 \mathrm{~mL})$ was added DMSO (22.0 mL, 3.2 equiv.) dropwise via addition funnel. Gas Evolution! After $30 \mathrm{~min}$, a solution of azeotroped alcohol SI6 (18.5 g, 96.9 mmole) in $\mathrm{CH}_{2} \mathrm{Cl}_{2}(500 \mathrm{~mL})$ was added via cannula. Following an additional $30 \mathrm{~min}$, the cloudy suspension was treated with triethylamine $(67.5 \mathrm{~mL}, 5.0$ equiv. $)$ to produce a homogeneous solution. Upon warming to $0{ }^{\circ} \mathrm{C}$, a yellow suspension reformed and after $2.5 \mathrm{~h}$ was quenched with $50 \%$ sat, $\mathrm{NaHCO}_{3}(250 \mathrm{~mL})$. The organic phase was then washed with additional $50 \%$ sat. $\mathrm{NaHCO}_{3}(250 \mathrm{~mL})$ and concentrated in vacuo to $\sim 1 / 2$ volume. The remaining aqueous layers were 
then washed with diethyl ether $(3 \times 150 \mathrm{~mL})$, and the combined organic layers were washed with $1 \mathrm{M}$ $\mathrm{NaHSO}_{4}(2 \times 150 \mathrm{~mL}), 50 \%$ sat. $\mathrm{NaHCO}_{3}(150 \mathrm{~mL})$, brine $(150 \mathrm{~mL})$, dried over $\mathrm{MgSO}_{4}$ and concentrated in vacuo to afford an orange oil. Purification by flash chromatography (10/1 hexanes/EtOAc) afforded $16.8 \mathrm{~g}$ (92\% yield) of 27 as a colorless oil: $\mathrm{R}_{\mathrm{f}} 0.22$ (10/1 hexanes/EtOAc); IR (thin film, $\left.\mathrm{CDCl}_{3}\right) 2956$ (s), 2929 (s), 2857 (s), 1728 (s), 1472 (m), 1256 (s), 1098 (s) cm ${ }^{-1} ;{ }^{1} \mathrm{H}$ NMR $\left(500 \mathrm{MHz}, \mathrm{CDCl}_{3}\right) \delta 9.78$ (t, J= $2.1 \mathrm{~Hz}, 1 \mathrm{H}), 3.97(\mathrm{t}, J=6.0 \mathrm{~Hz}, 2 \mathrm{H}), 2.58(\mathrm{dt}, J=6.0,2.1 \mathrm{~Hz}, 2 \mathrm{H}), 0.86(\mathrm{~s}, 9 \mathrm{H}), 0.05(\mathrm{~s}, 6 \mathrm{H}) ;{ }^{13} \mathrm{C} \mathrm{NMR}$ $\left(125 \mathrm{MHz}, \mathrm{CDCl}_{3}\right) \delta 201.8,57.3,46.5,25.7,18.1,-5.4$; high resolution mass spectrum $\left(\mathrm{Cl}, \mathrm{NH}_{3}\right) \mathrm{m} / \mathrm{z}$ 189.1321 [MH+'; calcd for $\mathrm{C}_{9} \mathrm{H}_{21} \mathrm{O}_{2} \mathrm{Si}^{+}:$189.1232].

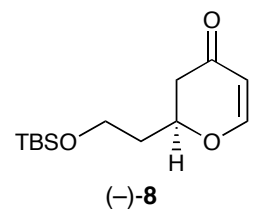

Enone (-)-8. To a mixture of aldehyde 27 (11.3 g, 59.9 mmoles), $\operatorname{Cr}($ III) catalyst 28 (433 mg, 0.015 equiv.) and powdered $4 \AA$ molecular sieves $\left(11.9 \mathrm{~g}\right.$, oven-dried at $>100{ }^{\circ} \mathrm{C}$ for $3 \mathrm{~h}$ and cooled under high vacuum) was added distilled acetone $(11.9 \mathrm{~mL})$. The reaction flask was then wrapped in Al foil, and the brown suspension was treated with Danishefsky's diene (17.5 mL, 1.5 equiv.) dropwise. After $19 \mathrm{~h}$, the reaction was diluted with $\mathrm{CH}_{2} \mathrm{Cl}_{2}(65 \mathrm{~mL})$, cooled to $0{ }^{\circ} \mathrm{C}$ and charged with trifluoroacetic acid $(6.9 \mathrm{~mL}$, 1.5 equiv.) dropwise. After $1 \mathrm{~h}$, the suspension was filtered through celite, and the eluent washed with sat. $\mathrm{NaHCO}_{3}(100 \mathrm{~mL})$. The aqueous phase was then washed with $\mathrm{CH}_{2} \mathrm{Cl}_{2}(3 \times 30 \mathrm{~mL})$, and the combined organic layers were washed with brine $(30 \mathrm{~mL})$, dried over $\mathrm{MgSO}_{4}$ and concentrated in vacuo to afford a dark orange oil. Purification by flash chromatography (6/1, hexanes/EtOAc) afforded $15.2 \mathrm{~g}(98 \%$ yield) of (-)-8 as an orange oil: $\mathrm{R}_{\mathrm{f}} 0.21\left(6 / 1\right.$ hexanes/EtOAc); $[\alpha]_{\mathrm{D}}^{20}-70.0\left(c 1.00, \mathrm{CH}_{2} \mathrm{Cl}_{2}\right)$; IR (neat) 2955 (s), 2928 (s), 2884 (s), 2857 (s), 1686 (s), 1682 (s), 1598 (s), 1471 (m), 1463 (m), 1404 (s), 1361 (w), 1274 (s), 1213 (s), 1094 (s) cm ${ }^{-1} ;{ }^{1} \mathrm{H}$ NMR $\left(500 \mathrm{MHz}, \mathrm{CDCl}_{3}\right) \delta 7.34$ (d, J=6.0 Hz, $1 \mathrm{H}$ ), 5.40 (dd, J=6.0, $1.0 \mathrm{~Hz}, 1 \mathrm{H}), 4.61(\mathrm{~m}, 1 \mathrm{H}), 3.79(\mathrm{~m}, 2 \mathrm{H}), 2.45-2.58(\mathrm{~m}, 2 \mathrm{H}), 2.04(\mathrm{~m}, 1 \mathrm{H}), 1.86(\mathrm{~m}, 1 \mathrm{H}), 0.89(\mathrm{~s}, 9 \mathrm{H})$, 0.05 (s, $6 \mathrm{H}) ;{ }^{13} \mathrm{C}$ NMR $\left(125 \mathrm{MHz}, \mathrm{CDCl}_{3}\right) \delta 192.5,163.0,107.1,76.5,58.3,42.0,37.4,25.9,18.2,-5.3$, 5.4; high resolution mass spectrum $\left(\mathrm{Cl}, \mathrm{NH}_{3}\right) \mathrm{m} / \mathrm{z} 257.1585\left[\mathrm{MH}^{+}\right.$; calcd for $\mathrm{C}_{13} \mathrm{H}_{25} \mathrm{O}_{3} \mathrm{Si}^{+}$: 257.1572]. 


\section{Determination of Enantiomeric Purity and Stereochemistry Verification of (-)-8.}

HPLC conditions: CHIRACEL-OD, $0.75 \mathrm{~mL} / \mathrm{min} ., 2 \%$ IPA in hexanes, $25^{\circ} \mathrm{C}, \lambda=250 \mathrm{~nm}$.

\begin{tabular}{|c|c|c|c|c|}
\hline Compound & Retention Time $\left(\mathrm{R}_{\mathrm{t}}\right)$ & \% Area & Retention Time $\left(\mathrm{R}_{\mathrm{t}}\right)$ & $\%$ Area \\
\hline racemic & 11.37 min. & 49.9 & $12.48 \mathrm{~min}$. & 50.1 \\
\hline$(-)-8$ & $11.30 \mathrm{~min}$. & 5.0 & $12.16 \mathrm{~min}$. & 95.0 \\
\hline
\end{tabular}

The retention times for $(-)-\mathbf{8}$ were identical to those previously reported for this compound ${ }^{5}$ and thus verified the correct absolute stereochemistry.

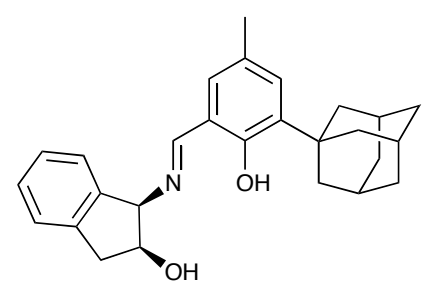

(+)-SI18

Imine (+)-SI18. To a solution of 2-adamantyl-1-p-cresol (1.56 g, 6.44 mmoles) in toluene (14 mL) was sequentially added distilled 2,6-lutidine (600 $\mu \mathrm{L}, 0.8$ equiv.) and tin(IV) chloride (151 $\mu \mathrm{L}, 0.2$ equiv.). After $20 \mathrm{~min}$, the reaction was charged with paraformaldehyde $(0.77 \mathrm{~g}, 4.0$ equiv. $)$ and then heated to reflux. After $6 \mathrm{~h}$, the yellow suspension was cooled to ambient temperature and filtered through a plug of Si gel/celite (1/1, $1.5 \mathrm{~g}$ total). The plug was then washed with EtOAc $(25 \mathrm{~mL})$, and the eluent was washed with water $(45 \mathrm{~mL}), 1 \mathrm{~N} \mathrm{HCl}(45 \mathrm{~mL})$, brine $(45 \mathrm{~mL})$, dried over $\mathrm{Na}_{2} \mathrm{SO}_{4}$ and concentrated in vacuo to afford $1.71 \mathrm{~g}$ (98\% yield) of a yellow solid corresponding to the aldehyde which was used without further purification. The aldehyde was then charged with absolute EtOH $(25 \mathrm{~mL})$ and heated to reflux. (1R, 2S)2-aminoindanol ( $0.99 \mathrm{~g}, 1.05$ equiv.) was then added in one portion and after $45 \mathrm{~min}$, the suspension was cooled to ambient temperature. After an additional $3 \mathrm{~h}$, the reaction was filtered and the solid was washed with cold EtOH $(2 \times 5 \mathrm{~mL})$. The remaining bright yellow solid was then dried under high vacuum to afford $2.07 \mathrm{~g}\left(80 \%\right.$ yield, 2 steps) of $(+)$-SI18: $\mathrm{mp} 219-221^{\circ} \mathrm{C} ;[\alpha]_{\mathrm{D}}^{20}+64.0(c 0.50$, THF); IR (thin film, $\mathrm{CH}_{2} \mathrm{Cl}_{2}$ ) 3389 (br, w), 2902 (s), 2847 (m), 1625 (m), $1594(\mathrm{~m}), 1453(\mathrm{~m}) \mathrm{cm}^{-1} ;{ }^{1} \mathrm{H}$ NMR (500 MHz, d ${ }^{-}$ DMSO) $\delta 14.16(\mathrm{~s}, 1 \mathrm{H}), 8.59(\mathrm{~s}, 1 \mathrm{H}), 7.29(\mathrm{~m}, 1 \mathrm{H}), 7.24(\mathrm{~m}, 1 \mathrm{H}), 7.18(\mathrm{~m}, 2 \mathrm{H}), 7.08(\mathrm{~s}, 1 \mathrm{H}), 7.00(\mathrm{~d}$, 
$J=1.4 \mathrm{~Hz}, 1 \mathrm{H}), 5.20(\mathrm{~d}, J=5.0 \mathrm{~Hz}, 1 \mathrm{H}), 4.72(\mathrm{~d}, J=5.5 \mathrm{~Hz}, 1 \mathrm{H}), 4.53(\mathrm{~m}, 1 \mathrm{H}), 3.09(\mathrm{dd}, J=15.5,6.1 \mathrm{~Hz}$, $1 \mathrm{H}), 2.92(\mathrm{dd}, J=15.5,6.0 \mathrm{~Hz}, 1 \mathrm{H}), 2.22(\mathrm{~s}, 3 \mathrm{H}), 2.04(\mathrm{~m}, 6 \mathrm{H}), 1.98(\mathrm{~s}, 3 \mathrm{H}), 1.68(\mathrm{~m}, 6 \mathrm{H}) ;{ }^{13} \mathrm{C}$ NMR (125 MHz, $\mathrm{d}_{6}$-DMSO) $\delta 166.5,158.5,142.0,141.0,136.4,130.0,129.6,128.0,126.6,125.7,125.1$, $124.7,118.2,73.9,39.7,39.0,36.5,36.2,28.3,20.3$; high resolution mass spectrum (ES+) m/z 402.2636 $\left[\mathrm{MH}^{+}\right.$; calcd for $\mathrm{C}_{27} \mathrm{H}_{32} \mathrm{NO}_{2}$ : 402.2433].

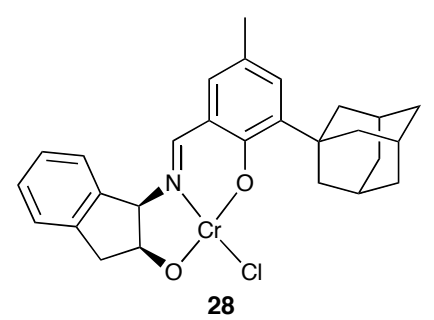

Chromium (III) Complex 28. To a solution of imine (+)-Sl18 (2.05 g, 5.11 mmoles) and chromium(III) chloride tetrahydrofuran complex (1:3) (1.97 g, 1.0 equiv.) in $\mathrm{CH}_{2} \mathrm{Cl}_{2}$ (41 mL) was added distilled 2,6-lutidine (1.2 mL, 2.0 equiv.). Gas Evolution! After $3 \mathrm{~h}$, the dark brown solution was diluted with $\mathrm{CH}_{2} \mathrm{Cl}_{2}(200 \mathrm{~mL})$, and the organic phase was washed with water $(3 \times 120 \mathrm{~mL})$, brine $(120 \mathrm{~mL})$, dried over $\mathrm{Na}_{2} \mathrm{SO}_{4}$ and concentrated in vacuo. The brown residue was then washed with cold acetone ( 3 x 5 $\mathrm{mL}$ ), filtered and air-dried to afford $1.63 \mathrm{~g}$ (65\% yield) of $\mathbf{2 8}$ as a brown powder that was used without further purification.

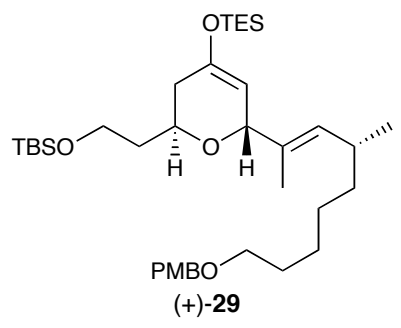

TES-enol ether (+)-29. To a $-78^{\circ} \mathrm{C}$ solution of $t$-BuLi (1.24 mL, $1.49 \mathrm{M}$ in pentane, 5.28 equiv.) in THF (7.4 mL) was added a solution of azeotroped vinyl bromide (-)-9 (300 mg, 2.4 equiv.) in THF (1.6 $\mathrm{mL}$ ) dropwise. After $1 \mathrm{~h}$, the yellow solution was added via cannula to a non-stirring, $-78^{\circ} \mathrm{C}$ suspension of CuCN (52.9 mg, 1.68 equiv.) in THF (6.6 mL), followed by a $1.0 \mathrm{~mL}$ THF rinse. Stirring was then initiated, and the suspension was placed in a $0{ }^{\circ} \mathrm{C}$ bath for $5 \mathrm{~min}$. The resulting green/brown solution was then recooled to $-78{ }^{\circ} \mathrm{C}$, and sequentially treated with a solution of azeotroped enone (-)-8 (89.4 $\mathrm{mg}, 0.352$ 
mmoles) in THF (485 $\mu \mathrm{L})$, followed by freshly distilled triethylsilyl chloride (118 $\mu \mathrm{L}, 2.0$ equiv.). After 40 min., the reaction was quenched with $\mathrm{CD}_{3} \mathrm{OD}(500 \mu \mathrm{L})$, and warmed to ambient temperature. Sat. $\mathrm{NaHCO}_{3}(25 \mathrm{~mL})$, and $\mathrm{CH}_{2} \mathrm{Cl}_{2}(25 \mathrm{~mL})$ were then added, and the aqueous phase was washed with $\mathrm{CH}_{2} \mathrm{Cl}_{2}$ $(3 \times 5 \mathrm{~mL})$. The combined organic layers were then dried over $\mathrm{Na}_{2} \mathrm{SO}_{4}$ and concentrated in vacuo. Purification by flash chromatography (15/1, hexanes/diethyl ether, prepared column with $3 \% \mathrm{Et}_{3} \mathrm{~N}$ in $15 / 1$, hexanes/diethyl ether) afforded $140 \mathrm{mg}(61 \%$ yield $)$ of $(+)-29: \mathrm{R}_{\mathrm{f}} 0.40\left(10 / 1\right.$ hexanes/EtOAc); $[\alpha]_{\mathrm{D}}^{20}+6.2(c$ 0.45, $\mathrm{C}_{6} \mathrm{H}_{6}$ ); IR (thin film, $\mathrm{CDCl}_{3}$ ) 2953 (s), 2927 (s), 2875 (s), 2855 (s), 1674 (m), 1603 (m), 1513 (m),

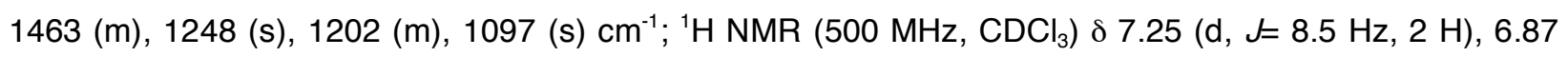
(d, J= 8.5 Hz, $2 \mathrm{H}), 5.12(\mathrm{~d}, J=9.4 \mathrm{~Hz}, 1 \mathrm{H}), 4.83(\mathrm{~d}, J=3.4 \mathrm{~Hz}, 1 \mathrm{H}), 4.51$ (br s, $1 \mathrm{H}), 4.42(\mathrm{br} \mathrm{s}, 2 \mathrm{H})$, $3.80(\mathrm{~s}, 3 \mathrm{H}), 3.73(\mathrm{~m}, 2 \mathrm{H}), 3.65(\mathrm{~m}, 1 \mathrm{H}), 3.42(\operatorname{app~t}, J=6.6 \mathrm{~Hz}, 2 \mathrm{H}), 2.34(\mathrm{~m}, 1 \mathrm{H}), 2.03$ (dd, J= 16.6, 8.4 Hz, $1 \mathrm{H}), 1.95(\mathrm{dd}, J=16.6,3.9 \mathrm{~Hz}, 1 \mathrm{H}), 1.79(\mathrm{~m}, 1 \mathrm{H}), 1.66(\mathrm{~m}, 1 \mathrm{H}), 1.65(\mathrm{~s}, 3 \mathrm{H}), 1.57(\mathrm{~m}, 2 \mathrm{H})$, 1.17-1.38 (m, $6 \mathrm{H}), 0.99$ (t, J= $7.9 \mathrm{~Hz}, 9 \mathrm{H}), 0.90$ (d, J= 6.6 Hz, $3 \mathrm{H}), 0.87$ (s, $9 \mathrm{H}), 0.69$ (q, J= $7.9 \mathrm{~Hz}, 6$ $\mathrm{H}), 0.03(\mathrm{~s}, 6 \mathrm{H}) ;{ }^{13} \mathrm{C}$ NMR $\left(125 \mathrm{MHz}, \mathrm{CDCl}_{3}\right) \delta 159.0,148.5,136.0,133.6,130.8,129.1,113.7,103.5$, $76.3,72.4,70.2,65.2,60.1,55.2,38.5,37.4,36.0,32.3,29.8,27.4,26.3,25.9,20.8,18.2,14.5,6.6,5.0$, 5.3; high resolution mass spectrum (ES+) $\mathrm{m} / \mathrm{z} 669.4321$ [( $\mathrm{M}+\mathrm{Na})^{+}$; calcd for $\mathrm{C}_{37} \mathrm{H}_{66} \mathrm{O}_{5} \mathrm{Si}_{2} \mathrm{Na}^{+}$: 669.4449].

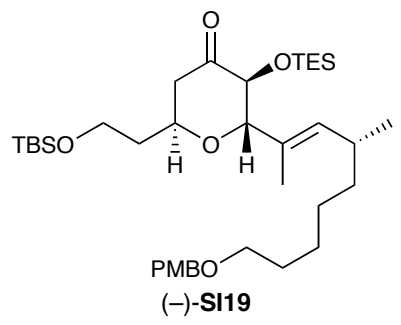

TES-ether (-)-S119. To a $0{ }^{\circ} \mathrm{C}$ suspension of TES-enol ether (+)-29 (272 mg, 420 mmoles), and $\mathrm{NaHCO}_{3}$ (98.8 mg, 2.8 equiv.) in $\mathrm{CH}_{2} \mathrm{Cl}_{2}(11.8 \mathrm{~mL}$ ) was added $m$-CPBA (94.1 mg, 77\% max., 1.0 equiv.) in one portion. After $20 \mathrm{~min}$, the reaction was allowed to warm to ambient temperature, and after an additional $1 \mathrm{~h}$ was quenched with $1 \mathrm{M} \mathrm{Na}_{2} \mathrm{~S}_{2} \mathrm{O}_{3}(20 \mathrm{~mL})$ and EtOAc $(20 \mathrm{~mL})$. The organic layer was then washed with sat. $\mathrm{NaHCO}_{3}(4 \times 20 \mathrm{~mL})$, brine $(10 \mathrm{~mL})$, dried over $\mathrm{MgSO}_{4}$ and concentrated in vacuo. Purification by flash chromatography $\left(20 / 1 \rightarrow 5 / 1\right.$, hexanes/diethyl ether, prepared column with $3 \% \mathrm{Et}_{3} \mathrm{~N}$ in 20/1, hexanes/diethyl ether) afforded $128.4 \mathrm{mg}$ (46\% yield) of (-)-SI19, and $39.6 \mathrm{mg}$ (15\% recovery) of 
(+)-29 as pale yellow oils. For (-)-SI19: $\mathrm{R}_{\mathrm{f}} 0.48$ (4/1 hexanes/EtOAc); $[\alpha]_{\mathrm{D}}^{20}-39.1\left(c 0.92, \mathrm{C}_{6} \mathrm{H}_{6}\right)$; IR (thin film, $\left.\mathrm{CDCl}_{3}\right) 2930$ (s), $2900(\mathrm{~m}), 1730(\mathrm{~m}), 1613(\mathrm{w}), 1513(\mathrm{w}), 1463(\mathrm{w}), 1248(\mathrm{~m}), 1147(\mathrm{~m}), 1097(\mathrm{~s}) \mathrm{cm}^{-}$ 1; ${ }^{1} \mathrm{H}$ NMR (500 MHz, $\left.\mathrm{C}_{6} \mathrm{D}_{6}\right) \delta 7.25(\mathrm{~d}, J=8.5 \mathrm{~Hz}, 2 \mathrm{H}), 6.87$ (d, J=8.5 Hz, $\left.2 \mathrm{H}\right), 5.22(\mathrm{~d}, J=8.8 \mathrm{~Hz}, 1 \mathrm{H})$, 4.42 (br s, 2 H), 4.36 (m, 1 H), 4.17 (d, J= $7.9 \mathrm{~Hz}, 1 \mathrm{H}), 3.91$ (d, J= $7.9 \mathrm{~Hz}, 1 \mathrm{H}), 3.80$ (s, $3 \mathrm{H}), 3.64$ (m, 2 H), $3.42(\operatorname{appt}, J=6.6 \mathrm{~Hz}, 2 \mathrm{H}), 2.71(\mathrm{dd}, J=13.6,6.4 \mathrm{~Hz}, 1 \mathrm{H}), 2.43(\mathrm{dd}, J=13.6,3.3 \mathrm{~Hz}, 1 \mathrm{H}), 2.36(\mathrm{~m}, 1$ H), $1.84(\mathrm{~m}, 1 \mathrm{H}), 1.69(\mathrm{~s}, 3 \mathrm{H}), 1.60(\mathrm{~m}, 3 \mathrm{H}), 1.28(\mathrm{br} \mathrm{m}, 6 \mathrm{H}), 0.92(\mathrm{~m}, 12 \mathrm{H}), 0.87(\mathrm{~s}, 9 \mathrm{H}), 0.60(\mathrm{~m}, 6$ H), 0.02 (s, $6 \mathrm{H}) ;{ }^{13} \mathrm{C}$ NMR $\left(125 \mathrm{MHz}, \mathrm{C}_{6} \mathrm{D}_{6}\right) \delta$ 205.5, 159.0, 137.7, 130.8, 129.9, 129.1, 113.7, 82.8, 76.3, $72.4,70.4,70.2,59.1,55.2,45.3,37.1,35.2,32.2,29.7,27.2,26.4,25.9,20.3,18.3,12.1,6.8,4.9,-5.4$; high resolution mass spectrum (ES+) $\mathrm{m} / \mathrm{z} 685.4293$ [(M+Na) ${ }^{+}$; calcd for $\mathrm{C}_{37} \mathrm{H}_{66} \mathrm{O}_{6} \mathrm{Si}_{2} \mathrm{Na}^{+}$: 685.4398].

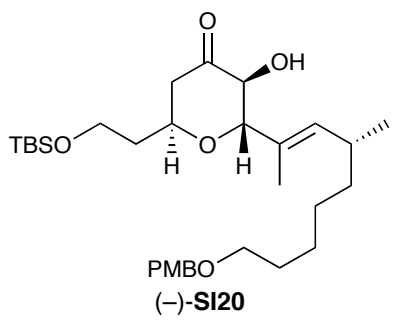

Alcohol (-)-SI20. To a $0{ }^{\circ} \mathrm{C}$ solution of TES-ether (-)-SI19 (162.5 mg, $\left.0.245 \mathrm{mmole}\right)$ in $\mathrm{MeOH}$ (6.5 mL) was added PPTS (12.3 mg, 0.2 equiv.). After $4 \mathrm{~h}$, the reaction was diluted with EtOAc $(15 \mathrm{~mL})$, sat. $\mathrm{NaHCO}_{3}(5 \mathrm{~mL})$ and water $(15 \mathrm{~mL})$. The aqueous layer was then washed with EtOAc $(3 \times 5 \mathrm{~mL})$ and the combined organic layers were washed with brine $(10 \mathrm{~mL})$, dried over $\mathrm{MgSO}_{4}$ and concentrated in vacuo under high vacuum overnight to afford $134.1 \mathrm{mg}$ (100\% yield) of (-)-SI20 as a colorless oil which was used without further purification: $\mathrm{R}_{\mathrm{f}} 0.27$ (4/1 hexanes/EtOAc); $[\alpha]_{D}^{20}-43.2\left(c 0.43, \mathrm{C}_{6} \mathrm{H}_{6}\right)$; IR (neat) 3476 (br, m), 2931 (s), 2910 (s), 1721 (s), 1612 (m), 1513 (m), 1463 (m), 1250 (s), 1102 (s) cm cm $^{-1}{ }^{1} \mathrm{H}$ NMR $\left(500 \mathrm{MHz}, \mathrm{CDCl}_{3}\right) \delta 7.25(\mathrm{~d}, J=8.6 \mathrm{~Hz}, 2 \mathrm{H}), 6.86(\mathrm{~d}, \mathrm{~J}=8.6 \mathrm{~Hz}, 2 \mathrm{H}), 5.25$ (dd, J= 9.5, $\left.1.0 \mathrm{~Hz}, 1 \mathrm{H}\right), 4.55$ (m, $1 \mathrm{H}), 4.41$ (br s, $2 \mathrm{H}), 4.07$ (dd, J= 9.4, $2.7 \mathrm{~Hz}, 1 \mathrm{H}), 3.79(\mathrm{~s}, 3 \mathrm{H}), 3.72(\mathrm{~d}, \mathrm{~J}=9.4 \mathrm{~Hz}, 1 \mathrm{H}), 3.64(\mathrm{~m}, 2$ H), 3.42 (m, 3 H), 2.91 (ddd, J= 13.7, 7.4, 1.3 Hz, 1 H), 2.49 (dd, J=13.7, $1.5 \mathrm{~Hz}, 1 \mathrm{H}), 2.41(\mathrm{~m}, 1 \mathrm{H}), 1.81$ (dddd, J= 10.8, 9.1, 5.4, $5.4 \mathrm{~Hz}, 1 \mathrm{H}), 1.73(\mathrm{~d}, J=1.0 \mathrm{~Hz}, 3 \mathrm{H}), 1.59(\mathrm{~m}, 4 \mathrm{H}), 1.28(\mathrm{~m}, 5 \mathrm{H}), 0.95(\mathrm{~d}, \mathrm{~J}=$ $6.6 \mathrm{~Hz}, 3 \mathrm{H}), 0.88(\mathrm{~s}, 9 \mathrm{H}), 0.03(\mathrm{~s}, 6 \mathrm{H}) ;{ }^{13} \mathrm{C} \operatorname{NMR}\left(125 \mathrm{MHz}, \mathrm{CDCl}_{3}\right) \delta 206.0,159.1,137.8,130.8,129.7$, 129.2, 113.7, 82.6, 74.8, 72.4, 71.3, 70.1, 59.1, 55.2, 44.3, 37.3, 34.4, 32.1, 29.6, 27.0, 26.2, 25.9, 20.6, 
18.3, 11.5, -5.4; high resolution mass spectrum (ES+) $\mathrm{m} / \mathrm{z} 571.3452\left[(\mathrm{M}+\mathrm{Na})^{+}\right.$; calcd for $\mathrm{C}_{31} \mathrm{H}_{52} \mathrm{O}_{6} \mathrm{SiNa}^{+}$: 571.3533].

Determination of Absolute Stereochemistry of (-)-SI20. (R)-(-)- $\alpha$-methoxy- $\alpha$-trifluoromethylphenyl-acetyl chloride [(R)-MTPACl, $6.6 \mu \mathrm{L}, 2.0$ equiv.) was added to a solution of (-)-SI20 (9.7 mg, 17.6 umoles) and 4-( $\mathrm{N}, \mathrm{N}$-dimethylamino)pyridine (8.6 mg, 4.0 equiv.) in $\mathrm{CH}_{2} \mathrm{Cl}_{2}(250 \mu \mathrm{L}$ ) at ambient temperature. After $3 \mathrm{~h}$, direct purification by Preparative-TLC (4/1, hexanes/EtOAc, $500 \mu \mathrm{m}$ plate) afforded $10.5 \mathrm{mg}$ (78\% yield, $>20: 1$ ratio of diastereomers) of the (S)-MTPA ester of (-)-SI20 as a colorless oil: $\mathrm{R}_{\mathrm{f}} 0.38\left(4 / 1\right.$, hexanes/EtOAc ); $[\alpha]_{\mathrm{D}}^{20}-23.8\left(c 0.50, \mathrm{C}_{6} \mathrm{H}_{6}\right)$; IR (thin film, $\left.\mathrm{CDCl}_{3}\right) 2930$ (s), 2855 (s), 1760 (s), 1734 (s), 1615 (w), 1513 (s), 1463 (m), 1361 (w), 1249 (s), 1171 (s), 1099 (s), 1035 (w) $\mathrm{cm}^{-1}$; ${ }^{1} \mathrm{H}$ NMR $\left(500 \mathrm{MHz}, \mathrm{CDCl}_{3}\right) \delta 7.67(\mathrm{~m}, 2 \mathrm{H}), 7.39(\mathrm{~m}, 3 \mathrm{H}), 7.26(\mathrm{~d}, J=8.5 \mathrm{~Hz}, 2 \mathrm{H}), 6.87(\mathrm{~d}, J=$ $8.5 \mathrm{~Hz}, 2 \mathrm{H}), 5.34(\mathrm{~d}, \mathrm{~J}=9.7 \mathrm{~Hz}, 1 \mathrm{H}), 5.24(\mathrm{~d}, J=9.1 \mathrm{~Hz}, 1 \mathrm{H}), 4.53(\mathrm{~m}, 1 \mathrm{H}), 4.43(\mathrm{br} \mathrm{s}, 2 \mathrm{H}), 4.12(\mathrm{~d}, J=$ $9.7 \mathrm{~Hz}, 1 \mathrm{H}$ ), $3.80(\mathrm{~s}, 3 \mathrm{H}), 3.64(\mathrm{~m}, 2 \mathrm{H}), 3.59(\mathrm{~s}, 3 \mathrm{H}), 3.42(\operatorname{app~t}, J=6.6 \mathrm{~Hz}, 2 \mathrm{H}), 2.99$ (dd, J = 13.9, 7.2 Hz, $1 \mathrm{H}), 2.51$ (dd, J=13.9, $1.7 \mathrm{~Hz}, 1 \mathrm{H}), 2.24(\mathrm{~m}, 1 \mathrm{H}), 1.87$ (dddd, J= 5.2, 5.2, 10.1, $10.1 \mathrm{~Hz}, 1 \mathrm{H}$ ), $1.66(\mathrm{~m}, 1 \mathrm{H}), 1.58(\mathrm{~s}, 3 \mathrm{H}), 1.56(\mathrm{~m}, 2 \mathrm{H}), 1.27(\mathrm{~m}, 2 \mathrm{H}), 1.16(\mathrm{~m}, 2 \mathrm{H}), 1.10(\mathrm{~m}, 2 \mathrm{H}), 0.87(\mathrm{~m}, 12 \mathrm{H})$, 0.03 (s, $6 \mathrm{H}) ;{ }^{13} \mathrm{C}$ NMR $\left(125 \mathrm{MHz}, \mathrm{CDCl}_{3}\right) \delta 200.2,165.6,159.0,139.1,131.8,131.8,129.6,129.1,128.6$, $128.2,127.7,124.2,121.9,113.7,78.8,77.1,72.4,71.0,70.1,58.9,55.5,55.2,45.4,36.5,34.4,32.0$, 29.6, 26.8, 26.3, 25.9, 19.8, 18.3, 11.7, -5.4; high resolution mass spectrum (ES+) m/z 787.3862 $\left[(\mathrm{M}+\mathrm{Na})^{+}\right.$; calcd for $\left.\mathrm{C}_{41} \mathrm{H}_{59} \mathrm{~F}_{3} \mathrm{O}_{8} \mathrm{SiNa}: 787.3931\right]$.

In similar fashion, the $(R)$-MTPA ester of $(-)$-SI20 was obtained in $57 \%$ yield $(>20: 1$ mixture of diastereomers) as a colorless oil: $\mathrm{R}_{\mathrm{f}} 0.38\left(4 / 1\right.$, hexanes/EtOAc ); $[\alpha]_{D}^{20}-12.5\left(c 0.35, \mathrm{C}_{6} \mathrm{H}_{6}\right)$; IR (thin film, $\left.\mathrm{CDCl}_{3}\right) 2930$ (s), 2856 (s), 1759 (s), 1734 (s), 1612 (w), $1513(\mathrm{~m}), 1464$ (m), 1361 (m), 1249 (s), 1171 (s),

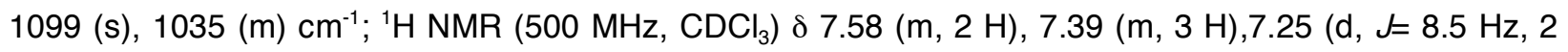
H), 6.87 (d, J= 8.5 Hz, 2 H), $5.33(\mathrm{~d}, J=9.6 \mathrm{~Hz}, 2 \mathrm{H}), 4.54(\mathrm{~m}, 1 \mathrm{H}), 4.41$ (br s, $2 \mathrm{H}), 4.19$ (d, J= 9.6 Hz, 1 H), $3.79(\mathrm{~s}, 3 \mathrm{H}), 3.64(\mathrm{~m}, 2 \mathrm{H}), 3.54(\mathrm{~s}, 3 \mathrm{H}), 3.40$ (app t, J = 6.6 Hz, 2 H), 2.97 (dd, J= 13.9, $7.1 \mathrm{~Hz}, 1 \mathrm{H}$ ), 2.49 (dd, J= 13.9, $2.0 \mathrm{~Hz}, 1 \mathrm{H}), 2.37$ (m, $1 \mathrm{H}), 1.86$ (dddd, J= 5.1, 5.1, 9.6, 9.6 Hz, $1 \mathrm{H}), 1.73$ (s, $3 \mathrm{H}), 1.65$ $(\mathrm{m}, 1 \mathrm{H}), 1.53(\mathrm{~m}, 2 \mathrm{H}), 1.25(\mathrm{~m}, 6 \mathrm{H}), 0.93(\mathrm{~d}, \mathrm{~J}=6.6 \mathrm{~Hz}, 3 \mathrm{H}), 0.87(\mathrm{~s}, 9 \mathrm{H}), 0.03(\mathrm{~s}, 6 \mathrm{H}) ;{ }^{13} \mathrm{C}$ NMR $(125$ 
$\mathrm{MHz}, \mathrm{CDCl}_{3}$, one carbon overlapping with $\left.\mathrm{CDCl}_{3}\right) \delta 199.6,165.5,159.1,139.3,131.8,130.8,129.6$, $129.1,128.7,128.2,127.8,124.2,121.9,113.7,79.0,72.4,71.0,70.1,58.9,55.5,55.2,45.3,36.8,34.5$, $32.3,29.6,27.0,26.3,25.9,20.1,18.3,11.7,-5.4$; high resolution mass spectrum (ES+) $\mathrm{m} / \mathrm{z} 787.3843$ $\left[(\mathrm{M}+\mathrm{Na})^{+}\right.$; calcd for $\left.\mathrm{C}_{41} \mathrm{H}_{59} \mathrm{~F}_{3} \mathrm{O}_{8} \mathrm{SiNa}: 787.3931\right]$.

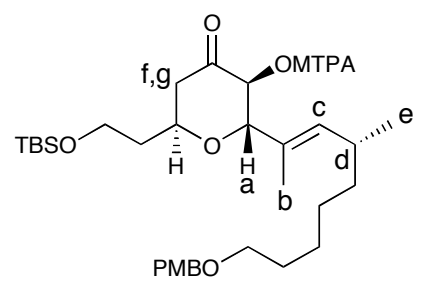

\begin{tabular}{|c|c|c|c|}
\hline Proton & $(S)$-MTPA ester & $(R)$-MTPA ester & $(S)-(R)$ \\
\hline a & 4.12 & 4.19 & -0.07 \\
\hline b & 1.58 & 1.73 & -0.15 \\
\hline c & 5.24 & 5.33 & -0.09 \\
\hline d & 2.24 & 2.37 & -0.13 \\
\hline e & 0.87 & 0.93 & -0.06 \\
\hline f & 2.99 & 2.97 & +0.02 \\
\hline g & 2.51 & 2.49 & +0.02 \\
\hline
\end{tabular}

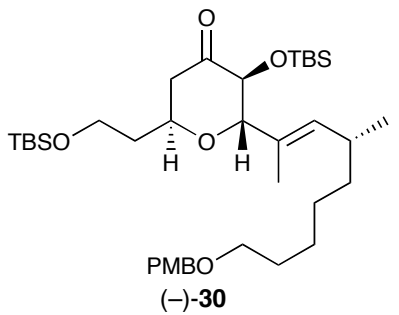

Bis-TBS ether $(-) 30$. To a $-78{ }^{\circ} \mathrm{C}$ solution of $(-)$-SI20 (133.4 mg, $\left.0.243 \mathrm{mmole}\right)$ and distilled 2,4,6-collidine ( $97 \mu \mathrm{L}, 3.0$ equiv.) was added distilled TBSOTf ( $84 \mu \mathrm{L}, 1.5$ equiv.). The reaction was then allowed to warm to $-20^{\circ} \mathrm{C}$ over $45 \mathrm{~min}$, and after an additional $2 \mathrm{~h}$, was diluted with sat. $\mathrm{NaHCO}_{3}(3 \mathrm{~mL})$, water $(10 \mathrm{~mL})$ and $\mathrm{CH}_{2} \mathrm{Cl}_{2}(10 \mathrm{~mL})$. The aqueous layer was then washed with $\mathrm{CH}_{2} \mathrm{Cl}_{2}(3 \times 5 \mathrm{~mL})$, and the combined organic layers were washed with $1 \mathrm{M} \mathrm{NaHSO}_{4}(3 \times 5 \mathrm{~mL})$, brine $(10 \mathrm{~mL})$, dried over $\mathrm{MgSO}_{4}$ and concentrated in vacuo to afford $160.0 \mathrm{mg}$ (99\% yield) of (-)-30 as a colorless oil which was used without further purification: $\mathrm{R}_{\mathrm{f}} 0.61$ (4/1 hexanes/EtOAc); $[\alpha]_{D}^{20}-43.0\left(c 0.89, \mathrm{C}_{6} \mathrm{H}_{6}\right)$; IR (neat) 2932 (s), 2857 (s), $1730(\mathrm{~m}), 1613(\mathrm{w}), 1513(\mathrm{~m}), 1465(\mathrm{~m}), 1250(\mathrm{~s}), 1098(\mathrm{~s}) \mathrm{cm}^{-1} ;{ }^{1} \mathrm{H}$ NMR $\left(500 \mathrm{MHz}, \mathrm{CDCl}_{3}\right) \delta 7.25(\mathrm{~d}, \mathrm{~J}=$ $8.6 \mathrm{~Hz}, 2 \mathrm{H}), 6.86(\mathrm{~d}, \mathrm{~J}=8.6 \mathrm{~Hz}, 2 \mathrm{H}), 5.23(\mathrm{~d}, J=9.2 \mathrm{~Hz}, 1 \mathrm{H}), 4.38(\mathrm{br} \mathrm{s}, 2 \mathrm{H}), 4.34(\mathrm{~m}, 1 \mathrm{H}), 4.11(\mathrm{~d}, \mathrm{~J}=$ 8.3 Hz, $1 \mathrm{H}), 3.86(\mathrm{~d}, \mathrm{~J}=8.3 \mathrm{~Hz}, 1 \mathrm{H}), 3.75(\mathrm{~s}, 3 \mathrm{H}), 3.59(\mathrm{~m}, 2 \mathrm{H}), 3.37$ (app t, J=6.7 Hz, $2 \mathrm{H}), 2.67$ (dd, $J=13.8,6.7 \mathrm{~Hz}, 1 \mathrm{H}), 2.36(\mathrm{dd}, J=13.8,3.0 \mathrm{~Hz}, 1 \mathrm{H}), 2.29(\mathrm{~m}, 1 \mathrm{H}), 1.77(\mathrm{~m}, 1 \mathrm{H}), 1.61(\mathrm{~s}, 3 \mathrm{H}), 1.51(\mathrm{~m}$, $4 \mathrm{H}), 1.20(\mathrm{~m}, 5 \mathrm{H}), 0.84(\mathrm{~d}, \mathrm{~J}=6.7 \mathrm{~Hz}, 3 \mathrm{H}), 0.786(\mathrm{~s}, 9 \mathrm{H}), 0.781$ (s, $9 \mathrm{H}), 0.01(\mathrm{~s}, 3 \mathrm{H}),-0.07$ (s, $6 \mathrm{H}),-$ $0.11(\mathrm{~s}, 3 \mathrm{H}) ;{ }^{13} \mathrm{C}$ NMR $\left(125 \mathrm{MHz}, \mathrm{CDCl}_{3}\right) \delta 214.1,163.9,141.5,134.0,133.1,132.2,115.9,83.2,76.5$, 
$72.2,70.1,69.8,58.1,53.9,43.4,34.6,32.5,29.5,26.9,24.2,23.4,22.9,22.7,16.8,14.9,14.8,8.2,-9.0$, $-10.1,-10.2$; high resolution mass spectrum (ES+) $\mathrm{m} / \mathrm{z} 685.4286\left[(\mathrm{M}+\mathrm{Na})^{+}\right.$; calcd for $\mathrm{C}_{37} \mathrm{H}_{66} \mathrm{O}_{6} \mathrm{Si}_{2} \mathrm{Na}^{+}$: 685.4449].

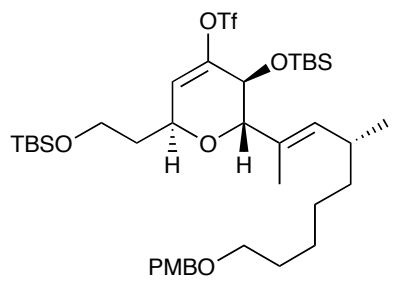

$(-)-\mathrm{SI} 21$

Enol Triflate (-)-SI21. To a $-78{ }^{\circ} \mathrm{C}$ solution of azeotroped ketone (-)-30 (158 mg, 0.238 mmoles) in THF/HMPA (19/1, $8.5 \mathrm{~mL}$ ) was added freshly prepared lithium diisopropyl amide (LDA, $714 \mu \mathrm{L}, 0.5 \mathrm{M}$ in THF, 1.5 equiv.). After $30 \mathrm{~min}$, a solution of azeotroped $N$-(5-chloro-2-pyridyl)triflamide ${ }^{6}$ (140 mg, 1.5 equiv.) in THF/HMPA $(19 / 1,371 \mu \mathrm{L})$ was added dropwise. After $1.5 \mathrm{~h}$, the reaction was quenched with sat. $\mathrm{NH}_{4} \mathrm{Cl}(5 \mathrm{~mL})$, and warmed to ambient temperature. After diluting with water $(15 \mathrm{~mL})$ and diethyl ether $(15 \mathrm{~mL})$, the organic layer was washed with water $(3 \times 10 \mathrm{~mL})$, dried over $\mathrm{MgSO}_{4}$ and concentrated in vacuo to afford the crude enol triflate (-)-SI21 that was used without further purification: $R_{\mathrm{f}} 0.38(10 / 1$ hexanes/EtOAc); $[\alpha]_{\mathrm{D}}^{20}-35.2\left(c 0.42, \mathrm{C}_{6} \mathrm{H}_{6}\right) ; \mathrm{IR}$ (thin film, $\left.\mathrm{CDCl}_{3}\right) 2930(\mathrm{~s}), 2857(\mathrm{~s}), 1614(\mathrm{w}), 1513(\mathrm{~m})$, $1462(\mathrm{~m}), 1421(\mathrm{~m}), 1362$ (w), 1301 (w), 1248 (s), 1212 (s), 1143 (s), 1097 (s), 1039 (m) cm ${ }^{-1} ;{ }^{1} \mathrm{H}$ NMR $\left(500 \mathrm{MHz}, \mathrm{CDCl}_{3}\right) \delta 7.25(\mathrm{~d}, J=8.6 \mathrm{~Hz}, 2 \mathrm{H}), 6.87(\mathrm{~d}, J=8.6 \mathrm{~Hz}, 2 \mathrm{H}), 5.89(\mathrm{~d}, J=2.1 \mathrm{~Hz}, 1 \mathrm{H}), 5.17(\mathrm{~d}, J=$ $9.2 \mathrm{~Hz}, 1 \mathrm{H}), 4.41(\mathrm{br} \mathrm{s}, 2 \mathrm{H}), 4.37(\mathrm{br} \mathrm{s}, 1 \mathrm{H}), 4.23(\mathrm{~m}, 1 \mathrm{H}), 4.08(\mathrm{~d}, \mathrm{~J}=2.7 \mathrm{~Hz}, 1 \mathrm{H}), 3.80(\mathrm{~s}, 3 \mathrm{H}), 3.73$ (m, 2 H), $3.41($ app t, J= $6.7 \mathrm{~Hz}, 2 \mathrm{H}), 2.36(\mathrm{~m}, 1 \mathrm{H}), 1.85(\mathrm{~m}, 1 \mathrm{H}), 1.80(\mathrm{~m}, 1 \mathrm{H}), 1.67(\mathrm{~s}, 3 \mathrm{H}), 1.58(\mathrm{~m}, 2$ H), 1.33-1.16 (m, $6 \mathrm{H}), 0.92(\mathrm{~d}, J=6.8 \mathrm{~Hz}, 3 \mathrm{H}), 0.89(\mathrm{~s}, 9 \mathrm{H}), 0.87(\mathrm{~s}, 9 \mathrm{H}), 0.12(\mathrm{~s}, 3 \mathrm{H}), 0.06(\mathrm{~s}, 3 \mathrm{H})$, 0.03 (s, $6 \mathrm{H}) ;{ }^{13} \mathrm{C}$ NMR $\left(125 \mathrm{MHz}, \mathrm{CDCl}_{3}\right) \delta 159.0,146.4,136.4,130.7,129.1,128.6,122.9,113.7,82.4$, $72.4,70.1,66.3,66.0,59.0,55.2,36.9,36.5,32.4,29.7,27.3,26.3,25.8,25.7,20.3,19.4,18.2,18.0$, $14.1,-4.6,-5.4$; high resolution mass spectrum $(E S+) \mathrm{m} / \mathrm{z} 817.3773 \quad\left[(\mathrm{M}+\mathrm{Na})^{+}\right.$; calcd for $\mathrm{C}_{38} \mathrm{H}_{65} \mathrm{~F}_{3} \mathrm{O}_{8} \mathrm{SSi}_{2} \mathrm{Na}^{+}:$571.3533]. 


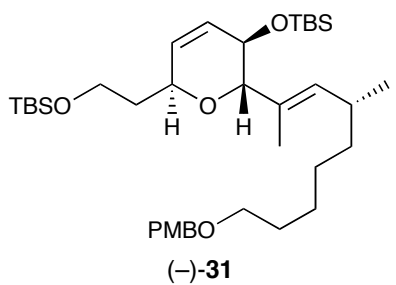

Diene (-)-31. To flame-dried $\mathrm{LiCl}(21.6 \mathrm{mg}, 3.0$ equiv.) was added a solution of the crude enol triflate (-)-SI21 (135 mg, 0.170 mmoles) in THF (3.5 mL). A Pd $(\mathrm{dba})_{3} \cdot \mathrm{CHCl}_{3} / \mathrm{PPh}_{3} / \mathrm{THF}$ solution was then added dropwise $\left(200 \mu \mathrm{L}, 0.025\right.$ equiv., prepared with $42.5 \mu$ moles $\mathrm{Pd}_{2}\left(\mathrm{dba}_{3} \cdot \mathrm{CHCl}_{3}\right.$, and $341 \mu \mathrm{moles}$ $\mathrm{PPh}_{3}$ in $2 \mathrm{~mL} \mathrm{THF}$ ) and the solution heated to reflux. $\mathrm{Bu}_{3} \mathrm{SnH}(46 \mu \mathrm{L}, 1.0$ equiv.) was then added dropwise. Gas Evolution! After $1 \mathrm{~h}$, and additional $200 \mu \mathrm{L}$ (0.025 equiv.) of the $\mathrm{Pd}_{2}(\mathrm{dba})_{3} \cdot \mathrm{CHCl}_{3} / \mathrm{PPh}_{3} /$ THF solution and $\mathrm{Bu}_{3} \mathrm{SnH}$ (69 $\mu \mathrm{L}, 1.5$ equiv.) were sequentially added dropwise. After an additional $1 \mathrm{~h}$, the reaction was cooled to ambient temperature and diluted with water $(10 \mathrm{~mL})$ and diethyl ether $(10 \mathrm{~mL})$. The organic layer was then dried over $\mathrm{MgSO}_{4}$ and concentrated in vacuo. Purification by flash chromatography (20/1, hexanes/EtOAc) afforded $70.3 \mathrm{mg}(71 \%$ yield, 2 steps) of (-)-31 as a pale yellow oil: $\mathrm{R}_{\mathrm{f}} 0.38$ (10/1 hexanes/EtOAc); $[\alpha]_{\mathrm{D}}^{20}-74.5\left(c 0.17, \mathrm{C}_{6} \mathrm{H}_{6}\right)$; IR (thin film, $\left.\mathrm{CDCl}_{3}\right) 2929$ (s), 2856 (s), $1720(w), 1613(w), 1512(m), 1476(m), 1360(w), 1300(w), 1249(s), 1172(w), 1099(b r, s), 1038(w)$ $\mathrm{cm}^{-1} ;{ }^{1} \mathrm{H}$ NMR $\left(500 \mathrm{MHz}, \mathrm{CDCl}_{3}\right) \delta 7.26(\mathrm{~d}, \mathrm{~J}=8.6 \mathrm{~Hz}, 2 \mathrm{H}), 6.87(\mathrm{~d}, \mathrm{~J}=8.6 \mathrm{~Hz}, 2 \mathrm{H}), 5.71$ (br s, $\left.2 \mathrm{H}\right), 5.25$ $(\mathrm{d}, J=9.1 \mathrm{~Hz}, 1 \mathrm{H}), 4.43(\mathrm{br} \mathrm{s}, 2 \mathrm{H}), 4.30(\mathrm{dd}, J=9.4,3.8 \mathrm{~Hz}, 1 \mathrm{H}), 4.16(\mathrm{dd}, J=7.7,1.6 \mathrm{~Hz}, 1 \mathrm{H}), 3.80(\mathrm{~s}$, $3 \mathrm{H}) ; 3.71(\mathrm{~m}, 2 \mathrm{H}), 3.62(\mathrm{~d}, J=7.7 \mathrm{~Hz}, 1 \mathrm{H}), 3.42(\operatorname{app~t}, J=6.6 \mathrm{~Hz}, 2 \mathrm{H}), 2.37(\mathrm{~m}, 1 \mathrm{H}), 1.89(\mathrm{~m}, 1 \mathrm{H})$, $1.69(\mathrm{~m}, 1 \mathrm{H}), 1.64(\mathrm{~s}, 3 \mathrm{H}), 1.59(\mathrm{~m}, 2 \mathrm{H}), 1.35-1.20(\mathrm{~m}, 6 \mathrm{H}), 0.93(\mathrm{~d}, \mathrm{~J}=6.7 \mathrm{~Hz}, 3 \mathrm{H}), 0.88(\mathrm{~s}, 9 \mathrm{H}), 0.87$ (s, $9 \mathrm{H}), 0.06(\mathrm{~s}, 3 \mathrm{H}), 0.04(\mathrm{~s}, 6 \mathrm{H}), 0.02(\mathrm{~s}, 3 \mathrm{H}) ;{ }^{13} \mathrm{C}$ NMR $\left(125 \mathrm{MHz}, \mathrm{CDCl}_{3}\right) \delta$ 159.0, 136.4, 131.1, $130.8,130.3,130.0,129.1,113.7,78.6,72.4,70.2,69.7,66.0,60.2,55.2,37.2,35.7,32.0,29.7,27.2$ $26.4,25.9,25.8,20.4,18.3,18.1,12.8,-4.2,-4.5,-5.3$; high resolution mass spectrum (ES+) $\mathrm{m} / \mathrm{z}$ $669.4372\left[(\mathrm{M}+\mathrm{Na})^{+}\right.$; calcd for $\left.\mathrm{C}_{37} \mathrm{H}_{66} \mathrm{O}_{5} \mathrm{Si}_{2} \mathrm{Na}^{+}: 669.4346\right]$.

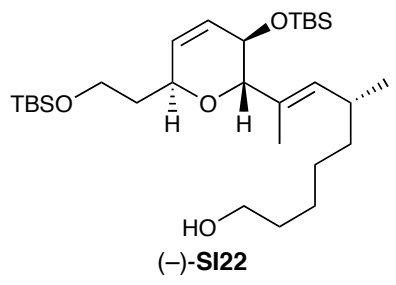


Alcohol (-)-SI22. To a $0{ }^{\circ} \mathrm{C}$ solution of PMB ether (-)-31 (40.0 mg, $61.8 \mu$ moles) in $\mathrm{CH}_{2} \mathrm{Cl}_{2}(2$ $\mathrm{mL})$ and $\mathrm{pH} 7$ buffer (100 $\mu \mathrm{L})$ was added DDQ (21.0 mg, 1.5 equiv.) to produce a blue/green suspension. After $40 \mathrm{~min}$, additional DDQ (14.0 mg, 1.0 equiv.) was added, and after $30 \mathrm{~min}$, the suspension was diluted with sat. $\mathrm{NaHCO}_{3}(3 \mathrm{~mL})$, water $(10 \mathrm{~mL})$ and $\mathrm{CH}_{2} \mathrm{Cl}_{2}(15 \mathrm{~mL})$. The aqueous layer was then washed with $\mathrm{CH}_{2} \mathrm{Cl}_{2}(3 \times 5 \mathrm{~mL})$, and the combined organic layers were dried over $\mathrm{MgSO}_{4}$ and concentrated in vacuo. Purification by flash chromatography (10/1, hexanes/EtOAc) afforded $33.6 \mathrm{mg}$ of a mixture of (-)-SI22 and p-anisaldehyde which was used without further purification. Further flash chromatography afforded clean (-)-SI22 for characterization purposes: $R_{f} 0.13$ (10/1 hexanes/EtOAc); $[\alpha]_{\mathrm{D}}^{20}-36.6\left(c\right.$ 0.62, $\mathrm{CDCl}_{3}$ ); IR (thin film, $\mathrm{CDCl}_{3}$ ) 3363 (br, w), $2929(\mathrm{~s}), 2857(\mathrm{~s}), 1471(\mathrm{~m}), 1388(\mathrm{w})$, 1361 (w), 1300 (w), 1254 (s), 1100 (br, s) cm ${ }^{-1}$; ${ }^{1} \mathrm{H}$ NMR (500 MHz, $\mathrm{CDCl}_{3}$ ) $\delta 5.71$ (br s, $2 \mathrm{H}$ ), 5.25 (dd, J= 9.2, $1.1 \mathrm{~Hz}, 1 \mathrm{H}), 4.30(\mathrm{~m}, 1 \mathrm{H}), 4.16(\mathrm{dd}, \mathrm{J}=7.8,2.0 \mathrm{~Hz}, 1 \mathrm{H}), 3.72(\mathrm{~m}, 2 \mathrm{H}), 3.63(\operatorname{app~t}, J=6.6 \mathrm{~Hz}, 2 \mathrm{H})$, $3.62(\mathrm{~d}, J=7.8 \mathrm{~Hz}, 1 \mathrm{H}), 2.38(\mathrm{~m}, 1 \mathrm{H}), 1.88(\mathrm{~m}, 1 \mathrm{H}), 1.68(\mathrm{~m}, 1 \mathrm{H}), 1.64(\mathrm{~d}, J=1.3 \mathrm{~Hz}, 3 \mathrm{H}), 1.55(\mathrm{~m}, 2$ H), $1.30(\mathrm{~m}, 6 \mathrm{H}), 0.94(\mathrm{~d}, \mathrm{~J}=6.6 \mathrm{~Hz}, 3 \mathrm{H}), 0.88(\mathrm{~s}, 9 \mathrm{H}), 0.87$ (s, $9 \mathrm{H}), 0.06(\mathrm{~s}, 3 \mathrm{H}), 0.04(\mathrm{~s}, 6 \mathrm{H}), 0.02(\mathrm{~s}$, $3 \mathrm{H}) ;{ }^{13} \mathrm{C} \mathrm{NMR}\left(125 \mathrm{MHz}, \mathrm{CDCl}_{3}\right) \delta 136.3,131.2,130.3,129.9,78.5,69.7,66.1,63.0,60.2,37.2,35.7$, $32.7,32.0,27.1,25.9(2), 25.8,20.4,18.3,18.0,12.8,-4.2,-4.5,-5.3$; high resolution mass spectrum (ES+) m/z 549.3756 [(M+Na) ${ }^{+}$; calcd for $\mathrm{C}_{29} \mathrm{H}_{58} \mathrm{O}_{4} \mathrm{Si}_{2} \mathrm{Na}^{+}:$549.3874] .

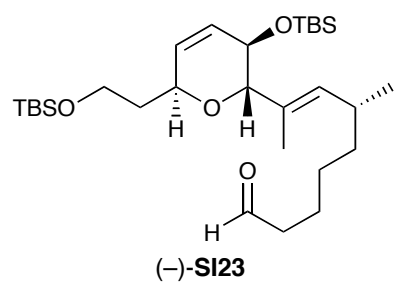

Aldehyde (-)-SI23. To a $0{ }^{\circ} \mathrm{C}$ solution of the above alcohol (-)-SI22 mixture $(32.5 \mathrm{mg}, 61.8$ umoles) and pyridine (23 $\mu \mathrm{L}, 5.0$ equiv.) in $\mathrm{CH}_{2} \mathrm{Cl}_{2}(3 \mathrm{~mL})$ was added Dess-Martin periodinane (49.2 mg, 2.0 equiv.). The white suspension was then warmed to ambient temperature, and after $1 \mathrm{~h}$, was diluted with sat. $\mathrm{NaHCO}_{3}(3 \mathrm{~mL})$, water $(10 \mathrm{~mL})$, and $\mathrm{CH}_{2} \mathrm{Cl}_{2}(15 \mathrm{~mL})$. The aqueous layer was then washed with $\mathrm{CH}_{2} \mathrm{Cl}_{2}(3 \times 5 \mathrm{~mL})$, and the combined organic layers were dried over $\mathrm{Na}_{2} \mathrm{SO}_{4}$ and concentrated in vacuo. Purification by flash chromatography (20/1, hexanes/EtOAc) afforded $27.8 \mathrm{mg}$ ( $86 \%$ yield, 2 steps) of (-)SI23 as a pale yellow oil: $\mathrm{R}_{\mathrm{f}} 0.26$ (20/1 hexanes/EtOAc); $[\alpha]_{\mathrm{D}}^{20}-39.2\left(c 1.66, \mathrm{CDCl}_{3}\right)$; IR (thin film, $\left.\mathrm{CDCl}_{3}\right)$ 
2954 (s), 2856 (s), 2711 (w), 1728 (s), 1462 (s), $1388(\mathrm{~m}), 1361$ (m), 1252 (s), 1098 (br, s) cm ${ }^{-1}$; ${ }^{1} \mathrm{H}$ NMR $\left(500 \mathrm{MHz}, \mathrm{CDCl}_{3}\right) \delta 9.75(\operatorname{app~t}, J=1.9 \mathrm{~Hz}, 1 \mathrm{H}), 5.71(\mathrm{br} \mathrm{s}, 2 \mathrm{H}), 5.24(\mathrm{~d}, J=9.1 \mathrm{~Hz}, 1 \mathrm{H}), 4.30(\mathrm{dd}, J=$ 9.3, 4.1 Hz, $1 \mathrm{H}$ ), $4.15(\mathrm{dd}, J=7.8,2.2 \mathrm{~Hz}, 1 \mathrm{H}), 3.72(\mathrm{~m}, 2 \mathrm{H}), 3.61(\mathrm{~d}, J=7.8 \mathrm{~Hz}, 1 \mathrm{H}), 2.40$ (app dt, J= 7.8, $1.9 \mathrm{~Hz}, 2 \mathrm{H}), 2.39(\mathrm{~m}, 1 \mathrm{H}), 1.88(\mathrm{~m}, 1 \mathrm{H}), 1.69(\mathrm{~m}, 1 \mathrm{H}), 1.64(\mathrm{~s}, 3 \mathrm{H}), 1.60(\mathrm{~m}, 2 \mathrm{H}), 1.38-1.20(\mathrm{~m}, 4$ H), $0.94(\mathrm{~d}, \mathrm{~J}=6.7 \mathrm{~Hz}, 3 \mathrm{H}), 0.88(\mathrm{~s}, 9 \mathrm{H}), 0.87(\mathrm{~s}, 9 \mathrm{H}), 0.06(\mathrm{~s}, 3 \mathrm{H}), 0.04(\mathrm{~s}, 6 \mathrm{H}), 0.02(\mathrm{~s}, 3 \mathrm{H}) ;{ }^{13} \mathrm{C} \mathrm{NMR}$ $\left(125 \mathrm{MHz}, \mathrm{CDCl}_{3}\right) \delta$ 202.5, 135.8, 131.5, 130.3, 129.9, 78.4, 69.7, 66.1, 60.1, 43.8, 36.9, 35.7, 31.8, 29.6, $26.9,25.9,25.8,20.3,18.2,18.0,12.8,-4.2,-4.5,-5.4$; high resolution mass spectrum (ES+) $\mathrm{m} / \mathrm{z}$ 547.3612 [(M+Na) $)^{+}$; calcd for $\mathrm{C}_{29} \mathrm{H}_{56} \mathrm{O}_{4} \mathrm{Si}_{2} \mathrm{Na}^{+}:$524.3717].

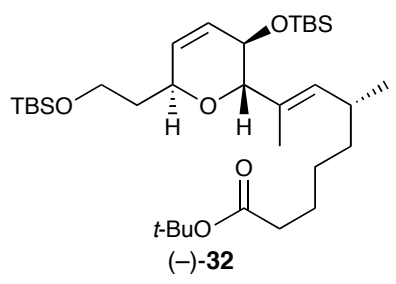

Ester (-)-32. To a solution of aldehyde (-)-SI23 (33.2 mg, $63.2 \mu$ moles) in tert-butanol (1.2 mL) was added 2-methyl-2-butene $(\sim 500 \mu \mathrm{L})$, followed by a solution of sodium chlorite $(35.7 \mathrm{mg}, 80 \%, 5.0$ equiv.) and sodium dihydrogenphosphate $(75.8 \mathrm{mg}, 10.0$ equiv.) in water (360 $\mu \mathrm{L})$. After $1.5 \mathrm{~h}$, the reaction was diluted with sat. $\mathrm{NH}_{4} \mathrm{Cl}(10 \mathrm{~mL})$ and EtOAc $(10 \mathrm{~mL})$. The aqueous layer was then washed with EtOAc $(4 \times 5 \mathrm{~mL})$, and the combined organic layers were dried over $\mathrm{MgSO}_{4}$ and concentrated in vacuo to afford the crude carboxylic acid that was used without further purification. To a solution of the crude carboxylic acid $(34.1 \mathrm{mg}, 63.0 \mu$ moles $)$ in dichloromethane $(4 \mathrm{~mL})$ was added $N, N$ '-diisopropyl- $O$ tert-butylisourea ${ }^{7}(45 \mu \mathrm{L}, 3.0$ equiv.). After $24 \mathrm{~h}$, and $48 \mathrm{~h}$, an additional 3.0 equiv. of the isourea reagent were added, and after an additional $12 \mathrm{~h}$, the now white suspension was filtered through celite and concentrated in vacuo. Purification by flash chromatography $(20 / 1 \rightarrow 10 / 1$, hexanes/diethyl ether) afforded $29.9 \mathrm{mg}$ (80\% yield, 2 steps) of (-)-32 as a pale yellow oil: $\mathrm{R}_{\mathrm{f}} 0.17$ (10/1 hexanes/diethyl ether); $[\alpha]_{\mathrm{D}}^{20}-24.8\left(c\right.$ 1.49, $\mathrm{CDCl}_{3}$ ); IR (thin film, $\mathrm{CH}_{2} \mathrm{Cl}_{2}$ ) 3031 (w), $2929(\mathrm{~s}), 2857$ (s), $1732(\mathrm{~s}), 1604(\mathrm{w}), 1471$ (m), $1462(\mathrm{~m}), 1388(\mathrm{~m}), 1366(\mathrm{~m}), 1252(\mathrm{~s}), 1134(\mathrm{~s}), 1099(\mathrm{~s}) \mathrm{cm}^{-1} ;{ }^{1} \mathrm{H}$ NMR $\left(500 \mathrm{MHz}, \mathrm{CDCl}_{3}\right) \delta 5.70$ (br s, $2 \mathrm{H}), 5.24(\mathrm{dd}, J=9.2,1.0 \mathrm{~Hz}, 1 \mathrm{H}), 4.29(\mathrm{dd}, J=9.5,4.3 \mathrm{~Hz}, 1 \mathrm{H}), 4.15(\mathrm{dd}, J=7.8,2.1 \mathrm{~Hz}, 1 \mathrm{H})$, $3.71(\mathrm{~m}, 2 \mathrm{H}), 3.61(\mathrm{~d}, J=7.8 \mathrm{~Hz}, 1 \mathrm{H}), 2.37(\mathrm{~m}, 1 \mathrm{H}), 2.18(\operatorname{app~t}, J=7.6 \mathrm{~Hz}, 2 \mathrm{H}), 1.88(\mathrm{~m}, 1 \mathrm{H}), 1.68(\mathrm{~m}$, 
$1 \mathrm{H}), 1.64(\mathrm{~s}, 3 \mathrm{H}), 1.55(\mathrm{~m}, 2 \mathrm{H}), 1.43(\mathrm{~s}, 9 \mathrm{H}), 1.30-1.20(\mathrm{~m}, 4 \mathrm{H}), 0.93(\mathrm{~d}, \mathrm{~J}=6.7 \mathrm{~Hz}, 3 \mathrm{H}), 0.88(\mathrm{~s}, 9 \mathrm{H})$, 0.87 (s, $9 \mathrm{H}), 0.06$ (s, $3 \mathrm{H}), 0.03$ (s, $6 \mathrm{H}), 0.01$ (s, $3 \mathrm{H}) ;{ }^{13} \mathrm{C}$ NMR $\left(125 \mathrm{MHz}, \mathrm{CDCl}_{3}\right) \delta$ 173.2, 136.1, 131.3, $130.3,130.0,79.8,78.5,69.7,66.1,60.1,36.8,35.5,31.9,29.7,28.1,26.8,25.9,25.8,25.2,20.3,18.3$, 18.0, 12.8, -4.2, -4.5, -5.3; high resolution mass spectrum $(E S+) \mathrm{m} / \mathrm{z} 619.4213\left[(\mathrm{M}+\mathrm{Na})^{+}\right.$; calcd for $\mathrm{C}_{33} \mathrm{H}_{64} \mathrm{O}_{5} \mathrm{Si}_{2} \mathrm{Na}^{+}:$619.4292].

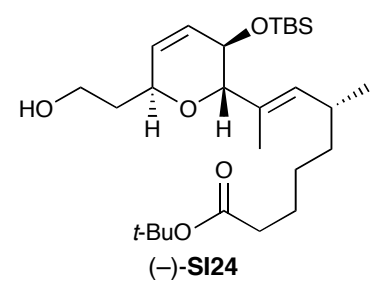

Alcohol (-)-SI24. To a solution of TBS-ether (-)-32 (7.5 mg, $12.5 \mu$ moles) and THF (500 $\mu \mathrm{L})$ in a Nalgene container was added a stock solution of $\mathrm{HF}^{*}$ pyridine $(76 \mu \mathrm{L}$, prepared by adding $6.35 \mathrm{~mL}$ pyridine portionwise to a solution of $2.59 \mathrm{~g} \mathrm{HF}^{*}$ pyridine and $20 \mathrm{~mL}$ THF in a $30 \mathrm{~mL}$ Nalgene container). After $31 \mathrm{~h}$, the reaction was diluted with sat. $\mathrm{NH}_{4} \mathrm{Cl}(10 \mathrm{~mL})$ and $\mathrm{EtOAc}(15 \mathrm{~mL})$. The aqueous layer was then washed with EtOAc $(3 \times 5 \mathrm{~mL})$, and the combined organic layers were dried over $\mathrm{MgSO}_{4}$ and concentrated in vacuo. Purification by flash chromatography (10/1 $\rightarrow 4 / 1$, hexanes/EtOAc) afforded 4.5 $\mathrm{mg}\left(75 \%\right.$ yield, $\sim 88 \%$ conversion) of $(-)-\mathrm{SI} 24$ as a pale yellow oil: $\mathrm{R}_{\mathrm{f}} 0.20\left(10 / 1\right.$ hexanes/EtOAc); $[\alpha]_{D}^{20}-$ 43.2 (c 0.55, $\mathrm{CDCl}_{3}$ ); IR (thin film, $\mathrm{CH}_{2} \mathrm{Cl}_{2}$ ) 3446 (br, m), 3036 (w), 2930 (s), 2857 (s), 1731 (s), 1458 (m), 1389 (m), 1367 (m), 1252 (s), 1154 (s), 1122 (s), 1093 (s), 1068 (s) cm ${ }^{-1} ;{ }^{1} \mathrm{H}$ NMR (500 MHz, CDCl ${ }_{3}$ ) $5.76(\mathrm{dd}, J=10.4,0.8 \mathrm{~Hz}, 1 \mathrm{H}), 5.66$, (ddd, J= 10.4, 1.2, $1.2 \mathrm{~Hz}, 1 \mathrm{H}), 5.29$ (d, J= 9.0 Hz, $1 \mathrm{H}), 4.34$ (br d, J= 10.3 Hz, $1 \mathrm{H}), 4.17(\mathrm{~d}, J=7.4 \mathrm{~Hz}, 1 \mathrm{H}), 3.79(\mathrm{~m}, 2 \mathrm{H}), 3.75(\mathrm{~d}, J=7.4 \mathrm{~Hz}, 1 \mathrm{H}), 2.72(\mathrm{dd}, J=8.1,3.2 \mathrm{~Hz}$, $1 \mathrm{H}), 2.37(\mathrm{~m}, 1 \mathrm{H}), 2.18(\operatorname{app~t}, J=7.4 \mathrm{~Hz}, 2 \mathrm{H}), 2.02(\mathrm{~m}, 1 \mathrm{H}), 1.65(\mathrm{~s}, 3 \mathrm{H}), 1.63(\mathrm{~m}, 1 \mathrm{H}), 1.55(\mathrm{~m}, 2 \mathrm{H})$, $1.44(\mathrm{~s}, 9 \mathrm{H}), 1.26(\mathrm{~m}, 4 \mathrm{H}), 0.93(\mathrm{~d}, \mathrm{~J}=6.7 \mathrm{~Hz}, 3 \mathrm{H}), 0.87(\mathrm{~s}, 9 \mathrm{H}), 0.07(\mathrm{~s}, 3 \mathrm{H}), 0.03(\mathrm{~s}, 3 \mathrm{H}) ;{ }^{13} \mathrm{C}$ NMR $\left(125 \mathrm{MHz}, \mathrm{CDCl}_{3}\right) \delta 173.2,137.1,130.7,130.1,129.6,79.8,78.7,73.4,65.6,61.9,36.7,35.5,34.3,31.9$, $28.1,26.8,25.8,25.2,20.3,18.0,12.9,-4.2,-4.6$; high resolution mass spectrum (ES+) m/z 505.3490 $\left[(\mathrm{M}+\mathrm{Na})^{+}\right.$; calcd for $\mathrm{C}_{27} \mathrm{H}_{50} \mathrm{O}_{5} \mathrm{SiNa}^{+}:$505.3428]. 


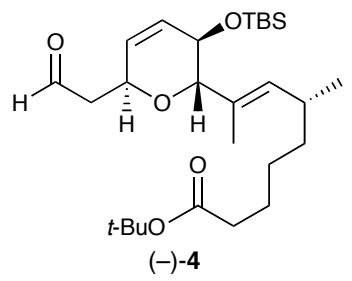

Aldehyde (-)-4. To a solution of alcohol (-)-SI24 (4.5 mg, $9.3 \mu$ moles) and pyridine $(4 \mu \mathrm{L}, 5.0$ equiv.) in $\mathrm{CH}_{2} \mathrm{Cl}_{2}(600 \mu \mathrm{L})$ was added Dess-Martin periodinane $(7.9 \mathrm{mg}, 2.0$ equiv.). After $1 \mathrm{~h}$, the white suspension was diluted with sat. $\mathrm{NaHCO}_{3}(3 \mathrm{~mL})$, water $(10 \mathrm{~mL})$ and $\mathrm{CH}_{2} \mathrm{Cl}_{2}(15 \mathrm{~mL})$. The aqueous layer was then washed with $\mathrm{CH}_{2} \mathrm{Cl}_{2}(3 \times 5 \mathrm{~mL})$, and the combined organic layers were dried over $\mathrm{MgSO}_{4}$ and concentrated in vacuo. Purification by flash chromatography (10/1, hexanes/EtOAc) afforded $3.8 \mathrm{mg}$ (86\% yield) of (-)-4 as a pale yellow oil: $\mathrm{R}_{\mathrm{f}} 0.11\left(10 / 1\right.$ hexanes/EtOAc); $[\alpha]_{\mathrm{D}}^{20}-37.8\left(c 0.19, \mathrm{C}_{6} \mathrm{D}_{6}\right)$; IR (thin film, $\left.\mathrm{C}_{6} \mathrm{H}_{6}\right) 2931$ (br, s), 2857 (m), 1727 (br, s), 1461 (w), 1369 (w), 1253 (w), $1153(\mathrm{~m}), 1091$ (m) cm ${ }^{-1} ;{ }^{1} \mathrm{H}$ NMR (500 MHz, $\left.\mathrm{C}_{6} \mathrm{D}_{6}\right) \delta 9.32(\mathrm{br} \mathrm{s}, 1 \mathrm{H}), 5.73$ (ddd, J=10.3, 2.0, $2.0 \mathrm{~Hz}, 1 \mathrm{H}$ ), 5.39 (ddd, J= 10.3, 2.0, 2.0 $\mathrm{Hz}, 1 \mathrm{H}), 5.36(\mathrm{~d}, J=9.5 \mathrm{~Hz}, 1 \mathrm{H}), 4.49(\mathrm{~m}, 1 \mathrm{H}), 4.20(\mathrm{dd}, J=7.5,2.0 \mathrm{~Hz}, 1 \mathrm{H}), 3.75(\mathrm{~d}, J=7.5 \mathrm{~Hz}, 1 \mathrm{H})$, 2.36 (ddd, J=16.4, 8.4, $2.5 \mathrm{~Hz}, 1 \mathrm{H}$ ), 2.32 (m, 1 H), 2.17 (app t, J= $7.4 \mathrm{~Hz}, 2 \mathrm{H}$ ), 1.88 (ddd, J= 16.4, 5.4, 1.2, $5.4 \mathrm{~Hz}, 1 \mathrm{H}), 1.71(\mathrm{~s}, 3 \mathrm{H}), 1.61(\mathrm{~m}, 2 \mathrm{H}), 1.39(\mathrm{~s}, 9 \mathrm{H}), 1.26(\mathrm{~m}, 4 \mathrm{H}), 0.95(\mathrm{~s}, 9 \mathrm{H}), 0.91(\mathrm{~d}, J=6.7$ $\mathrm{Hz}, 3 \mathrm{H}), 0.03(\mathrm{~s}, 3 \mathrm{H}), 0.02(\mathrm{~s}, 3 \mathrm{H}) ;{ }^{13} \mathrm{C}$ NMR (125 MHz, $\left.\mathrm{C}_{6} \mathrm{D}_{6}\right) \delta$ 197.7, 171.4, 135.5, 130.5, 130.1, 128.0, 78.3, 78.2, 67.3, 65.0, 45.9, 36.2, 34.6, 31.2, 27.1, 26.1, 25.0, 24.6, 19.5, 17.1, 12.2, -5.1, -5.4; high resolution mass spectrum (ES+) $\mathrm{m} / \mathrm{z} 503.3157\left[(\mathrm{M}+\mathrm{Na})^{+}\right.$; calcd for $\left.\mathrm{C}_{27} \mathrm{H}_{48} \mathrm{O}_{5} \mathrm{SiNa}^{+}: 503.3271\right]$.

${ }^{1}$ Burchat, A. F.; Chong, J. M.; Nielsen, N. J. Organometallic Chem. 1997, 542, 281.

${ }^{2}$ Smith, A. B., III; Minbiole, K. P.; Verhoest, P. R.; Schelhaas, M. J. Am. Chem. Soc. 2001, 123, 10942.

${ }^{3}$ Nicolau, K. C.; Papahatjis, D. P.; Claremon, D. A.; Magolda, R. L.; Dolle, R. E. J. Org. Chem. 1985, 50, 1440.

${ }^{4}$ Kubota, T.; Tsuda, M.; Kobayashi, J. J. Org. Chem. 2002, 67, 1651.

${ }^{5}$ Keck, G. E.; Li, X.-Y.; Krishnamurthy, D. J. Org. Chem. 1995, 60, 5998.

${ }^{6}$ Comins, D. L.; Dehghani, A. Tetrahedron Lett. 1992, 33, 6299.

${ }^{7}$ For the preparation and use of $N, N$ '-diisopropyl-O-tert-butylisourea, see Mathias, L. J. Synthesis, 1979, 561. 